\title{
Evaluating Adversarial Learning Resilience on Various Encrypted Traffic Classifications
}

by

\section{Ramy Maarouf}

A thesis submitted to the Faculty of Graduate and Postdoctoral Affairs in partial fulfillment of the requirements for the degree of

\author{
Master of Applied Science \\ in \\ Information Technology
}

\author{
Carleton University \\ Ottawa, Ontario
}

August, 2021

(C) 2021

Ramy Maarouf 


\section{Abstract}

Machine learning and deep learning algorithms are applied to reduce false alarm rates, improve accuracy in classifying encrypted network applications, and improve resilience against multiple types of network attacks. Due to the fast growth of learning techniques, various types of adversarial attacks are increased to reveal ML and DL vulnerabilities and add more challenges in classifying encrypted traffic. In this research, we focus on investigating and evaluating the effectiveness of different adversarial attacks and see how resilient ML and DL algorithms are in classifying encrypted traffic applications, i.e., C4.5 Decision Tree, K-Nearest Neighbor (KNN), Artificial Neural Network (ANN), Convolutional Neural Networks (CNN) and Recurrent Neural Networks (RNN). We train our models in the adversarial-free environment on ISCX VPN-NonVPN and NIMS encrypted traffic datasets using the same top features generated from the feature selection method. Then, we use adversarial samples to attack the trained models and check their resilience in classifying the encrypted applications. Finally, we repeat the experiments and retrain the models using the top nine features per each dataset in the adversarial-free and adversarial environment to compare the effect of the adversarial samples in both scenarios. With our experiments on multiple learning algorithms and two benchmark datasets, we demonstrate that DL shows better resilience against the adversarial samples in encrypted traffic classifications in comparison to ML. We also indicate that the ML and DL model's performance in 
an adversarial-free environment and their resilience against adversarial samples was improved when using more top selected features. However, the impact of the attack varies depending on the type of attack. 
To my Parents: Mohamed and Amira

To my Lovely Wife: Fatma

To my Mother-in-law: Hana

To my Kids: Layla and Ramsey 


\section{Acknowledgments}

I feel immense pleasure to extend my sincere gratitude to my supervisor, Professor Ashraf Matrawy, the lead of the Next Generation Networks (NGN) research group at Carleton University for providing me with valuable support, guidance and encouragement through this research. It was a great privileged and honor to work and study under his guidance that assisted me in extending my knowledge and professional experience while directing me on the right path to pursue my goal. I am expanding my sincere to our NGN Research Group Postdoctoral: Dr. Danish Sattar for his support, advice, and help throughout my study.

I'm very thankful to my beloved wife and soulmate Fatma Arafah for her unconditional love, support, patience, and encouragement during tough times, for offering me the push and positive energy to continue when I am wretched, and for making me believe in myself. A big thanks to my kids, Layla and Ramsey, for their endless love and smile that boosts my power every time I play with them.

My special thanks to my parents: Mohamed Maarouf and Amira Essawy. I owe you everything in my life; your countless scarifies made me the man I am now. Your love, prayer, and belief in me have made it possible to reach for my dreams. I hope I always make you as proud as you make me. My brothers, Sherif and Hazem, thank you for all the love and support you had shown through my study and my whole life; you both are my backbones. 
A particular appreciation goes to my dearest mother-in-law Hana Sany for her true love and limitless prayers in good and bad times. Thanks for the motivation and support each call and for never letting me lose hope. 


\section{Table of Contents}

$\begin{array}{ll}\text { Abstract } & \text { ii }\end{array}$

Acknowledgments $\quad$ v

Table of Contents vii

List of Tables $\quad$ xi

List of Figures $\quad$ xiv

List of Abbreviations $\quad$ xviii

1 Introduction 1

1.1 Research Questions . . . . . . . . . . . . . . . . . . . 2

1.2 Thesis Contributions . . . . . . . . . . . . . . . 3

1.3 Research Papers . . . . . . . . . . . . . . . . . . . . 4

1.4 Thesis Organization . . . . . . . . . . . . . . . . . 5

$\begin{array}{lll}2 & \text { Background } & 7\end{array}$

2.1 Network Security . . . . . . . . . . . . . . . . . 7

2.2 Machine Learning Models . . . . . . . . . . . . . . . . . 8

2.2 .1 Decision Tree $(\mathrm{C} 4.5) \ldots \ldots \ldots \ldots$

2.2 .2 K-Nearest Neighbor $(\mathrm{KNN}) \ldots \ldots \ldots \ldots$ 
2.3 Deep Learning Models . . . . . . . . . . . . . . . . . . . . . . . . . 10

2.3.1 Artificial Neural Network (ANN) _ . . . . . . . . . . . 11

2.3.2 Convolutional Neural Networks (CNN) . . . . . . . . . . 11

2.3.3 Recurrent Neural Networks (RNN) and LSTM . . . . . . . 13

2.4 Adversarial Attack Classification and Threat Model . . . . . . . . . 15

2.4.1 Adversarial Samples . . . . . . . . . . . . . . . . . . 15

2.4.2 Adversarial Threat Model . . . . . . . . . . . . . . . . . 16

2.4.3 Adversarial Samples Generation . . . . . . . . . . . . . . 18

2.5 Literature Review of Traffic Classification using ML . . . . . . . . 20

2.6 Literature Review of Traffic Classification using DL . . . . . . . . 22

2.7 Literature Review of Adversarial Attacks . . . . . . . . . . . . 24

3 Problem Statement and Methodology $\quad 27$

3.1 Problem Statement . . . . . . . . . . . . . . . . . . . 27

3.2 Research Methodology . . . . . . . . . . . . . . . . . . . 28

4 Experimental Approach $\quad 31$

4.1 Development Platform and Tools . . . . . . . . . . . . . . 32

4.2 Traffic Classification Datasets . . . . . . . . . . . . . . . 32

4.2.1 ISCX VPN-NonVPN Traffic Dataset . . . . . . . . . . . . 33

4.2 .2 NIMS Multi-Class Dataset . . . . . . . . . . . . . . 33

4.3 Data Preprocessing . . . . . . . . . . . . . . . . . 35

4.3 .1 Feature Selection . . . . . . . . . . . . . . . . 35

4.4 Architecture Characteristics \& Learning Setup . . . . . . . . . . 37

4.4 .1 C4.5 Architecture . . . . . . . . . . . . . . . 37

4.4 .2 KNN Architecture . . . . . . . . . . . . . . . 38

4.4 .3 ANN Architecture . . . . . . . . . . . . . . . 39 
4.4 .4 CNN Architecture . . . . . . . . . . . . . 40

4.4.5 RNN Architecture . . . . . . . . . . . . . . . . 40

4.5 Evaluation Criteria . . . . . . . . . . . . . . . . 41

5 ML vs DL in Adversarial-Free Environment 44

5.1 Evaluation of Feature Selection Experiment . . . . . . . . . . . . 44

5.2 Evaluating the Performance of ML Algorithms . . . . . . . . . . . 45

5.2.1 Performance of $\mathrm{C} 4.5 \ldots \ldots \ldots$. . . . . . . . . . . 45

5.2.2 Performance of $\mathrm{KNN}$. . . . . . . . . . . . . . . . . . 47

5.3 Evaluating the Performance of DL Algorithms . . . . . . . . . . . 49

5.3.1 Performance of ANN . . . . . . . . . . . . . . . 49

5.3.2 Performance of $\mathrm{CNN}$. . . . . . . . . . . . . . . 51

5.3.3 Performance of RNN . . . . . . . . . . . . . . . . 54

5.4 Performance Comparison of ML and DL . . . . . . . . . . . . 55

$6 \quad \mathrm{ML}$ and DL Resilience against Adversarial Evasion Attacks $\quad 61$

6.1 Evaluating ML Algorithms Resilience against Adversarial Attacks . . 62

6.1.1 Performance of $\mathrm{C} 4.5 \ldots \ldots \ldots 62$

6.1 .2 Performance of $\mathrm{KNN} \ldots \ldots \ldots$. . . . . . . . . . 64

6.2 Evaluating DL Algorithms Resilience against Adversarial Attacks . . 66

6.2.1 Performance of ANN . . . . . . . . . . . . . 66

6.2.2 Performance of $\mathrm{CNN}$. . . . . . . . . . . . . . 72

6.2.3 Performance of RNN . . . . . . . . . . . . . . 75

6.3 Performance Comparison of ML and DL in Adversarial Environment 77

7 ML and DL Resilience against Adversarial Evasion Attacks: Exper$\begin{array}{ll}\text { iments with Top Feature Selection } & 81\end{array}$ 
7.1 Evaluating ML Algorithms against Adversarial Attacks: Top Feature

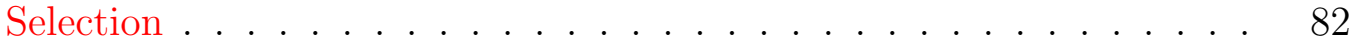

7.1 .1 Performance on $\mathrm{C} 4.5 \ldots \ldots \ldots \ldots$

7.1 .2 Performance on KNN . . . . . . . . . . . . . . . . 85

7.2 Evaluating DL Algorithms against Adversarial Attacks: Top Feature

Selection ........................... 87

7.2 .1 Performance on ANN . . . . . . . . . . . . . . 87

7.2 .2 Performance on CNN . . . . . . . . . . . . . . . 90

7.2 .3 Performance on RNN . . . . . . . . . . . . . . . . . . . 93

7.3 Performance Comparison of ML and DL in Adv. Environment using Top FS . . . . . . . . . . . . . . . . . . 96

8 Conclusions 101

8.1 Summary . . . . . . . . . . . . . . . . . . . . . 101

8.2 Future Work . . . . . . . . . . . . . . . . . . . . . . . . . 103

$\begin{array}{ll}\text { List of References } & 104\end{array}$

$\begin{array}{ll}\text { Appendix A } & 114\end{array}$ 


\section{List of Tables}

4.1 List of ISCX Encrypted Traffic . . . . . . . . . . . . . . . 34

4.2 List of NIMS Encrypted Traffic . . . . . . . . . . . . . . . 34

4.3 Training Parameters and Architecture in ANN Experiments . . . . 39

4.4 Training Parameters and Architecture in CNN Experiments . . . . 41

4.5 Training Parameters and Architecture in RNN Experiments . . . . 42

5.1 The 5 features selected from ISCX VPN-NonVPN and NIMS Datasets 45

5.2 Evaluation Metric Results (\%) of C4.5 models in adversarial-free environment for ISCX VPN-NonVPN . . . . . . . . . . . . 46

5.3 Evaluation Metric Results (\%) of C4.5 models in adversarial-free environment for NIMS dataset . . . . . . . . . . . . . . . . 47

5.4 Evaluation Metric Results (\%) of KNN models in adversarial-free environment for ISCX VPN-NonVPN . . . . . . . . . . . . . . 49

5.5 Evaluation Metric Results (\%) of KNN models in adversarial-free environment for NIMS dataset . . . . . . . . . . . . . 5 50

5.6 Evaluation Metric Results (\%) of ANN models in adversarial-free environment for ISCX VPN-NonVPN . . . . . . . . . . . . . 52

5.7 Evaluation Metric Results (\%) of ANN models in adversarial-free environment for NIMS dataset . . . . . . . . . . . . . . . 5 53 
5.8 Evaluation Metric Results (\%) of CNN models in adversarial-free environment for ISCX VPN-NonVPN . . . . . . . . . . . . 55

5.9 Evaluation Metric Results (\%) of CNN models in adversarial-free environment for NIMS dataset . . . . . . . . . . . . . 56

5.10 Evaluation Metric Results (\%) of RNN models in adversarial-free environment for ISCX VPN-NonVPN . . . . . . . . . . . . . 58

5.11 Evaluation Metric Results (\%) of RNN models in adversarial-free environment for NIMS dataset . . . . . . . . . . . . . .

6.1 Evaluation Metric Results (\%) of C4.5 models in adversarial attack environment for ISCX VPN-NonVPN . . . . . . . . . . . .

6.2 Evaluation Metric Results (\%) of C4.5 models in adversarial attack environment for NIMS dataset . . . . . . . . . . . . . .

6.3 Evaluation Metric Results (\%) of KNN models in adversarial attack environment for ISCX VPN-NonVPN . . . . . . . . . . . . . 67

6.4 Evaluation Metric Results (\%) of KNN models in adversarial attack environment for NIMS dataset . . . . . . . . . . . . . . . 68

6.5 Evaluation Metric Results (\%) of ANN models in adversarial attack environment for ISCX VPN-NonVPN . . . . . . . . . . . . 70

6.6 Evaluation Metric Results (\%) of ANN models in adversarial attack environment for NIMS dataset . . . . . . . . . . . . . . . . 71

6.7 Evaluation Metric Results (\%) of CNN models in adversarial attack environment for ISCX VPN-NonVPN . . . . . . . . . . . . 74

6.8 Evaluation Metric Results (\%) of CNN models in adversarial attack environment for NIMS dataset . . . . . . . . . . . . . . . . 75

6.9 Evaluation Metric Results (\%) of RNN models in adversarial attack environment for ISCX VPN-NonVPN . . . . . . . . . . . . . 
6.10 Evaluation Metric Results (\%) of RNN models in adversarial attack environment for NIMS dataset . . . . . . . . . . . . . 78

7.1 The Top 9 features selected from ISCX VPN-NonVPN and NIMS

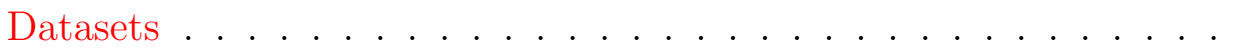

7.2 Evaluation Metric Results (\%) of C4.5 models in attack environment using Top FS for ISCX VPN-NonVPN . . . . . . . . . . . .

7.3 Evaluation Metric Results (\%) of C4.5 models in attack environment using Top FS for NIMS dataset . . . . . . . . . . . . . . . .

7.4 Evaluation Metric Results (\%) of KNN models in attack environment using Top FS for ISCX VPN-NonVPN . . . . . . . . . . . . .

7.5 Evaluation Metric Results (\%) of KNN models in attack environment using Top FS for NIMS dataset . . . . . . . . . . . . . . . .

7.6 Evaluation Metric Results (\%) of ANN models in attack environment using Top FS for ISCX VPN-NonVPN . . . . . . . . . . . . .

7.7 Evaluation Metric Results (\%) of ANN models in attack environment using Top FS for NIMS dataset . . . . . . . . . . . . .

7.8 Evaluation Metric Results (\%) of CNN models in attack environment using Top FS for ISCX VPN-NonVPN . . . . . . . . . . . . .

7.9 Evaluation Metric Results (\%) of CNN models in attack environment using Top FS for NIMS dataset . . . . . . . . . . . . . . . . .

7.10 Evaluation Metric Results (\%) of RNN models in attack environment using Top FS for ISCX VPN-NonVPN . . . . . . . . . . . .

7.11 Evaluation Metric Results (\%) of RNN models in attack environment using Top FS for NIMS dataset . . . . . . . . . . . . . . . . 97

A.1 ISCX VPN-NonVPN dataset Features based on the description in [1] 115

A.2 NIMS dataset Features based on the description in [2] [3] [4] . . . . . 116 


\section{List of Figures}

2.1 The Layered Architecture of ANN System . . . . . . . . . . . . 12

2.2 RNN Architecture. . . . . . . . . . . . . . . . . . . . 13

2.3 LSTM Unit Illustration . . . . . . . . . . . . . . . . . . . . . 14

4.1 Feature Selection Process . . . . . . . . . . . . . . . . . 36

5.1 C4.5 models trained by Adversarial-free ISCX VPN-NonVPN dataset 47

5.2 C4.5 models trained by Adversarial-free NIMS dataset . . . . . . . 48

5.3 KNN models trained by Adversarial-free ISCX VPN-NonVPN dataset 50

5.4 KNN models trained by Adversarial-free NIMS dataset . . . . . . . 51

5.5 ANN models trained by Adversarial-free ISCX VPN-NonVPN dataset 53

5.6 ANN models trained by Adversarial-free NIMS dataset . . . . . . . 54

5.7 CNN models trained by Adversarial-free ISCX VPN-NonVPN dataset 57

5.8 CNN models trained by Adversarial-free NIMS dataset . . . . . . 57

5.9 RNN models trained by Adversarial-free ISCX VPN-NonVPN dataset 59

5.10 RNN models trained by Adversarial-free NIMS dataset . . . . . . 60

5.11 Evaluation Metric Results (\%) of five models in adversarial-free environment. DL algorithms are shown in different shades of Blue. ML algorithms are shown in different shades of Red. . . . . . . . . . 60

6.1 Effect of Adversarial Samples generated on C4.5 models trained by Adversarial-free ISCX VPN-NonVPN dataset . . . . . . . . . . 63 
6.2 Effect of Adversarial Samples generated on C4.5 models trained by Adversarial-free NIMS dataset . . . . . . . . . . . . . 66

6.3 Effect of Adversarial Samples generated on KNN models trained by Adversarial-free ISCX VPN-NonVPN dataset . . . . . . . . . . . . 68

6.4 Effect of Adversarial Samples generated on KNN models trained by Adversarial-free NIMS dataset . . . . . . . . . . . . . . . .

6.5 Effect of Adversarial Samples generated on ANN models trained by Adversarial-free ISCX VPN-NonVPN dataset . . . . . . . . . . . . 71

6.6 Effect of Adversarial Samples generated on ANN models trained by Adversarial-free NIMS dataset . . . . . . . . . . . . . . . . 72

6.7 Effect of Adversarial Samples generated on CNN models trained by Adversarial-free ISCX VPN-NonVPN dataset . . . . . . . . . . 73

6.8 Effect of Adversarial Samples generated on CNN models trained by Adversarial-free NIMS dataset . . . . . . . . . . . . . . . 75

6.9 Effect of Adversarial Samples generated on RNN models trained by Adversarial-free ISCX VPN-NonVPN dataset . . . . . . . . . . . . . 79

6.10 Effect of Adversarial Samples generated on RNN models trained by Adversarial-free NIMS dataset . . . . . . . . . . . . . . . . 79

6.11 Effect of Adversarial Samples generated on five models trained by Adversarial-free ISCX VPN-NonVPN dataset. DL algorithms are shown in different shades of Blue. ML algorithms are shown in different shades of Red. . . . . . . . . . . . . . . . . .

6.12 Effect of Adversarial Samples generated on five models trained by Adversarial-free NIMS dataset. DL algorithms are shown in different shades of Blue. ML algorithms are shown in different shades of

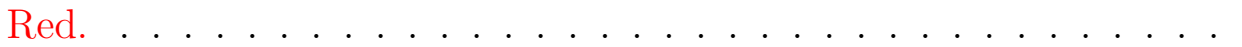


7.1 Effect of Adv. Samples generated on C4.5 models using Top FS trained by Adversarial-free ISCX VPN-NonVPN dataset . . . . . . . . . . . . 84

7.2 Effect of Adv. Samples generated on C4.5 models using Top FS trained by Adversarial-free NIMS dataset . . . . . . . . . . . . . . .

7.3 Effect of Adv. Samples generated on KNN models using Top FS trained by Adversarial-free ISCX VPN-NonVPN dataset . . . . . . . . . . . .

7.4 Effect of Adv. Samples generated on KNN models using Top FS trained by Adversarial-free NIMS dataset . . . . . . . . . . . . . . . .

7.5 Effect of Adv. Samples generated on ANN models using Top FS trained by Adversarial-free ISCX VPN-NonVPN dataset . . . . . . . . . . . .

7.6 Effect of Adv. Samples generated on ANN models using Top FS trained by Adversarial-free NIMS dataset . . . . . . . . . . . . . . . .

7.7 Effect of Adv. Samples generated on CNN models using Top FS trained by Adversarial-free ISCX VPN-NonVPN dataset . . . . . . . . . . . .

7.8 Effect of Adv. Samples generated on CNN models using Top FS trained by Adversarial-free NIMS dataset . . . . . . . . . . . . . .

7.9 Effect of Adv. Samples generated on RNN models using Top FS trained by Adversarial-free ISCX VPN-NonVPN dataset . . . . . . . . . . .

7.10 Effect of Adv. Samples generated on RNN models using Top FS trained by Adversarial-free NIMS dataset . . . . . . . . . . . . . . . .

7.11 Effect of Adversarial Samples generated on five models using Top FS trained by Adversarial-free ISCX VPN-NonVPN dataset. DL algorithms are shown in different shades of Blue. ML algorithms are shown in different shades of Red. . . . . . . . . . . . . . . . . . . . 
7.12 Effect of Adversarial Samples generated on five models using Top FS trained by Adversarial-free NIMS dataset. DL algorithms are shown in different shades of Blue. ML algorithms are shown in different shades of Red. . . . . . . . . . . . . . . . . . . . . . 100 


\title{
List of Abbreviations
}

\author{
ADAM Adaptive Learning Rate Algorithm \\ AI Artificial Intelligence \\ ANN Recurrent Neural Network \\ $C N N$ Convolutional Neural Network \\ $D L \quad$ Deep Learning \\ F1 F1-Score \\ FGSM Fast Gradient Sign Method \\ FNN Feed-Forward Neural Network \\ IDS Intrusion Detection System \\ $K N N \quad$ K-Nearest Neighbor \\ LSTM Long Short-Term Memory networks \\ $M L \quad$ Machine Learning \\ PGD Projected Gradient Descent \\ PR Precision Rate
}

xviii 
$R C \quad$ Recall Rate

ReLU Rectified Linear Unit(s)

$R N N \quad$ Recurrent Neural Network

SVM Support Vector Machine

TPR True Positive Rate

VPN Virtual Private Network

ZOO Zeroth Order Optimization 


\section{Chapter 1}

\section{Introduction}

This chapter covers an overview of the research in adversarial attacks in encrypted traffic classifications and the techniques used to carry out the research questions and contributions. Lastly, a summary of the thesis structure.

The characteristics of network topologies and Internet traffic vary depending on network environments and locations [5]. So, the Internet traffic supported in a simple home environment considerably differs from the enterprise network and we can consider the same for traffic applications being used and their critically too.

Network encrypted traffic classification is a key tool to monitor network activity and detect early threats that could threaten both regular and secured communications in any network. Network encrypted traffic can not be regarded as static as it changes over time and between networks [6]. Service providers, and more generally network administrators, have expressed their deep interest in being able to identify encrypted traffic for several reasons, such as, monitoring application trends, and ap-

plying policies depending on the traffic class. So, it became a challenge trying to classify encrypted traffic due to the evolution and fast emergence of applications. Artificial Intelligence (AI) techniques, such as Machine Learning and Deep Learning, can be applied to detect and characterize the encrypted traffic in the network. In 
this direction, ML and DL learning algorithms are used, where they can learn from the information contained in training data samples and process big data. Due to the rapid increase of using these algorithms and the volume of data generated and used through systems, the attack is becoming more sophisticated. According to the literature [7] [8] [9], there are various growing types of attacks targeting vulnerable ML and DL architecture designs.

The fact that the training of these algorithms is based on data, classification tasks can be carefully manipulated by crafted perturbations called adversarial samples, which can mislead any model towards inaccurate classification [10] [11]. Adversarial attacks are the method of adding noise to the original sample in such a way as to make the decision boundary between the regular and the crafted data shift and inaccurate. There are many types of adversarial attacks and algorithms to generate adversarial samples [10] [12] [13] [14] [15] [16] [17].

In this research, our primary goal is to study the effectiveness of the adversarial attacks against various state-of-the-art ML and DL models and their accuracy in classifying encrypted traffic. We achieve our goal by using multiple ML (C4.5 and $\mathrm{KNN})$ and DL (ANN, CNN, and RNN) models and investigate their resilience against

different state-of-the-art adversarial attacks (PGD, ZOO, and DeepFool) that have been recognized in different classification domains, such as in image classification and intrusion detection systems.

\section{$1.1 \quad$ Research Questions}

The thorough study of related work in adversarial attack and defence helped to derive several research questions:

1. How to build and train resistant ML and DL models in the adversarial-free 
environment to evaluate their resilience against adversarial attacks in classifying encrypted traffic?

2. Does use the top feature selection in each dataset improve our model's resilience against adversarial samples or not?

3. Which is more resilient in correctly classifying encrypted traffic against adversarial attacks, machine learning or deep learning?

\subsection{Thesis Contributions}

After reviewing the literature in the field of adversarial attack against encrypted traffic classifications, none of the current studies elaborated a comprehensive comparison between machine learning and deep learning in classifying encrypted multilabel traffic applications at adversarial-free and adversarial attack environments.

This research aims to study the resilience of using different learning techniques: Machine Learning (C4.5 and KNN) and Deep Learning (ANN, CNN, and RNN) in the encrypted traffic classification field through the aspect of resilient attack in an adversarial environment.

Several existing adversarial attack methods were proposed in the literature. We utilize three common state-of-the-art evasion attack methods, including Zeroth Order Optimization (ZOO), Projected Gradient Descent (PGD), and the DeepFool to generate adversarial samples. The results are discussed in Chapter 6 and 7.

To the best of our knowledge, we are the first to demonstrate a comprehensive comparison between machine learning and deep learning in classifying encrypted multilabel traffic applications in an adversarial-free environment and under the risk of adversaries from multiple state-of-the-art attack methods.

Our major contributions are elaborated below: 
- Firstly, to the best of our knowledge, this is the first research elaborates comprehensive comparison between machine learning $(\mathrm{C} 4.5, \mathrm{KNN})$ and deep learning (ANN, CNN, RNN) accuracy in classifying encrypted multilabel traffic applications on two benchmark datasets (ISCX VPN-NonVPN, NIMS) in an adversarial-free environment. The results show that on average, deep learning models perform better than machine learning models in terms of encrypted classification. Details in Chapter 5.

- Secondly, we investigate and evaluate each model's resilience against three stateof-the-art adversarial attacks (PGD, ZOO, and DeepFool) using the same top features. The result provides evidence that deep learning shows better resilience against adversarial samples classifying encrypted traffic in comparison to machine learning. Whereas, the impact of the attack varies depending on the type of attack. Evaluation of the model's resilience against multiple adversarial attacks is discussed in Chapter 6.

- Finally, we repeated the last two experiments using the top nine features generated from the feature selection technique. The results show that the model's resilience is substantially improved and attaining higher resilience compared to the results when using the same top features from Chapter 6. Details in Chapter 7.

\title{
1.3 Research Papers
}

This research produced the following research paper:

\author{
Evaluating Resilience of Encrypted Traffic Classification against Adver- \\ sarial Evasion Attacks
}


This paper [18] is published in IEEE ISCC 2021. This paper is a result of collaborations with Dr. Danish Sattar and Dr. Ashraf Matrawy. This paper is part of this thesis: Chapter 5 and 6 . The main purpose of this study is to investigate the effectiveness of different evasion attacks on two different benchmark datasets: ISCX VPN-NonVPN and NIMS and see how resilient machine and deep learning algorithms are, including C4.5, KNN, ANN, CNN, and RNN.

\subsection{Thesis Organization}

We structure the remainder of the thesis as follows. Chapter 2 begins with a background about Network Security, Machine and Deep learning models, and finally, an overview of the classification of adversarial samples, adversarial crafting methods, and adversarial threat models. That is followed by a literature review on machine learning, deep learning, in particular in encrypted traffic classification, and a summary of some related work on adversarial attacks. Chapter 3 states our research problem and our used methodology to carry out the research. In chapter 4, we describe the selected datasets and how we preprocess them using the feature selection techniques, followed by the architecture characteristics, learning setup, and evaluation criteria. Chapter 5 describes the performance of adversarial-free Machine learning and Deep learning models using the same top feature selection and performance comparison between the two learning models. Chapter 6 presents the experimental results and impact of using multiple adversarial attacks, followed by a performance comparison between machine and deep learning. In Chapter 7, we repeated the experiments done in the last two chapters (5 and 6) but using the top nine features selected for each dataset, and a quick comparison between the output and chapter's 5 and 6 results. Finally, in chapter 8 , we summarize our work, contributions, and the conclusion of our experiment 
results. Also, we present some recommendations on how we can strengthen our work in future research. 


\section{Chapter 2}

\section{Background}

\subsection{Network Security}

The Internet of Things, or IoT, is billions of physical devices that are now embedded in every part of our lives, connected to the internet, collecting and sharing data. IoT devices add so much to the home comforts. But all those appliances and devices that connect to the internet and each other on the home network have created new opportunities and offer new points of entry for cyberattacks [19]. A cyberattack is an attack launched by cybercriminals using different ways and techniques against single or multiple networks or computers. There are two types of cyberattacks, passive and active [20]. Passive cyberattack's purpose is to gain access to the computer system or network and to collect data without detection. Active cyberattack is often an aggressive attack where the attacker attempts to modify the data they have compromised to gain greater access to more restricted and valuable data. That makes an active attack more likely to be discovered compared to passive attacks.

Network security has become essential against cybercriminals in designing data protection and network integrity and usability. It takes preventative measures to protect the underlying networking infrastructure from unauthorized access, misuse, 
malfunction, modification, destruction, or improper disclosure [21]. Implementing these measures allows IoT devices, users and programs to perform their permitted critical functions within a secure environment.

\subsection{Machine Learning Models}

Machine Learning can be defined as an application of AI that gives computers the power to automatically extract information and enhance their performance from sample data or experience without any exploit programming [22]. It is a set of algorithms that can define data, learn from it, and then apply this gained knowledge to form smart decisions.

There is various type of learning paradigms derived from the type and volume of data. One of them is supervised learning, which begins with an established set of data and a certain understanding of how that data is classified. It is responsible for searching for patterns in data with labeled features that can be applied to an analytic process [22].

There are different types of machine learning networks, such as the C4.5 Decision Tree and K-Nearest Neighbor (KNN) algorithms, and all of them are supervised learning models. The following section focuses on machine learning architectures and algorithms.

\subsubsection{Decision Tree (C4.5)}

Decision trees are one of the most widely used classification techniques. A decision tree is a classifier expressed as a recursive partition of the training instances. It is constructed in a top-down manner, in each iteration, the instance space is partitioned by choosing the best attribute to split them [23]. 
The C4.5 algorithm is a machine learning algorithm that constructs a single decision tree, in which each internal node represents a test on features, each branch representing an outcome of the test, and each leaf node representing a class label [24]. Its accuracy level is high enough, independently of the data volume to be processed. Various studies identify the $\mathrm{C} 4.5$ algorithm as one of the top classifiers in data mining [25]. The C4.5 algorithm has the ability to handle an incomplete training dataset and to prune the resulting decision tree in order to reduce its size and optimize the decision path. C4.5 also has the ability to deal with continuous attributes.

\subsubsection{K-Nearest Neighbor (KNN)}

The KNN classifier is one of the simplest and basic machine learning algorithms based on the distance between data points in the vector space. It uses lazy learning for classification and is commonly referred to as a lazy classifier [26]. It can be used for both classifications as well as regression predictive problems. KNN works on a principle assuming every data point falling in near to each other is falling in the same class. In other words, it classifies a new data point based on similarity.

The advantage of using $\mathrm{KNN}$ is that it is robust to the noisy training data, as the amount of training data increases, the KNN becomes more effective and easy to implement in a multi-class problem. Its drawback is that the computation cost is high because of calculating the distance between the data points for all the training samples. Also, the KNN algorithm is very sensitive to outliers as it simply chose the neighbors based on distance criteria [27]. 


\subsection{Deep Learning Models}

On the other hand, one of the machine learning's members that carry out massive amounts of data and has significant learning flexibility by dividing its work as a nested hierarchy of concepts and each concept is defined with simpler concepts and so on, is the deep learning [28]. Unlike machine learning, it defeats the need for domain expertise as it works on learning high-level features from data in an incremental manner. Deep learning strength depends on some criteria starts with; How complex is the problem? What type of prediction is needed? How important is the accuracy to work vs. the interpretability? Will there be enough quality labelled data? How many resources will allocate for work?

Most deep learning models are built on neural networks and divided into three linear models of different layers [29]. The first layer is the input layer that provides the information from the outside world and passes on this information to the next layer called the hidden layer. This layer performs the needed computations and transfers the generated information to the output layer, which is responsible for showing the predicted results. The neural network structure is the same as a human brain, which is made up of cells called neurons, responsible for transmitting electrical signals to each other based on the received signals from other neurons [30].

There are different types of deep learning networks, such as Feed-forward (or Artificial) Neural Networks (ANN), Convolutional Neural Networks (CNNs), and Recurrent Neural Networks (RNNs), and all of them are supervised learning models. The following section focuses on deep learning architectures and algorithms. 


\subsubsection{Artificial Neural Network (ANN)}

ANN or known as a Feed-Forward Neural Network (FNN) processes input in a forwarding direction bypassing the input layer first, then the hidden layers, to the output layer, as shown in Figure 2.1. The ANN architecture consists of many neurons connected to other neurons, where each neuron connection is associated with weights to multiply with input variables (features). Weights are adjusted based on the input, the prediction output, and the activation function. The activation function has different selections, such as Sigmoid, Softmax, Tanh, and ReLU. In our work, we will use Softmax and ReLU as the activation functions in the hidden layers. The Softmax function is a combination of multiple Sigmoid functions that returns values in the range 0 to 1 [31]. While ReLU linear function is easy to compute and gives fast learning. It is widely used in the neural network because all the neurons are not activated at the same time, so when the output of linear transformation is zero, it deactivates a neuron [32].

The main advantage of using ANN is that it can map and learn any input to any output by using the activation function, where the system can approximate any function, regardless of its linearity. Due to additional hierarchy needs, it learns only one step at a time, therefore the learning time increases (almost) linearly in the number of levels in the model hierarchy, which is one of the ANN drawbacks [33].

\subsubsection{Convolutional Neural Networks (CNN)}

CNN is a subdomain for neural networks that are inspired by human brain neural networks. It is a technique inspired by the visual mechanisms of living organs and 


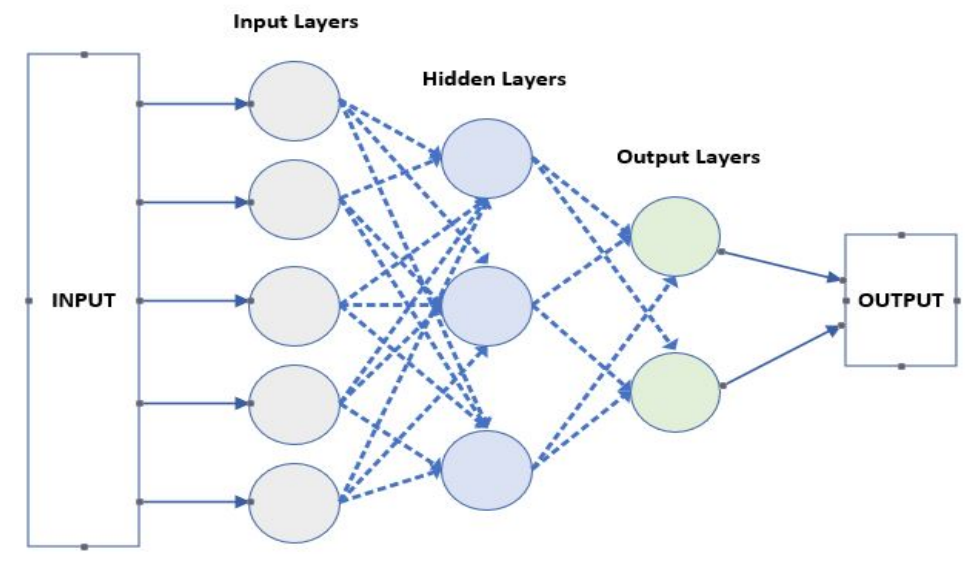

Figure 2.1: The Layered Architecture of ANN System

used with dimensional inputs. CNNs reduce the number of learning parameters significantly by using a set of convolution filters (kernels) in convolutional layers [34]. CNN is suitable for the following kinds of data: data in the form of multiple arrays; data that have strong local correlations; data whose features can appear anywhere; data in which objects are invariant to translations and distortions [35].

CNN architecture has various components, including convolution, pooling, and fully connected layers for sharing weight and pooling. The first layer is the convolution layer which emulates the behavior of the human eye, is connected to the input neurons in the first hidden layer. This layer applies a linear operation to extract input features and activation functions on 3D vectors. The pooling layer collects and concise the information received from the convolution layer. Since this layer reduces the dimension of the layer and parameter in training time, so it reduces the computational time accordingly. It is often added after a convolutional layer. The main pooling function used in the pooling layers is the Max Pooling. The final layer of the CNN is a Softmax layer, where the CNN layer flattens the output and passes it to the output layer for label prediction using the Softmax layer to emulate a probability distribution. 


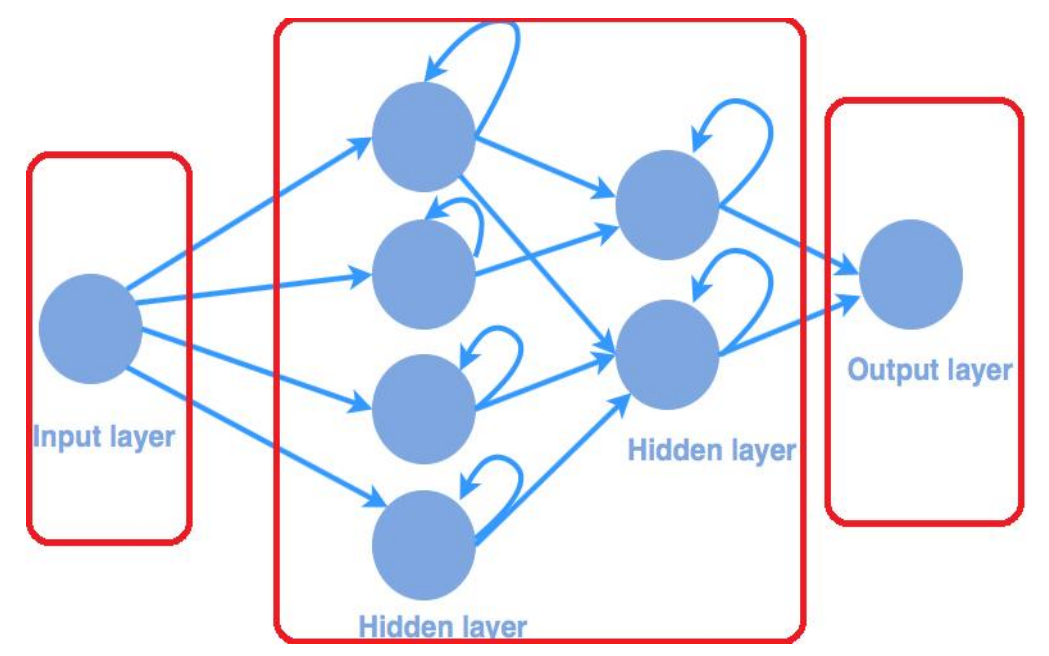

Figure 2.2: RNN Architecture

The main advantage of using CNN is learning the spatial pattern, which leads to learning complex patterns. CNN learns simple features at the first layers, and by the time it recognizes more complex features, and at the final layer, it recognizes objects. Another crucial point of this architecture is that neighboring neurons share portions of the receptive field combining percepts allows the extraction of general patterns.

\subsubsection{Recurrent Neural Networks (RNN) and LSTM}

RNN is are a class of neural networks that are used to classify series of data. It has both feed-forward and feed-back connections to the previous layers. Feed-back connections add a memory of past inputs where each unit receives the current and previous state where it feed-back the out to itself. RNN has a different learning phase from other neural network architectures, as it was designed to capture such correlation via a hidden layer, which keeps track of the context determined by the previous samples. RNN works similar to human brains where it does not start from scratch every time as it has a loop in its network that allows persisting information and share weight over time, as shown in Figure 2.2. 


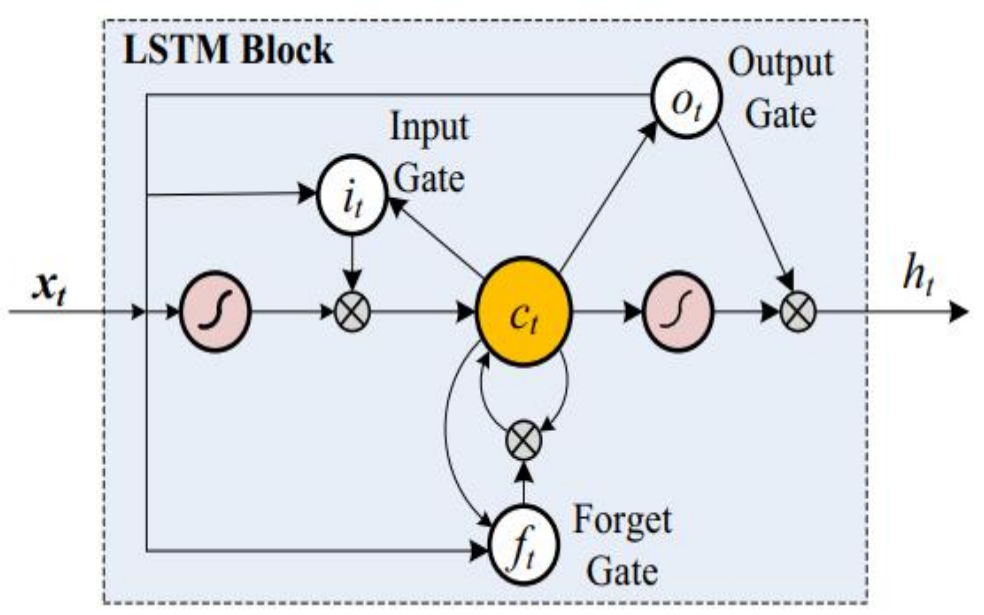

Figure 2.3: LSTM Unit Illustration

One of RNN's drawbacks is that its performance is poor when dealing with longterm dependencies as it does not learn how to successfully link with these dependencies. Another drawback is that it suffers from the vanishing gradient problem, which leads to insufficient and inaccurate results. To solve this problem, Long Short-Term Memory cells were introduced as RNN variation [36]. It can learn long-term dependencies and maintains the information for long-term periods. Several applications used it, such as speech recognition in smartphones, translation, image classification. LSTM has different layers as shown in Figure 2.3. Input Gate layer is the first layer where $i_{t}$ decides what information we are going to update. The second layer is the Forget Gate layer where $f_{t}$ decides what information to keep and which information to forget. The Output Gate is the final layer where $O t$ decides how much the internal state to transfer to the unit output. Outputs and cell memories $(c t)$ from last time step are connected to the three gates. With those layers, LSTM remembers all information through time. 


\subsection{Adversarial Attack Classification and Threat Model}

In this section, we focus on adversarial samples in the machine and deep learning algorithms and how they are designed to deceive these models to make a mistake. We will review the adversarial threat model and classification and explore the methodology of the state-of-art attack methods used to generate adversarial samples. At last, we will review some related work in adversarial attacks and encrypted traffic classification using machine learning and deep learning.

An adversarial attack is a purposeful attempt to deceive various machine learning algorithms [37] [38] [39]. At the same time, deep learning algorithms also have been proved to be vulnerable to adversarial samples causing deep learning to make predictions different from the ground truth with high confidence [15] [16] [40]. It employs the use of malicious samples that are crafted by an attacker to force a model to misclassify input data and fool the model by adding noisy perturbations to the original sample in such a way to make the decision boundary between the regular and the crafted data shift and inaccurate. It has become a real threat to modern technologies that rely on trained learning models to automate certain tasks. Identifying adversarial attacks remains a challenging problem due to the craftiness of adversarial samples generated by the attackers.

\subsubsection{Adversarial Samples}

We study adversarial samples used for classification, with final layers as Softmax activation functions. Adversarial samples were originally introduced by [7] as data points fed to a machine learning algorithm that is visually indistinguishable from 
"normal" examples but are specifically tuned to fool or mislead the machine learning system. These earliest forms of adversarial examples were framed as an intriguing property of neural networks where even a single, small gradient step was sufficient to harm the performance of deep learning classifiers [10] [11]. Adversarial samples can often be generated not only with access to the model's architecture and parameters but also without it, where such attacks would be called white-box and black-box attacks, respectively [41]. Therefore, it is important to understand the weaknesses of our models and come up with ways to defend them against potential adversaries.

\subsubsection{Adversarial Threat Model}

In this section, we focus on the adversarial machine threat model in terms of capabilities, knowledge, goal, timing, and limitations of an adversary [42] [43] [44].

\section{Adversarial Attack Surface}

An attacker can tamper with either the data inputs or the processing of data to confuse the target model, where the attack can be identified by the attack surface/time. We can classify adversarial attacks depending on the surface into two attacks: Evasion and Poisoning attack [41].

- Evasion Attack: Evasion is a most common attack on machine learning model performed during production. It exploits misclassifications but does not affect training. This type occurs at the decision time and alters the test set during the testing or prediction time [45] [46].

- Poisoning Attack: Poisoning is another category of attacks and can be considered as one of the most widespread. Poisoning can be different from evasion, where the adversary can poison the training dataset. To achieve this, the 
adversary derives and injects a point to decrease the classification accuracy. Poisoning attacks alter the training process through influence over the training data [46] [47].

\section{Adversarial Attack Capabilities}

Adversarial attack capabilities are determined by the amount of information available to an adversary about the system, which indicates the attack vector they may use on the threat surface. Adversarial capabilities in machine learning can be determined in different phases: training and testing phase [43].

While training the data, the attacker attempt to influence or corrupt the model directly by altering the dataset used for training by accessing partial or full training data considered the weakest attack on the training phase. Altering the model or input based on the adversarial capabilities depends on three broad attack strategies [43]:

- Data Injection: The adversary does not have any access to the training data or the learning algorithm but can insert adversarial samples to the training set and corrupt the target model.

- Data Modification: The adversary has full access to the training data only and not to the learning algorithm, where he can modify the data before used for training the target model.

- Logic Corruption: The adversary has to interfere with the learning algorithm, so it becomes very difficult to design a counter-strategy against these adversaries who can alter the learning logic. 


\section{Adversarial Attack Goal}

- Targeted Attack: The attacker aims to misclassify the predicted class of a benign input to a targeted class by crafting the benign input via adversarial perturbation [48].

- Misclassification Attack: The attacker aims to misclassify the benign input by adding adversarial perturbation so that the input predicted class is changed without a specific target class [49].

\section{Adversarial Attack Knowledge}

Adversarial attack efficiency and limitation are specified by the degree of information available to the attacker about the model at the attack time. It can be classified into White-box or Black-box attacks [50].

- White-box Attack: The adversary has total knowledge about the model, the algorithm used in training and can access the training data distribution.

- Black-box Attack: In this type of attack, the attacker does not know the model and uses information about the settings or past inputs to analyze the model vulnerability.

\subsubsection{Adversarial Samples Generation}

In this section, we present a state-of-the-art attack method used to generate adversarial samples in our research experiments, so that a classification model yields an adversarial output. The adversarial sample modification can be performed both in the training and testing phase. The robustness of the adversarial sample depends on the attacker's ability to find a sample relative to the original input. 


\section{Zeroth Order Optimization (ZOO)}

In [17], authors proposed this method to directly estimate the gradients of the targeted models for generating adversarial samples. The main goal of this method is to provide improved attacks to the targeted models, avoid the loss in attack transferability, and spare the need for training substitute models. Instead of training a substitute model, it directly approximates the gradient of the target model just based on the input data and output scores using stochastic gradient coordinate. Using this method proves the same effectiveness as the state-of-the-art white-box different attacks and exceeds other black-box attack methods. One of the advantages of this method that it can be applied to non-DL classifiers admitting the same input and output relationship as DL [17]. The ZOO attack decreases the probability of the original class and increases the probability of the target class, successfully attacking the model.

Given that $x$ denotes the adversarial example with a targeted class label $t$ toward misclassification, and $f(x, t)$ is the loss function that reflects the level of unsuccessful adversarial attacks. The loss function based on the output $F$ of a model is defined as:

$$
f(x, t)=\max \{\max \log [F(x)] i-\log [F(x)] t,-k\}
$$

Where $k$ is a tuning parameter for attack transferability and is set to zero for attacking a targeted model, and suggested large $k$ when performing transfer attacks. A well-trained model yields a skewed probability distribution on its output $F(x)$ such that the confidence score of one class significantly dominates the confidence scores of the other classes. The use of the log operator lessens the dominance effect while preserving the order of confidence scores due to monotonicity [17]. 


\section{Projected Gradient Descent (PGD)}

This type of adversarial attack attempts to find the perturbation that maximizes the loss of a model on a particular input while keeping the size of the perturbation smaller than a specified amount. Since it is a multi-step with a negative loss function, it overcomes the network overfit problem and became more robust than the Fast Gradient Sign Method (FGSM) adversarial samples, which comes from it [16]. In $\ell_{\infty}$-ball, PGD iterate to explore the maximum loss. The Resistance against PGD samples grants resistance against any first-order adversaries.

\section{DeepFool}

Moosavi et al. proposed [13] an algorithm that can compute adversarial examples and defeat state-of-art classifiers to change output prediction labels. It searches for the minimal perturbation to fool a classifier and uses concepts from geometry to direct the search. DeepFool attack is considered an efficient method to evaluate the robustness of classifiers. DeepFool was used in both models, binary and multi-class classifiers.

\subsection{Literature Review of Traffic Classification us- ing $\mathrm{ML}$}

Significant works have been surveyed in the field of machine learning-based IP traffic classification using anomaly detection techniques that do not rely on well-known port numbers, but statistical traffic characteristics [51].

One of the surveyed papers [52] presented a technique for clustering network flows without using packet payloads, but by taking few representative features from each 
flow, such as the total number of bytes, the number of exchanges between the endpoints, and the connection duration. They investigated a way to identify traffic with similar observable properties, applying an untrained machine learning classifier to this problem, where this classifier has the advantage of identifying groups/classes of Internet traffic using only transport layer characteristics. That was done without any direct assist in understanding what or why applications have been grouped this way. And, they did not evaluate the accuracy of the classification or explore which flow attributes produce the best results.

Erman et al. [53] addressed the challenge of traffic classification at the core of the network, where the network flows information and their contributors were limited at this period. They proposed a machine learning algorithm that uses the packets of a unidirectional TCP flow to estimate the flow statistics of the unobserved opposite direction. K-means algorithm was used for clustering, and they studied web, P2P and FTP traffic. The mapping method they used can merge the traffic clusters of known applications based on the payload of flows, but they cannot merge the traffic clusters of unknown applications. Their results demonstrated that with a sufficient set of labelled flows, the mapping method can achieve high accuracy.

Haffner et al. [54] proposed an approach for automated construction of application signatures (ACAS) by applying the supervised machine learning algorithms to automatically identify signatures for a range of applications. This technique uses features, such as flow duration, bytes per flow, packets per flow, and average packet size, for encrypted traffic, such as SSH and HTTPS, by extracting features from the unencrypted handshake that negotiate the encryption parameters of the TCP connection. The algorithms used to classify network traffic into different applications were AdaBoost, Hidden Markov, Naive Bayesian and Maximum Entropy models. Their results showed AdaBoost outperforms Naive Bayesian and Maximum Entropy with 
an $\mathrm{SSH}$ detection rate of $86 \%$ and a false positive rate of $0.0 \%$. The error rate is shown to be below 1\%. A major limitation of ACAS is that it assumes that the first 64 bytes of a flow completely describes the structure of the application protocol carried by the flow.

Moore et al. [55] proposed using a supervised machine learning technique to categorize Internet traffic by application. They used a Naive Bayes classifier with 248 full-flow-based features for training to classify network traffic based on flow statistical features. Selected traffic for Internet applications was grouped into different categories for classification, e.g. bulk data transfer, database, interactive, mail, services, WWW, P2P, attack, games and multimedia. The authors used Recall as evaluation metrics to evaluate the classifier's performance. The results showed that the Naive Bayes technique got high accuracy in network classifications where the Recall for the individual class of application ranged, for instance, from $98 \%$ for WWW to $90 \%$ for bulk data transfer, to approximately $44 \%$ for services traffic and $55 \%$ for P2P.

\subsection{Literature Review of Traffic Classification us- ing DL}

A comprehensive review of applications of deep learning methodologies has been done. And, the employed techniques for network traffic classification were investigated, besides discussing the advantages/disadvantages of the deep learning techniques used for network traffic monitoring applications [56].

Lotfollahi et al. [57] proposed a deep learning architecture that learns new features for each application from the packet content. The proposed architecture is called Deep Packet, which can handle both traffic characterization in the network traffic by categorizing it into major classes, such as email, chat, FTP, Skype, torrent, and P2P 
and application identification for recognizing end-user applications, such as Spotify, BitTorrent and Skype. They used a combination of a one-dimensional CNN and Stacked Auto-Encoders (SAE) for automatic feature extraction and classification of network traffic, for both application identification and traffic characterization, which is either encrypted or unencrypted traffic. Their framework reduces the amount of hand-crafted feature engineering and achieves great accuracy. The only drawback is that they extracted the first 1500 bytes only in the packets that may lead to uncertain results because there are some similar attributes in the same services.

Iliyasu et al. [58] introduced a deep learning technique for classifying on an enormous scale for encrypted communication of semi-supervised encrypted traffic to overcome the challenges associated with establishing ground truth labels of large encrypted traffic datasets. This technique was deep convolutional generative adversarial networks (DCGAN), where GAN is the technology that deploys the high machine speed for traffic encryption and imitates network traffic to solve problems and performs enormous amounts of calculations with encryption. Their work proposal was to use the samples generated by the DCGAN generator and the labeled data to improve the performance of the trained classifier on a small number of labeled samples, where their approach achieved good accuracy with these small labeled samples.

Wang et al. [35] proposed a one-dimensional convolutional neural network for the end-to-end encrypted traffic classification method. The method integrates feature extraction, feature selection and classifies the end-to-end framework by learning the nonlinear relationship between the original input and the expected output. They used a public ISCX VPN-nonVPN traffic dataset for verification. The proposed model has two convolutional, two pooling, and two fully connected layers. The bytes in each packet were normalized and the first 784 bytes were used by the model. In terms of classifying Non-VPN versus VPN, it achieved 100\% and 99\% Precision, and 99\% 
and $100 \%$ Recall, respectively. This method shows outstanding performance on both Non-VPN and VPN traffic, about 10\% higher than the C4.5 machine learning model that uses time series and statistical features. The authors' achievement using this method in VPN classification was due to the usage of training data and test data that are based on the same encryption method and encryption keys.

Alom et al. [59] proposed a deep learning model called Deep belief neural (DBN), which was based on Stack Restricted Boltzmann Machines (RBMs) for feature extraction and dimensionality reduction and based on SVM for the classification of network intrusion detection. The approach was implemented on merely $40 \%$ of the NSLKDD training dataset and did not use all the training datasets, where it explored the intrusion detection capabilities through a series of experiments. The trained DBN network can effectively identify unknown attacks provided to it, and the proposed system achieves $97.5 \%$ accuracy after 50 iterations. The authors report on the classification accuracy based on the test set comparing their approach with Support Vector Machines (SVM) and a hybrid version of DBN with SVM.

\subsection{Literature Review of Adversarial Attacks}

An adversarial attack is considered an attempt to deceive machine learning algorithms. It employs the use of malignant samples crafted by an attacker to force a model to misclassify a particular input or fool the model by adding noisy perturbations. Various studies were performed regarding adversarial attack samples and how they can mislead machine learning systems. Other researches focused on adversarial attack classes and their effectiveness on machine learning systems either as resilience or robustness. 


\section{Adversarial attacks and defences in images, graphs and text: A review}

$\mathrm{Xu}$ et al. [60] defined each type of adversarial attack and divided them into three categories: robust optimization techniques, gradient obfuscation methods, or adversarial example detection algorithms. Robust optimization techniques aim to improve the robustness of a classifier by learning model parameters by incorporating adversarial examples from a given attack into the training process. On the other hand, obfuscation methods rely on masking the input gradient needed by an attacker to construct adversarial examples. They focused on the adversarial examples of deep learning models and evaluated most research efforts on attacking various deep neural networks in different applications [60].

\section{Adversarial Examples: Attacks and Defences for Deep Learning}

Yuan et al. [12] focused on the adversarial examples on deep-learning models. They reviewed current research efforts on attacking various deep neural networks in different applications. They focused on explaining a single prediction in contrast to adversarial examples where the emphasis is on reversing the prediction. They also reviewed the robustness analysis of proposed schemes against noisy and maliciously generated inputs using adversarial attack algorithms. The defence methods are also extensively surveyed, where they proposed several detection and defence methods to mitigate the vulnerability induced by adversarial perturbation. 


\section{Adversarial attacks against intrusion detection systems: Taxonomy, solu- tions and open issues}

Corona et al. [61] presented an overview of adversarial attacks against intrusion detection systems (IDS). They provided a general taxonomy of attacks, IDS implementation weaknesses, and solutions for each mentioned attack. They highlighted the most promising research directions for the design of adversary-aware, harder to defeat IDS solutions. A high-level categorization of attack schemes through security event reconstruction for protecting against defence mechanism violations was discussed.

\section{Deep learning-based intrusion detection with adversaries}

Other researchers [9] proved that adversarial attacks are equally effective against deep learning models in network security applications on the NSL-KDD dataset. They also studied the impact of feature selection in generating adversarial examples and illustrated the vulnerability of deep learning algorithms against adversarial samples. They performed four white-box inference integrity feature vector attacks against trained classifiers on the NSL-KDD dataset: FGSM, JSMA, Deepfool, and CW, where the most effective attack was targeted FGSM and the least efficient was CW. 


\section{Chapter 3}

\section{Problem Statement and Methodology}

This chapter covers our research problem statement that carried out the purpose, scope, and thesis direction. We also present our methodology, including the encrypted traffic classification architecture and algorithm.

\subsection{Problem Statement}

A network encrypted traffic classification is a key tool to monitor the network activity and detect threats in a specific network [62]. Artificial Intelligence (AI) techniques, such as Machine Learning and Deep Learning, are increasingly used to detect and characterize the type of encrypted traffic in the network. The high volume of encrypted traffic increases the number and type of attacks and the challenges to label and classify the network traffic data. Adversarial samples have intentionally been designed to target a model to degrade performance and mislead any model towards inaccurate classification with high confidence [11] [10]. The linear nature of ML and DL is the main reason for its vulnerability against adversarial perturbation. The fact that adversarial samples can simply be generated using different algorithms can be used to perform attacks against various models that lead to misclassifying the input. 
Several types of research have been done in studying the effect of adversarial attacks in image classification [16], and Intrusion Detection Systems [9]. That served as a potential and motivation to explore machine learning and deep learning models, including $\mathrm{C} 4.5, \mathrm{KNN}, \mathrm{ANN}, \mathrm{CNN}$, and RNN, on different benchmark datasets and train them in an adversarial-free environment. After that, we can study the effectiveness of various well-known and state-of-the-art adversarial attacks to evaluate machine and deep learning algorithms' resilience.

Our primary goal is to explore and study several ML and DL architectures on two benchmark datasets: ISCX VPN-NonVPN and NIMS in the adversarial environment. To do that, we needed to concentrate on two main points: how to generate capable adversarial samples that can attack and deceive a model with small perturbation and how to train a model to be resilient against adversarial samples.

\subsection{Research Methodology}

We use the following methodology to carry out the research. We describe the details and justify our approach with our experimental results. In this research, we have conducted three main experiments, as this allows us to do a comprehensive analysis and comparison between these results. The experiments that we perform are presented in this research to analyze and compare different ML and DL model's effect in classifying encrypted traffic in an adversarial-free environment and their resilience against adversarial attacks on two benchmark datasets: ISCX VPN-NonVPN and NIMS. And also, compare the model's resilience against adversarial samples when using the same top features of both datasets and when using the top nine features for each dataset. In chapters 5,6 , and 7 , we present our experimental results to validate our selected approach. 
We start with a brief of our experiment methodology. We then describe the two datasets and how we preprocess them. The following section is about our model's architecture and learning setup for each set of experiments. Then we illustrate our experiment process.

1. We first split ISCX VPN-NonVPN and NIMS into training, testing, and validation sets.

2. We then preprocess the datasets: i) convert the non-numerical features, ii) normalize the datasets, apply feature selection using the Mutual Information technique, which we will use with the entire experiments in Chapters 5, 6 and 7.

3. We train the five models, two of ML (C4.5 and KNN) and three of DL (ANN, $\mathrm{CNN}$, and RNN), on the two adversarial-free datasets: ISCX VPN-NonVPN and NIMS using the same top features, as in Chapter 5.

4. We then used the three adversarial attack methods: PGD, ZOO, and DeepFool, to generate a collection of adversarial samples to analyze and compare the performance of machine learning and deep learning in an adversarial-free and adversarial attack environment for each dataset, respectively, as in Chapter 6.

5. In Chapter 7, we retrained the models by conducting the same sets of experiments using the top nine feature selections per each dataset, to evaluate the effectiveness of the generated adversarial samples from three evasion adversarial attack methods, and compare the model's resilient with the results from using the same top features in Chapter 6.

We denote the evasion attack methods as PGD, ZOO, and DeepFool. All experiments were performed on two encrypted traffic flow datasets and evaluated based on 
Precision, Recall, and F1-Score accuracy. All our evaluations performed using test data: 18,758 and 14,681 traffic flow on ISCX VPN-NonVPN and NIMS, respectively. In this work, we consider some assumptions about our attack threat model. The details of C4.5, KNN, ANN, CNN, and RNN, experiments are described in the sections below. The training process and evaluation work the same way across all our models. We will specify the training configuration in each experiment in terms of the selected optimizer, loss function, parameters, and metrics in Chapter 5 .

The attacks are evasion attacks, where an attacker has access to our model during prediction time that leads to misclassify the model decision, taking into considerations that a complete knowledge of the targeted models are known to perform a white-box attack with multi iterations. The adversarial threat model, including goal, capabilities, and knowledge of all the adversarial attacks are the same across all experiments. We use a sufficient number of samples in both datasets for training, validation and test. We split the training dataset into training and validation set to evaluate and assess the performance of the models during training time with $80 \%$ and $20 \%$. 


\section{Chapter 4}

\section{Experimental Approach}

We start with the platform we used to conduct the experiments. Then, we illustrate

the two datasets: ISCX VPN-NonVPN and NIMS, and how we preprocess them to be suitable for our experiments. After that, we will discuss our proposed model's architecture and how we set them up for the experiment operation.

In the next phase, we evaluated the effectiveness of using the adversarial evasion attack techniques to generate adversarial samples by performing several sets of experiments. That was done by retraining our models using the achieved feature selection data.

We use a sufficient number of samples in both datasets, and we split the dataset into training and validation set to evaluate the performance of the models with $80 \%$ and $20 \%$, respectively. We tried several data splitting such as $70 \%-30 \%, 75 \%-25 \%$, and also, $85 \%$ - 20\%. But, $80 \%$ - 20\% approach allows us to find the model's best hyper-parameter and also estimate the model's best performance and accuracy. We state the training design used in each experiment in terms of the selected optimizer, loss function, parameters, and metrics. 


\subsection{Development Platform and Tools}

Machine learning models were implemented using Scikit-learn, and deep learning models were executed using TensorFlow and Keras [63]. The experiments were performed on a 64-bit Windows machine with 16 GB memory and eight Intel core processors 3.00 GHz. We used the Scientific Python Development Environment (Spyder) [64], included with the Anaconda platform, to write and run our code from there. We used the open-source IBM Robustness Toolbox (ART) framework for generating the adversarial samples [65]. ART is a Python library that provides algorithms to build defences against several machine learning models and test them against adversarial threats.

Each evasion attack algorithm found in the ART framework has its own parameters, i.e., type of classifier used, the maximum number of iterations, the batch size on which the samples are generated, and the number of class gradients to compute, where each parameter in every attack are modified according to the attacked model and the number of classes in each dataset. Several experiments and trials have been done on each attack's parameter to get the best adversarial attack results, to accurately test the trained model's performance. After splitting the dataset, we provided the attack algorithm with the $20 \%$ test data to add noisy perturbations to these original samples and then push it to our trained models to check their accuracy and resilience against those adversarial samples in accurately classifying the encrypted traffic applications.

\subsection{Traffic Classification Datasets}

Since the performance of machine learning and deep learning algorithms depends on the dataset to some extent, classifier accuracy differs between various datasets. 
Therefore, it is significant to study the dataset carefully as part of the learning approach. Generally, the dataset used for encrypted traffic classifications is flow-based. In our research, we use two datasets: ISCX VPN-NonVPN and NIMS.

\subsubsection{ISCX VPN-NonVPN Traffic Dataset}

ISCX VPN-NonVPN traffic Dataset [1] is a captured network encrypted traffic set consisting of different types of traffic and applications. There are two data formats in this encrypted traffic dataset, labeled flow features (ARFF format) and unlabeled raw traffic (PCAP format). It contains packets captured over Virtual Private Network (VPN) sessions and regular encrypted sessions (Non-VPN). The dataset objective was to identify different types of applications using VPN and Non-VPN sessions. The flow-based classification method (such as flow bytes per second, duration per flow, etc.) was used to characterize Non-VPN and VPN encrypted traffic using only time-related features. Each row in the dataset has 24 features, including class labels, and has more than eighteen thousand records.

The ISCX dataset has fourteen application labels (or classes), where seven of them were collected using VPN sessions, and the same seven were collected using regular Non-VPN encrypted sessions, as shown in Table 4.1. We use in our experiments 15,005 records for training and 3,752 for testing. The ISCX VPN-NonVPN dataset is a combination of both VPN and Non-VPN encrypted sessions. Table A.1 in the Appendix provides a summary of dataset features.

\subsubsection{NIMS Multi-Class Dataset}

NIMS Dataset [2] [3] [4] is a captured network encrypted traffic set consisting of packets collected internally at the authors' research testbed network. The dataset is 
Table 4.1: List of ISCX Encrypted Traffic

\begin{tabular}{||l|l||}
\hline Traffic Type & \multicolumn{1}{|c||}{ Content } \\
\hline \hline Browsing & Firefox, Chrome \\
\hline Chat & ICQ, AIM, Skype, Facebook, Hangouts \\
\hline Streaming & Vimeo, Youtube, Netflix, Spotify \\
\hline Mail & Email, Gmail ( SMPT, POP3, IMAP ) \\
\hline VoIP & Facebook, Skype, Hangouts, VoipBuster \\
\hline P2P & uTorrent, Bittorrent \\
\hline File Transfer & Skype, FTPS, SFTP \\
\hline
\end{tabular}

Table 4.2: List of NIMS Encrypted Traffic

\begin{tabular}{||l||}
\multicolumn{1}{||c||}{ Traffic Type } \\
\hline \hline Local Tunneling \\
\hline Remote Tunneling \\
\hline SCP \\
\hline SFTP \\
\hline X11 \\
\hline Shell \\
\hline
\end{tabular}

available in two data formats, ARFF format and CSV format. The dataset is labeled. Different network scenarios are emulated using one or more computers to capture the resulting traffic. SSH connections are generated by connecting a client computer to four SSH servers outside the testbed via the Internet. Each row in the dataset has 23 features, including class labels, and has more than fourteen thousand records. It has six classes of encrypted SSH labels, as shown in Table 4.2. In our experiments, we use 11,408 records for training and 2,936 for testing. Table A.2 in the Appendix provides a summary of dataset features. 


\subsection{Data Preprocessing}

Data preprocessing is a necessary step before building a model and feeding it with the data. Our datasets have different scales and significant deviations that resulted in a weak performance model and slow training time. To prevent that, we normalized the dataset to change the values of the numeric features that have a different range to increase classifier performance and accuracy using StandardScaler [66]. It helped in scaling the data within a range to avoid building incorrect models while training and/or executing data analysis. After that, we reshaped the train and test input for CNN and RNN models into three dimensions (3D) using reshape() function [67]. In our experiment, we reshaped the input for the ISCX VPN-NonVPN dataset into a matrix of size $(15005 \times 5 \times 1)$ for the training set and size of $(3752 \times 5 \times 1)$ for the testing set. For the NIMS dataset, we reshaped the input into a matrix of size (11408 $\mathrm{x} 5 \times 1)$ for the training set and size $(2936 \times 5 \times 1)$ for the testing set.

\subsubsection{Feature Selection}

ISCX VPN-NonVPN and NIMS datasets have a large number of features, and many of them are insignificant and redundant, which may lead to prediction process inefficiency due to their low availability in prediction time compared to other essential features. Sometimes they lead to high training time consumption and overfitting. That is why it is mandatory to select essential features and enhance the model performance in respect of prediction accuracy and a faster prediction process. So, we used the feature selection methods to reduce the model complexity as shown in Figure 4.1.

There are different types of feature selection methods, such as wrapper, intrinsic, and filter methods [68]. Wrapper feature selection methods generate many models 


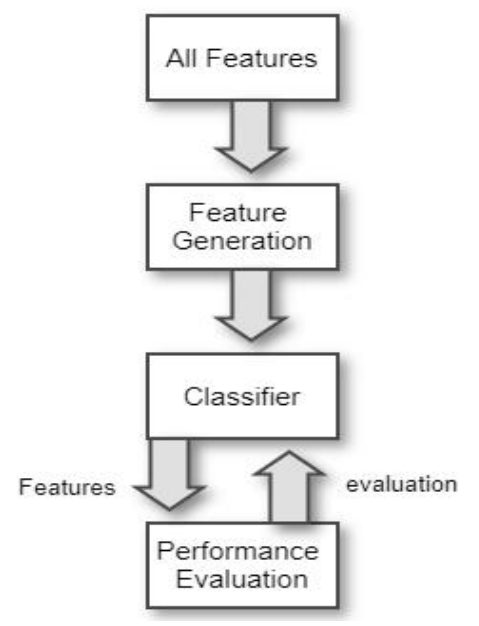

Figure 4.1: Feature Selection Process

with different subsets of input features and get the best-performing model by selecting those features. While intrinsic feature selection methods are machine learning algorithms that perform automatic feature selection during training as part of learning the model. Regularization and tree-based methods are good examples of an intrinsic feature selection method. Finally, filter feature selection methods use statistical measures to score the correlation and evaluate the relationship between the input variable and the target variable that can be filtered to choose those input variables that will be used in the model. A good example of a filter feature selection method is the correlation filter method. In our experiment, we focus on exploring the best feature selection methods that can achieve high accuracy and better performance for our models. We use a multilabel classification technique called mutual information [69]. Mutual information is a measure of statistical independence with two main properties: it can measure any relationship between random variables, including nonlinear relationships, and is invariant under transformations in the feature space invertible and differentiable [70]. In Chapter 5, we analyzed the experimental details and results of feature selection techniques. 


\subsection{Architecture Characteristics \& Learning Setup}

In the section, we explore the selected parameters for building two machine learning, i.e., C4.5 and KNN, and three deep learning models, i.e., ANN, CNN, and RNN, in consideration of selecting the best hyper-parameters that have a significant impact on model performance. We use different parameters in building our neural network architecture, such as the number of hidden layers, learning rates, flatten, and dropout. The number of hidden layers is selected usually depending on the dataset size and dimensions. So, increasing the number of neurons in hidden layers will guarantee strong neural networks but at the expense of longer model training time. Also, since the learning rate manages the number of weights alteration, so it should be small to reach acceptable results.

\subsubsection{C4.5 Architecture}

It generates a decision tree for classification. It can accept data with categorical or numerical values as it uses information gain as splitting criteria [71]. The algorithm uses a training set containing interpreted and classified instances with any number of features and a class attribute. The algorithm selects the feature from the remaining feature set at every step, that splits the instances in the best way based on the statistical measure information gain. After determining the best feature, all instances get split into two or more smaller subsets using the selected feature as the splitting criterion. This split is represented by a single node in the decision tree that branches out for every resulting split set. The instances that cannot be split again by the used feature, are removed from the corresponding feature set. That is the case with binary 
features, where this procedure is repeated over and over for every subset, until only a single instance is left in a subset or another stopping criterion is met, thus creating a leaf node containing the remaining instances. This creates a decision tree with nodes and leafs. The multi-classification of new instances with the resulting decision tree is very easy. Starting with the first root node, the instance is tested for the feature that was used in the corresponding decision node, and the appropriate branch is selected. There, the following node determines the next test. This is repeated until a final leaf node is reached, and each leaf node determines the classification result of the prompted instance depending on the class attribute of the instances that remained there in the training process. This class attribute is predicted for the new instance.

\subsubsection{KNN Architecture}

It is an algorithm based on a distance function that measures the difference or similarity between two instances [72] by storing all the available data. In other words, KNN works on a principle assuming every data point falling in near to each other is falling in the same class. In the case of classification, the algorithm starts to find the distances between a query and all the examples in the data, selecting the specified number of examples $(\mathrm{K})$ closest to the query, then votes for the most frequent label. To identify and select the closest neighbors to the input instance, the Euclidean method is the standard measure used in the KNN algorithm to determine the distance between instances. It uses lazy learning for classification and is sometimes referred to as a lazy classifier. It can perform both binary and multiclass classification. 
Table 4.3: Training Parameters and Architecture in ANN Experiments

\begin{tabular}{||l|l||}
\hline Parameter & Value \\
\hline \hline No. of hidden layers & 2 \\
\hline Layer 1 & 180 neurons \\
\hline Layer 2 & 160 neurons \\
\hline Dropout & 0.2 \\
\hline Optimizer & ADAM \\
\hline Activation function & ReLU and Softmax \\
\hline Learning rate & 0.01 \\
\hline Epoch & 20 \\
\hline Batch Size & 64 \\
\hline
\end{tabular}

\subsubsection{ANN Architecture}

In ANN experiments, we use the same architecture on both ISCX VPN-NonVPN and NIMS datasets. We slice the data into batches with a batch size of 64 and repeatedly over the entire dataset over twenty epochs to train the model. We use ADAM optimizer for its computational efficiency since it works well on problems with noisy and large datasets, and over that, it requires little memory space only. Our network parameters and architecture listed in Table 4.3.

We use these parameters to run our ANN experiments for classifying the encrypted traffic on the two datasets. First, we train the model using the original dataset to built an adversarial-free ANN model. Then, we attack the adversarial-free ANN models using the adversarial evasion attacks. 


\subsubsection{CNN Architecture}

We use the same CNN architecture and the same hyper-parameters on both ISCX VPN-NonVPN and NIMS datasets. We use two types of one-dimensional layers in our CNN architecture: Convolution and Max Pooling layers. Our CNN architecture has two Conv1D that extract features from the packet flow. It also has two MaxPooling1D that apply dimensionality reduction to reduce the size of the inputs and decrease computation time. We use three fully connected layers with the ReLU activation function and one output layer with a softmax activation layer to classify the input according to each dataset category. We use a fatten layer to fatten the input shapes and Dropout layers to prevent overfitting in the model during training time. We then use the ADAM optimizer in our CNN model, and we slice the data into batches with a size of 64 and repeatedly over the entire dataset over twenty epochs to train the model. Our network parameters and architecture listed in Table 4.4.

We use these parameters to run our CNN experiments for classifying the encrypted traffic on the two datasets: ISCX VPN-NonVPN and NIMS. First, we train the model using the original dataset to built an adversarial-free ANN model. Then, we attack the adversarial-free CNN models using the adversarial evasion attacks.

\subsubsection{RNN Architecture}

We use the same RNN architecture with LSTM for long-term learning and the same LSTM architecture on both ISCX VPN-NonVPN and NIMS datasets. Our RNN network architecture uses two LSTM layers with a sigmoid activation function and one fully connected layer with a ReLU activation function. And one output layer with a sigmoid activation layer to classify input according to each dataset category. 
Table 4.4: Training Parameters and Architecture in CNN Experiments

\begin{tabular}{||l|l||}
\hline Parameter & Value \\
\hline \hline Convolution layer & 2 \\
\hline Layer 1 & 84 neurons \\
\hline Layer 2 & 64 neurons \\
\hline Max Pooling layer & 2 \\
\hline Dropout & 0.2 \\
\hline Optimizer & ADAM \\
\hline Activation function & ReLU and Softmax \\
\hline Learning rate & 0.01 \\
\hline Epoch & 20 \\
\hline Batch Size & 64 \\
\hline
\end{tabular}

We use Dropout layers to prevent overfitting in the model during training time. We then use the ADAM optimizer in our RNN model, and we slice the data into batches with a size of 64 and repeatedly over the entire dataset over twenty epochs to train the model. Our network parameters and architecture listed in Table 4.5.

We use these parameters to run our RNN experiments for classifying the encrypted traffic on the two datasets: ISCX VPN-NonVPN and NIMS. First, we train the model using the original dataset to built an adversarial-free ANN model. Then, we attack the adversarial-free RNN models using the adversarial evasion attacks.

\subsection{Evaluation Criteria}

To help evaluate and compare the effectiveness of using different classifiers in the adversarial-free environment and examine their resilience against adversarial attacks, 
Table 4.5: Training Parameters and Architecture in RNN Experiments

\begin{tabular}{||l|l||}
\hline Parameter & Value \\
\hline \hline LSTM layer & 2 \\
\hline Layer 1 & 84 neurons \\
\hline Layer 2 & 64 neurons \\
\hline Dropout & 0.25 \\
\hline Optimizer & ADAM \\
\hline Activation function & ReLU and Sigmoid \\
\hline Learning rate & 0.01 \\
\hline Epoch & 20 \\
\hline Batch Size & 64 \\
\hline
\end{tabular}

we will present the evaluation metrics we use in all experimental phases. To evaluate the model performance, various metrics can be used, including model Precision Rate (PR), Recall Rate (RC), and F1-Score (F1), which are commonly used in the literature.

Now, we give an example of what we mean by TP, FP, and FN as commonly used in the literature. If we are talking about Browsing, $\mathrm{TP}$ is the number of instances correctly classified as Browsing. FP is the number of instances incorrectly classified as Browsing, and FN will be the case where Browsing be incorrectly classified as something else (e.g. Chat) [9].

The following metrics formulas are based on True/False-positive and Falsenegative [9].

- Precision: The ratio of real positive samples over the total predicted positive samples [9]. 


$$
\text { Precision }=\frac{T P}{T P+F P}
$$

- Recall: It is also called True Positive Rate (TPR) or detection rate. It is the total number of True Positives (TP) among all actual positive samples [9].

$$
\text { Recall }=\frac{T P}{T P+F N}
$$

- F1-Score: It is a measure of the model's accuracy on a dataset. It evaluates the binary classification systems, which classify examples as positive or negative. The F1-score is a way of combining the Precision and Recall of the model and is defined as the harmonic mean of the model's Precision and Recall [9].

$$
F 1 \text { Score }=2 * \frac{\text { Precision } * \text { Recall }}{\text { Precision }+ \text { Recall }}
$$

The F1-Score metric was used to weigh the two ratios (Precision and Recall) in a balanced way since we have uneven class distribution. If the cost of false positives and false negatives are very different, it is better to look at both Precision and Recall. 


\section{Chapter 5}

\section{ML vs DL in Adversarial-Free Environment}

In this chapter, we build a highly accurate model on ISCX VPN-NonVPN and NIMS for each architecture model, i.e., C4.5, KNN, ANN, CNN, and RNN. The experimental setup was repeated several times to obtain the finest accuracy using the feature selection technique: Mutual Information. We compare the accuracy of ML and DL models using the same top features from both datasets. We will discuss the accuracy results generated when the classifiers were applied on the two selected datasets: ISCX VPN-NonVPN and NIMS. We train each model on the same $80 \%$ of the dataset and conduct the validation on the remaining $20 \%$, respectively. The performance of each architecture is described in the following sections.

\subsection{Evaluation of Feature Selection Experiment}

We focus on using a multi-classification feature selection method that can be applied to achieve high Precision and Recall accuracy and better performance. We used the Mutual Information technique.

We train the C4.5, KNN, ANN, CNN, and RNN using Mutual Information for 
ISCX VPN-NonVPN and NIMS. The Experiment was executed six times for each algorithm with different batches and epoch values to obtain the best accuracy. After various tries and observations, we improve our model performance by selecting the same top five features for each dataset listed in Table 5.1.

Table 5.1: The 5 features selected from ISCX VPN-NonVPN and NIMS Datasets

\begin{tabular}{||c|l|c|l||}
\hline ID & ISCX & ID & NIMS \\
\hline \hline 0 & duration & 16 & duration \\
5 & max-fiat & 10 & max-fiat \\
6 & max-biat & 14 & max-biat \\
7 & mean-fiat & 9 & mean-fiat \\
8 & mean-biat & 13 & mean-biat \\
\hline
\end{tabular}

\subsection{Evaluating the Performance of ML Algorithms}

\subsubsection{Performance of C4.5}

We report C4.5 models' performance and discuss their capability to learn encrypted traffic flow characterization and application identification on ISCX VPN-NonVPN and NIMS datasets. Table 5.2 and Table 5.3 shows the performance metrics results for the ISCX VPN-NonVPN and NIMS categorical classification in an adversarialfree environment to assist in understanding and comparing the model performance. The result values are rounded to the nearest integer. Figure 5.1 and 5.2 show the F1-Score performance metrics results for each application that was calculated from the Precision and Recall results in Table 5.2 and Table 5.3.

In ISCX VPN-NonVPN, Figure 5.1 illustrates that VoIP has the highest and 
almost the same F1-Score accuracy of $98 \%$ in both VPN and Non-VPN over the other applications. Also, we can see that Chat is getting the lower F1-Score results of $42 \%$ in both VPN and Non-VPN classification.

Regarding the NIMS in Figure 5.2, we can see that RT and X11 prediction have the highest F1-Score results of $98.5 \%$ and $97.5 \%$, respectively, compared to LT and SFTP where their prediction was $85.5 \%$ and $91.5 \%$, respectively.

Table 5.2: Evaluation Metric Results (\%) of C4.5 models in adversarial-free environment for ISCX VPN-NonVPN

\begin{tabular}{||l|c|c||}
\hline Class & \multicolumn{2}{|c||}{ Clean Data } \\
\hline \hline- & Precision & Recall \\
\hline Browsing & 64 & 65 \\
\hline Chat & 43 & 40 \\
\hline Streaming & 53 & 53 \\
\hline Mail & 50 & 50 \\
\hline VoIP & 99 & 98 \\
\hline P2P & 68 & 71 \\
\hline File Transfer & 69 & 54 \\
\hline VPN-Browsing & 62 & 61 \\
\hline VPN-Chat & 42 & 42 \\
\hline VPN-Streaming & 76 & 82 \\
\hline VPN-Mail & 55 & 60 \\
\hline VPN-VoIP & 99 & 97 \\
\hline VPN-P2P & 48 & 61 \\
\hline VPN-File Transfer & 58 & 57 \\
\hline Average & 63 & 64 \\
\hline
\end{tabular}


Table 5.3: Evaluation Metric Results (\%) of C4.5 models in adversarial-free environment for NIMS dataset

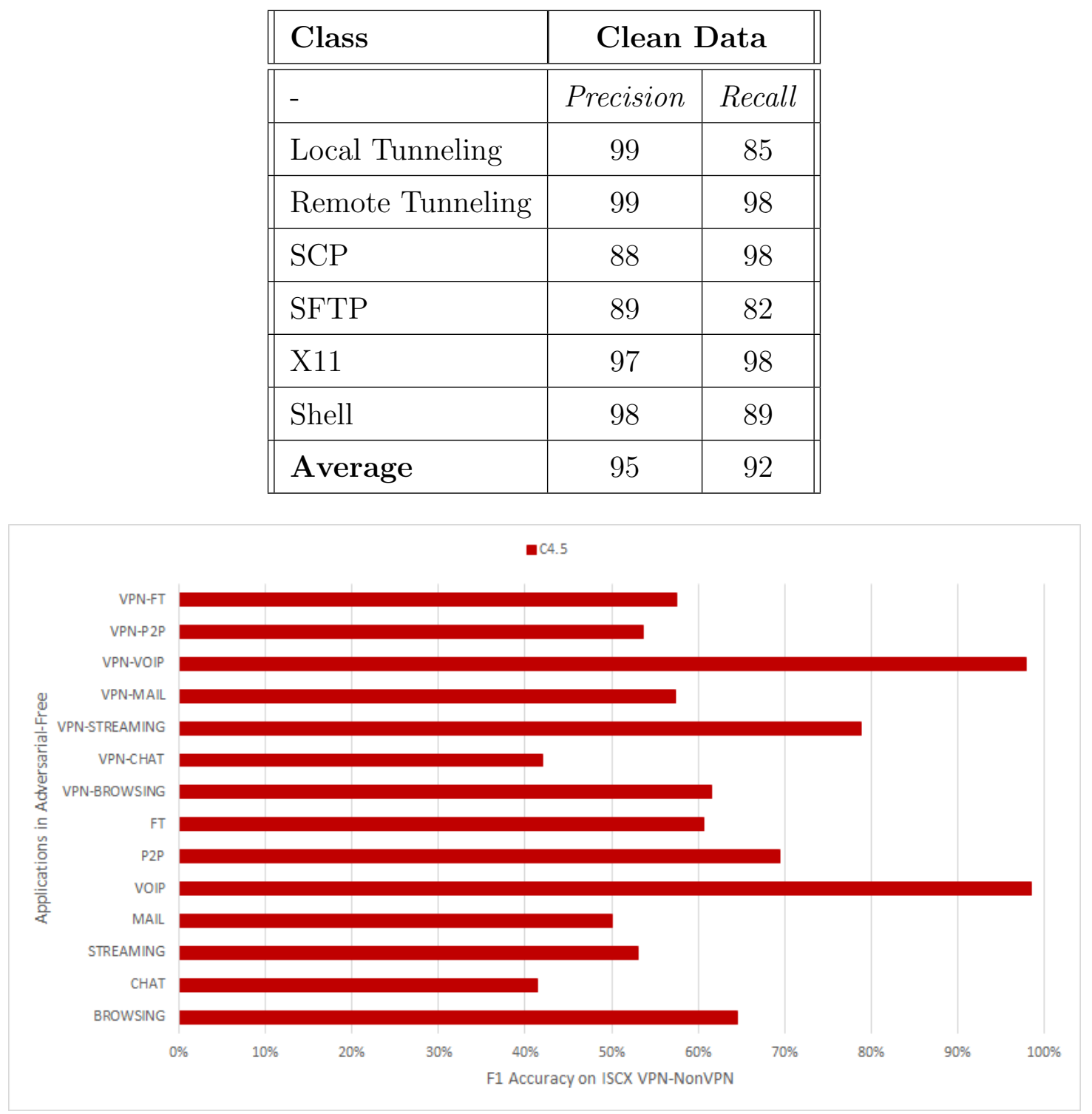

Figure 5.1: C4.5 models trained by Adversarial-free ISCX VPN-NonVPN dataset

\subsubsection{Performance of KNN}

In this section, we evaluate the performance and capabilities of the KNN models to learn encrypted traffic flow characterization and application identification on ISCX 


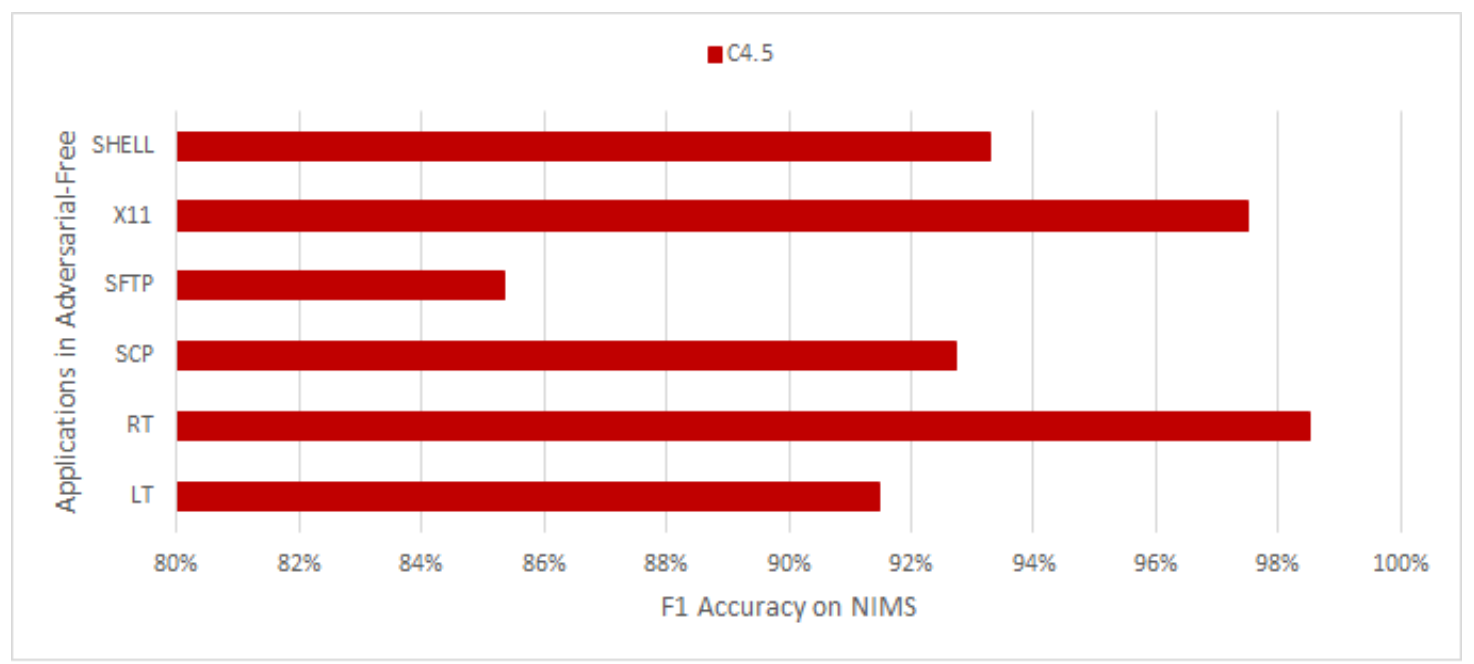

Figure 5.2: C4.5 models trained by Adversarial-free NIMS dataset

VPN-NonVPN and NIMS datasets. Table 5.4 and Table 5.5 presents the generated metrics results in classifying the applications of ISCX VPN-NonVPN and NIMS datasets to help in evaluating the KNN model's performance. The result values are rounded to the nearest integer. We can see the F1-Score accuracy results for each application in Figure 5.3 and 5.4.

Similar to C4.5 results in ISCX VPN-NonVPN, Figure 5.3 indicates that KNN models lead to high F1-Score accuracy prediction for VoIP application in both VPN and Non-VPN traffic of almost 95\%. But, Browsing and Streaming accuracy prediction was improved in VPN traffic from $68 \%$ and $80 \%$, respectively, compared to Non-VPN traffic, which was $64 \%$ and $52 \%$, respectively.

In NIMS, the F1-Score of LT and RT was accurately predicted by $98.5 \%$ and $99 \%$ compared to SCP where its accuracy was less by 15\%, as shown in Figure 5.4. 
Table 5.4: Evaluation Metric Results (\%) of KNN models in adversarial-free environment for ISCX VPN-NonVPN

\begin{tabular}{||l|c|c||}
\hline Class & \multicolumn{2}{|c|}{ Clean Data } \\
\hline \hline- & Precision & Recall \\
\hline Browsing & 64 & 65 \\
\hline Chat & 54 & 51 \\
\hline Streaming & 53 & 52 \\
\hline Mail & 55 & 46 \\
\hline VoIP & 96 & 97 \\
\hline P2P & 71 & 70 \\
\hline File Transfer & 67 & 52 \\
\hline VPN-Browsing & 69 & 67 \\
\hline VPN-Chat & 46 & 48 \\
\hline VPN-Streaming & 74 & 86 \\
\hline VPN-Mail & 52 & 60 \\
\hline VPN-VoIP & 96 & 94 \\
\hline VPN-P2P & 55 & 40 \\
\hline VPN-File Transfer & 49 & 60 \\
\hline Average & 64 & 63 \\
\hline
\end{tabular}

\subsection{Evaluating the Performance of DL Algorithms}

\subsubsection{Performance of ANN}

In this section, we study and report the ANN model's effectiveness in learning encrypted traffic flow characterization and application identification on ISCX VPNNonVPN and NIMS datasets. From Table 5.6 and Table 5.7, we can see the Precision and Recall results for the ANN accuracy performance in ISCX VPN-NonVPN and 
Table 5.5: Evaluation Metric Results (\%) of KNN models in adversarial-free environment for NIMS dataset

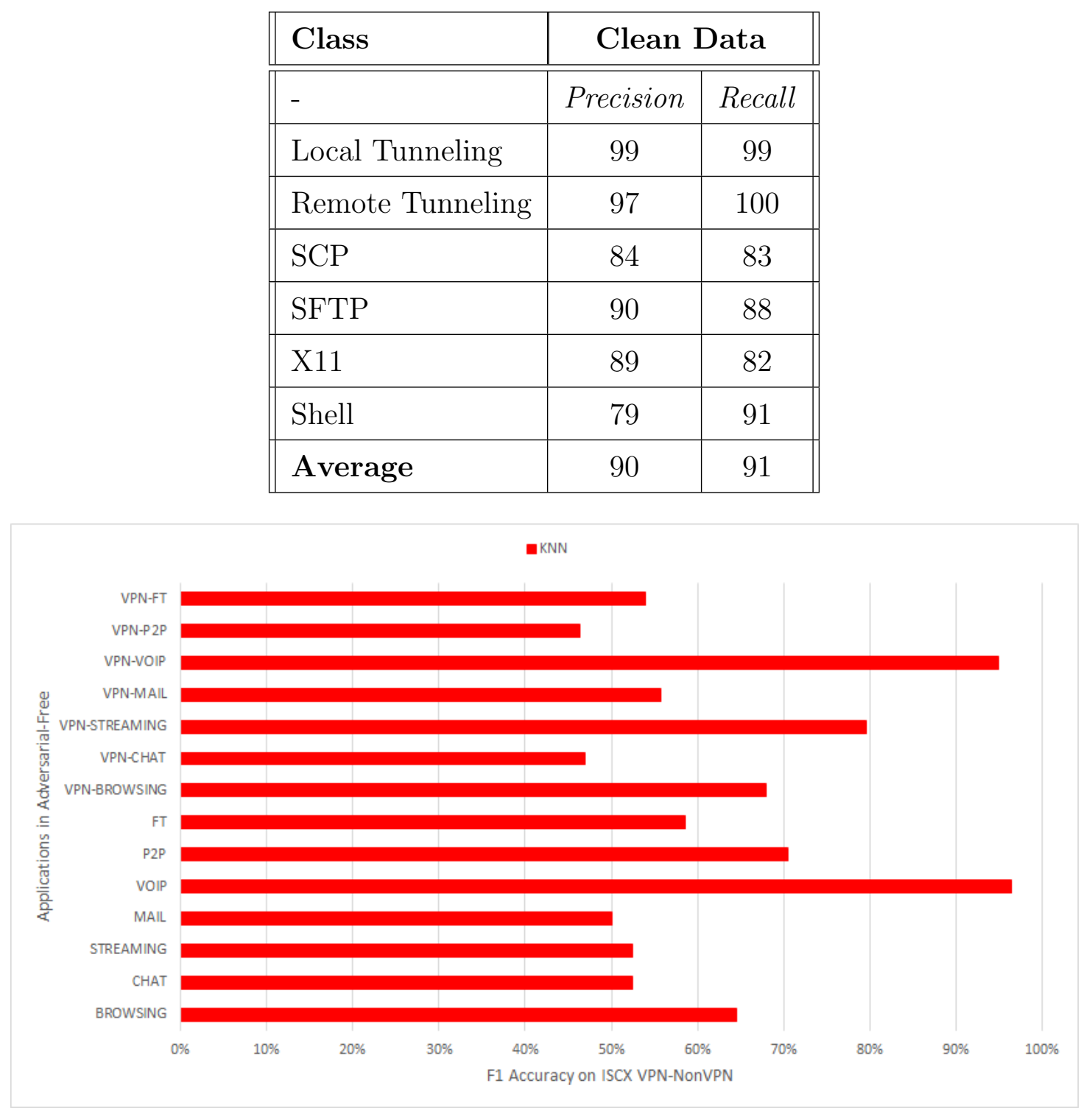

Figure 5.3: KNN models trained by Adversarial-free ISCX VPN-NonVPN dataset

NIMS categorical classification to help in comparing with the other model's performance. The result values are rounded to the nearest integer. The F1-Score accuracy for each application are stated in Figure 5.5 and 5.6.

For ISCX VPN-NonVPN, the ANN models prediction accuracy of Browsing, 


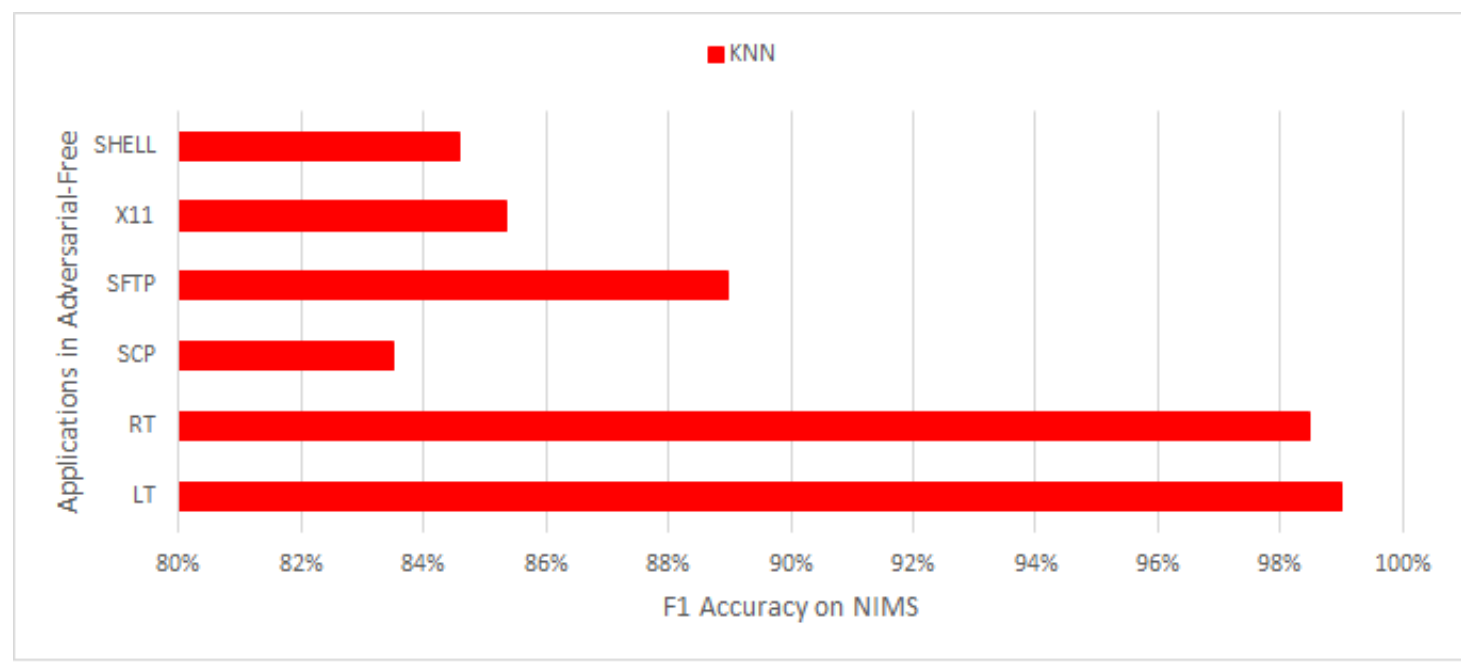

Figure 5.4: KNN models trained by Adversarial-free NIMS dataset

Streaming, and Mail in Non-VPN traffic was significantly improved in VPN traffic from $64 \%, 52 \%$, and $2 \%$ to $72 \%, 78 \%$ and $43 \%$, respectively, as shown in Figure 5.5 .

As shown in Figure 5.6, the F1-Score prediction of ANN models in NIMS was $97.5 \%$ and $98 \%$ for RT and X11, respectively. Unlike SFTP, which was lower in prediction by almost $7 \%$.

\subsubsection{Performance of CNN}

Table 5.8 and 5.9 illustrate the Precision and Recall metrics results initiated from the ISCX VPN-NonVPN and NIMS datasets in an adversarial-free environment to report CNN models' performance in acquiring encrypted traffic flow characterization and application identification. The result values are rounded to the nearest integer. The F1-Score accuracy for each application are presented in Figure 5.7 and 5.8.

Figure 5.7 propose the F1-Score prediction accuracy of CNN models in ISCX 
Table 5.6: Evaluation Metric Results (\%) of ANN models in adversarial-free environment for ISCX VPN-NonVPN

\begin{tabular}{||l|c|c||}
\hline Class & \multicolumn{2}{|c||}{ Clean Data } \\
\hline \hline- & Precision & Recall \\
\hline Browsing & 60 & 69 \\
\hline Chat & 53 & 61 \\
\hline Streaming & 50 & 55 \\
\hline Mail & 100 & 1 \\
\hline VoIP & 71 & 83 \\
\hline P2P & 50 & 68 \\
\hline File Transfer & 87 & 80 \\
\hline VPN-Browsing & 69 & 76 \\
\hline VPN-Chat & 60 & 37 \\
\hline VPN-Streaming & 67 & 92 \\
\hline VPN-Mail & 60 & 33 \\
\hline VPN-VoIP & 73 & 86 \\
\hline VPN-P2P & 59 & 57 \\
\hline VPN-File Transfer & 75 & 61 \\
\hline Average & 67 & 61 \\
\hline
\end{tabular}

VPN-NonVPN, where VoIP and P2P show high accuracy in Non-VPN traffic of $90 \%$ and $92 \%$ compared to VPN traffic which was $64 \%$ and $74 \%$, respectively. While, Mail shows a diversity in the accuracy prediction, which was low in Non-VPN traffic of $2 \%$ in contrast to VPN traffic of $73 \%$.

The prediction accuracy of CNN in NIMS for all applications was exceeding $95 \%$ where the lowest predicted application was SFTP of $95.5 \%$ and the highest predicted 
Table 5.7: Evaluation Metric Results (\%) of ANN models in adversarial-free environment for NIMS dataset

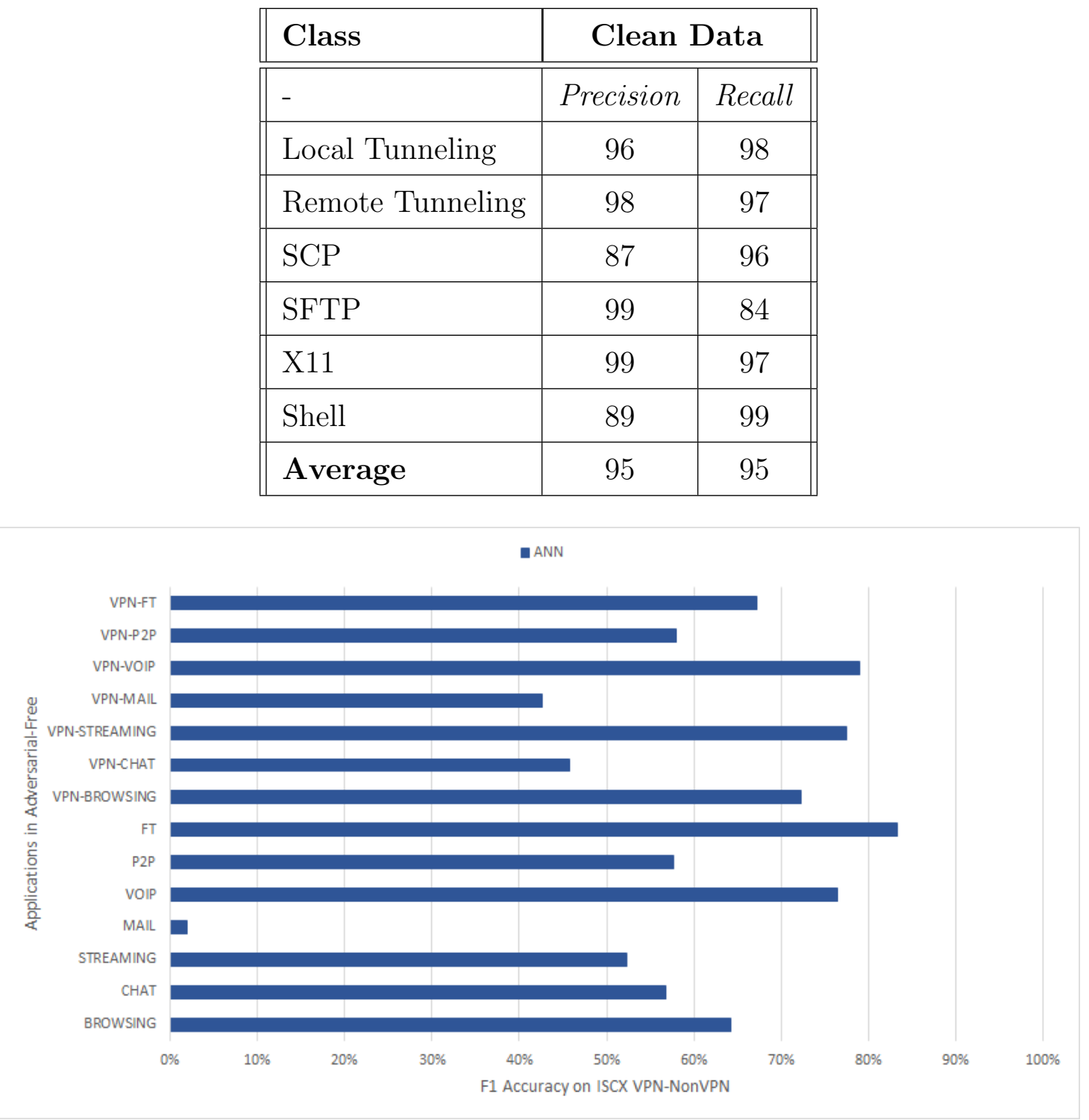

Figure 5.5: ANN models trained by Adversarial-free ISCX VPN-NonVPN dataset

applications with the same results of $97.5 \%$ are LT and SCP, as shown in Figure 5.8. 


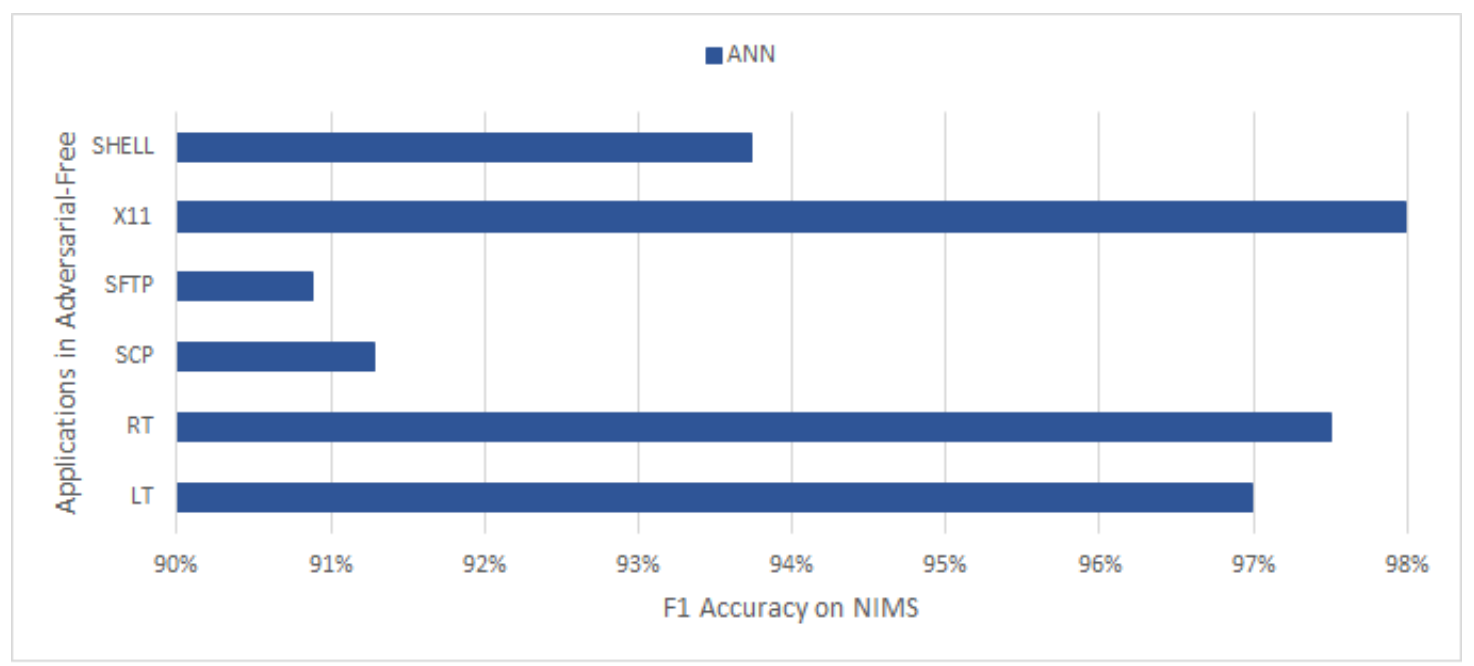

Figure 5.6: ANN models trained by Adversarial-free NIMS dataset

\subsubsection{Performance of RNN}

In this experiment, we will discuss the RNN model's abilities in identifying applications and learning encrypted traffic flow characterization on ISCX VPN-NonVPN and NIMS datasets. Table 5.10 and 5.11 analyze the performance metrics results in an adversarial-free for ISCX VPN-NonVPN and NIMS datasets. The result values are rounded to the nearest integer. Figure 5.9 and 5.10 show the F1-Score performance metrics results for each application.

As we can see in Figure 5.9, the Browsing, Mail, and FT got low F1-Score predicted accuracy in Non-VPN traffic of $44 \%, 1 \%$, and $53 \%$ in comparison to VPN traffic of $75 \%, 70 \%$, and $67 \%$ respectively.

Similar to CNN results in NIMS, Figure 5.10 shows that all application's prediction exceeds $95 \%$ and the top two applications have the same predicted results of $97.5 \%$ was X11 and Shell. 
Table 5.8: Evaluation Metric Results (\%) of CNN models in adversarial-free environment for ISCX VPN-NonVPN

\begin{tabular}{||l|c|c||}
\hline Class & \multicolumn{2}{|c||}{ Clean Data } \\
\hline \hline- & Precision & Recall \\
\hline Browsing & 52 & 40 \\
\hline Chat & 38 & 69 \\
\hline Streaming & 71 & 68 \\
\hline Mail & 12 & 1 \\
\hline VoIP & 91 & 89 \\
\hline P2P & 95 & 90 \\
\hline File Transfer & 40 & 83 \\
\hline VPN-Browsing & 81 & 73 \\
\hline VPN-Chat & 54 & 79 \\
\hline VPN-Streaming & 84 & 65 \\
\hline VPN-Mail & 81 & 67 \\
\hline VPN-VoIP & 82 & 53 \\
\hline VPN-P2P & 70 & 79 \\
\hline VPN-File Transfer & 83 & 80 \\
\hline Average & 67 & 67 \\
\hline
\end{tabular}

\subsection{Performance Comparison of ML and DL}

We train two machine learning, i.e., C4.5 and KNN, and three deep learning models, i.e., ANN, CNN, and RNN, on two adversarial-free datasets. Figure 5.11 shows the performance metrics of all models in the adversarial-free environment using the same top five selected features in Table 5.1.

If we look closer to each model in Figure 5.11 and evaluate its F1-Score accuracy in the ISCX VPN-NonVPN dataset, we can find that there is a slight improvement 
Table 5.9: Evaluation Metric Results (\%) of CNN models in adversarial-free environment for NIMS dataset

\begin{tabular}{||l|c|c||}
\hline Class & \multicolumn{2}{|c||}{ Clean Data } \\
\hline \hline- & Precision & Recall \\
\hline Local Tunneling & 97 & 98 \\
\hline Remote Tunneling & 98 & 95 \\
\hline SCP & 99 & 96 \\
\hline SFTP & 94 & 97 \\
\hline X11 & 98 & 96 \\
\hline Shell & 94 & 97 \\
\hline Average & 97 & 97 \\
\hline
\end{tabular}

in deep learning models performance in classifying encrypted traffic by $2-3 \%$, on average, in comparison to machine learning models. While in NIMS dataset, the deep learning performance was significantly better in classifying encrypted traffic by $5-7 \%$, on average, compared to machine learning models in the adversarial-free environment. 


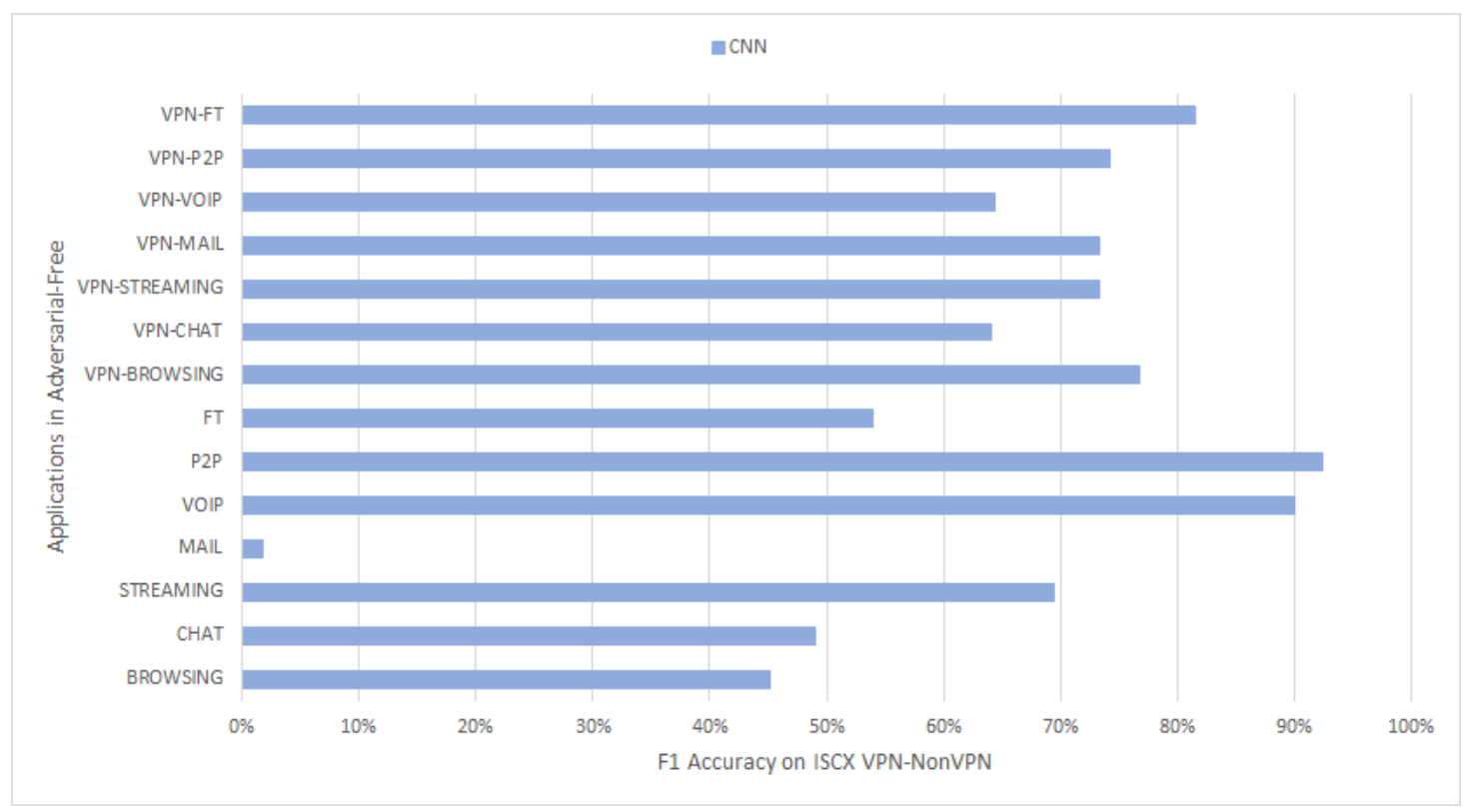

Figure 5.7: CNN models trained by Adversarial-free ISCX VPN-NonVPN dataset

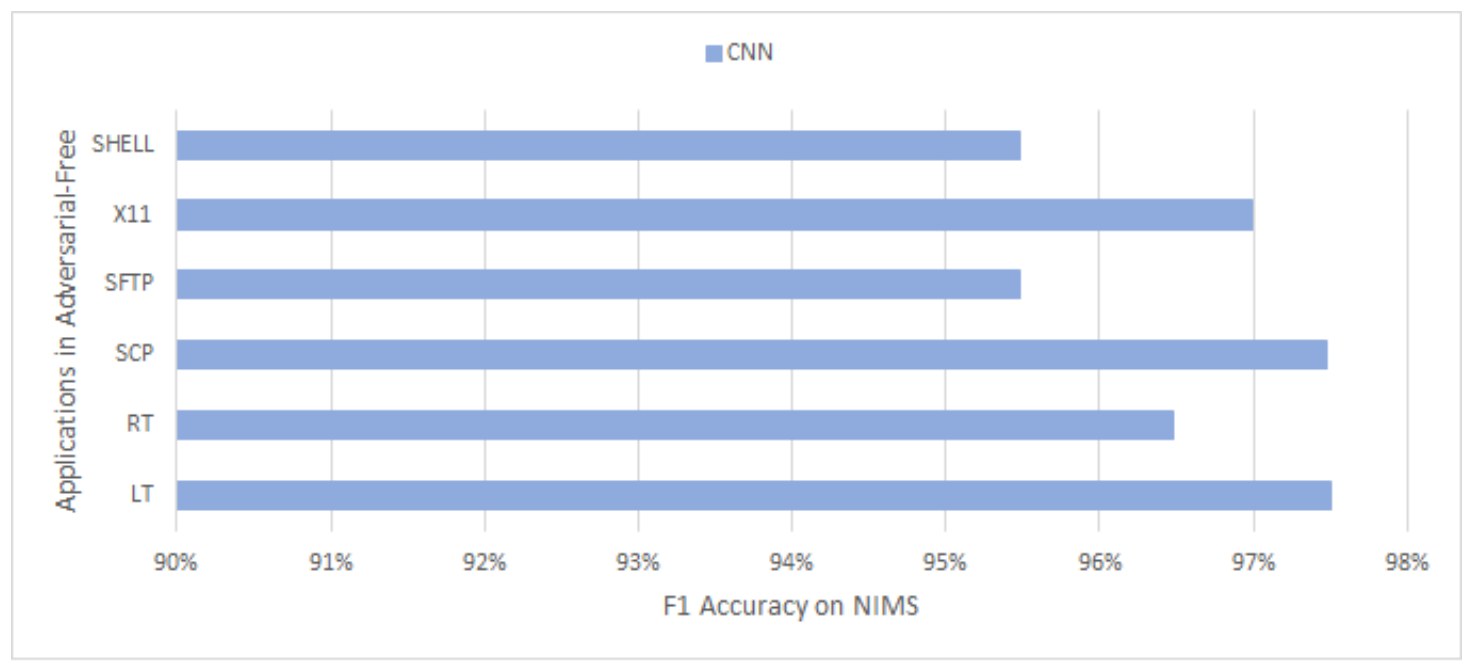

Figure 5.8: CNN models trained by Adversarial-free NIMS dataset 
Table 5.10: Evaluation Metric Results (\%) of RNN models in adversarial-free environment for ISCX VPN-NonVPN

\begin{tabular}{||l|c|c||}
\hline Class & \multicolumn{2}{|c||}{ Clean Data } \\
\hline \hline- & Precision & Recall \\
\hline Browsing & 41 & 47 \\
\hline Chat & 48 & 71 \\
\hline Streaming & 62 & 64 \\
\hline Mail & 1 & 1 \\
\hline VoIP & 95 & 98 \\
\hline P2P & 92 & 86 \\
\hline File Transfer & 38 & 85 \\
\hline VPN-Browsing & 77 & 73 \\
\hline VPN-Chat & 55 & 67 \\
\hline VPN-Streaming & 89 & 68 \\
\hline VPN-Mail & 80 & 63 \\
\hline VPN-VoIP & 87 & 57 \\
\hline VPN-P2P & 71 & 63 \\
\hline VPN-File Transfer & 84 & 83 \\
\hline Average & 66 & 66 \\
\hline
\end{tabular}


Table 5.11: Evaluation Metric Results (\%) of RNN models in adversarial-free environment for NIMS dataset

\begin{tabular}{||l|c|c||}
\hline Class & \multicolumn{2}{|c|}{ Clean Data } \\
\hline \hline- & Precision & Recall \\
\hline Local Tunneling & 100 & 94 \\
\hline Remote Tunneling & 97 & 96 \\
\hline SCP & 94 & 100 \\
\hline SFTP & 94 & 97 \\
\hline X11 & 99 & 96 \\
\hline Shell & 96 & 99 \\
\hline Average & 97 & 97 \\
\hline
\end{tabular}

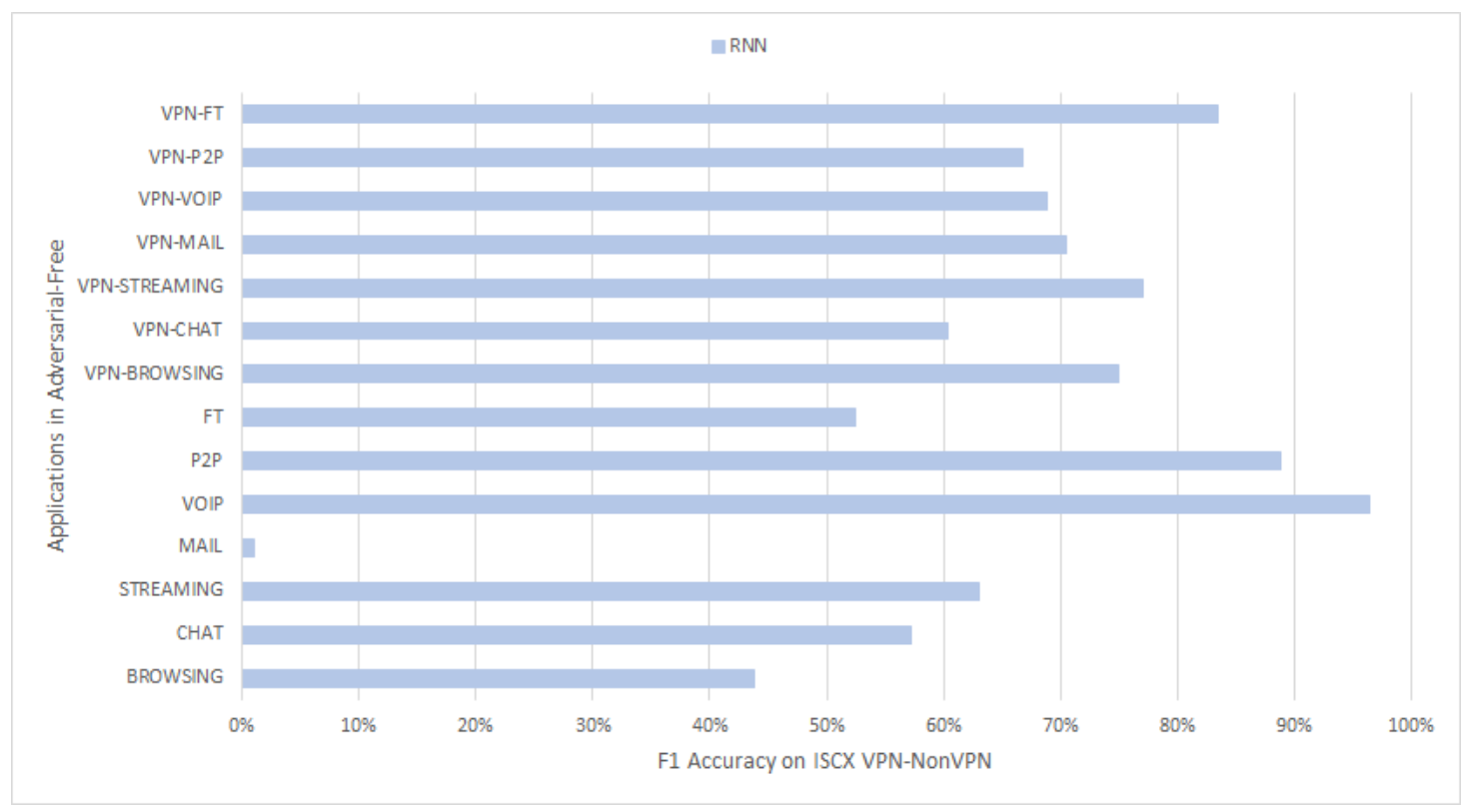

Figure 5.9: RNN models trained by Adversarial-free ISCX VPN-NonVPN dataset 


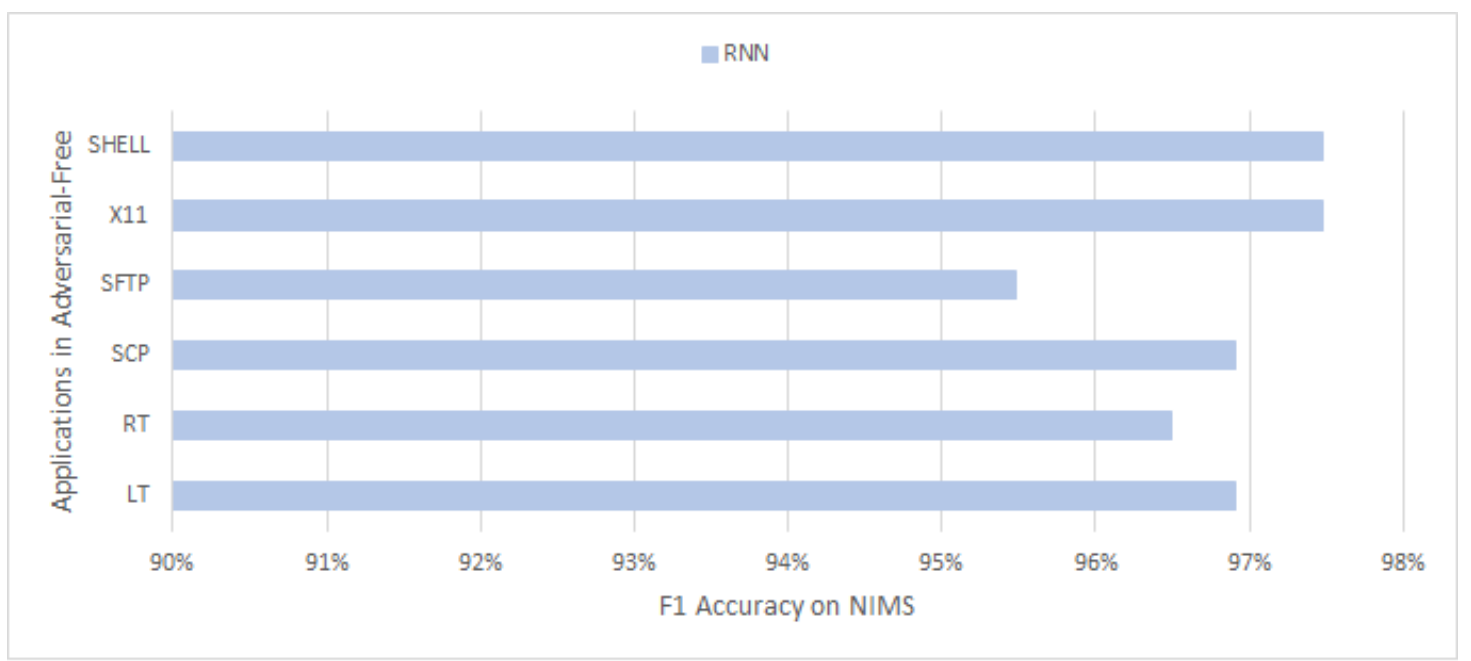

Figure 5.10: RNN models trained by Adversarial-free NIMS dataset

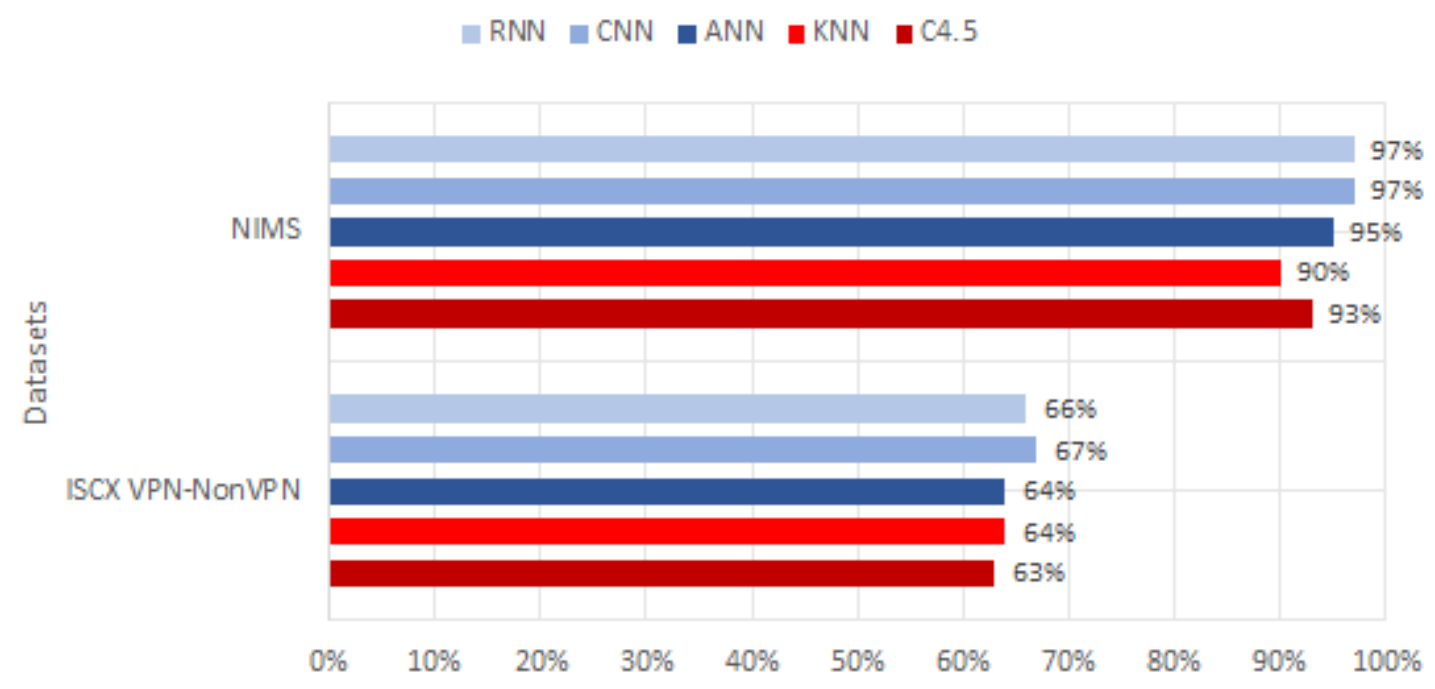

F1 Accuracy on Adv. Free Enviroment

Figure 5.11: Evaluation Metric Results (\%) of five models in adversarial-free environment. DL algorithms are shown in different shades of Blue. ML algorithms are shown in different shades of Red. 


\section{Chapter 6}

\section{ML and DL Resilience against Adversarial Evasion Attacks}

This chapter reports and evaluates the learning model's resilience against adversarial evasion attacks. Our experiment results illustrate the effectiveness of using different evasion attacks, i.e., PGD, ZOO, and DeepFool, against the machine and deep learning models: C4.5, KNN, ANN, CNN, and RNN on test data: ISCX VPN-NonVPN and NIMS. First, we start finding adversarial samples from the state-of-the-art adversarial attack methods with high perturbation to maximize the loss. Second, we evaluate the quality of the adversarial samples found on ISCX VPN-NonVPN and NIMS datasets against our five learning models. Finally, we evaluate the resilience

of the adversarially trained models against adversarial samples generated from the evasion attacks.

As we mentioned earlier, the proposed attacks are evasion attacks, where the attacker has access to our network and tries to predict the type of encrypted traffic and identify the application used, which misclassifies the model decision and targets the positive sample to be classified as a negative sample. We used the same adversarial threat models across all experiments done. We will notice sharp prediction 
accuracy fall among all learning models compared to that of the adversarial-free environments, which is expected due to the different ways of training these models with clean datasets or adversarial samples. Evaluation results are discussed in detail in the following sections.

\subsection{Evaluating ML Algorithms Resilience against Adversarial Attacks}

\subsubsection{Performance of C4.5}

We are exploring the C4.5 model's resilience against adversarial attacks on ISCX VPN-NonVPN and NIMS datasets. We can see that the C4.5 predicted accuracy was degraded by the adversarial samples generated from the two datasets using PGD, ZOO, and DeepFool adversarial evasion attacks in misleading encrypted traffic classifications.

For ISCX VPN-NonVPN and NIMS datasets, we show the C4.5 model's resilience per each application against the three adversarial attacks, as shown in Table 6.1 and 6.2. We calculate F1-Score using an average Precision and Recall to evaluate the model's performance and compare the results of the adversarial samples to that from the adversarial-free environment, as shown in Figure 6.1 and 6.2. As a reminder, we achieve F1-Score accuracy of $63 \%$ and $93 \%$ with no attack in Chapter 5 for ISCX VPN-NonVPN and NIMS, respectively.

The F1-Score accuracy of ISCX VPN-NonVPN is reduced from 53\%, 50\%, 98\%, and $57 \%$ to $0 \%$ for Streaming, Mail, VoIP, and VPN-Mail using PGD adversarial samples, respectively. Similarly, as illustrated in Figure 6.1 with ZOO and DeepFool adversarial samples, the accuracy of VoIP and VPN-Mail degraded to $0 \%$. 
For NIMS, the F1-Score accuracy is significantly reduced from 98\%, 85\%, and $97 \%$ to $3 \%, 5 \%$, and $6 \%$ for RT, SFTP, and X11 using DeepFool adversarial samples, respectively. At the same time, in Figure 6.2, accuracy decreased to 52\%, 16\%, and $43 \%$ using PGD adversarial samples, respectively.

We can conclude that the performance of C4.5 in the adversarial-free environment in NIMS was more resilient to the three adversarial attacks compared to ISCX VPNNonVPN. We also find that the DeepFool attack has the most influence on C4.5 models on NIMS dataset. Unlike the ISCX VPN-NonVPN dataset, where C4.5 models are less resilient to PGD evasion attacks. The ZOO attack achieves $12 \%$ and $23 \%$ as an average F1-Score accuracy on both datasets compared to the other two evasion attacks.

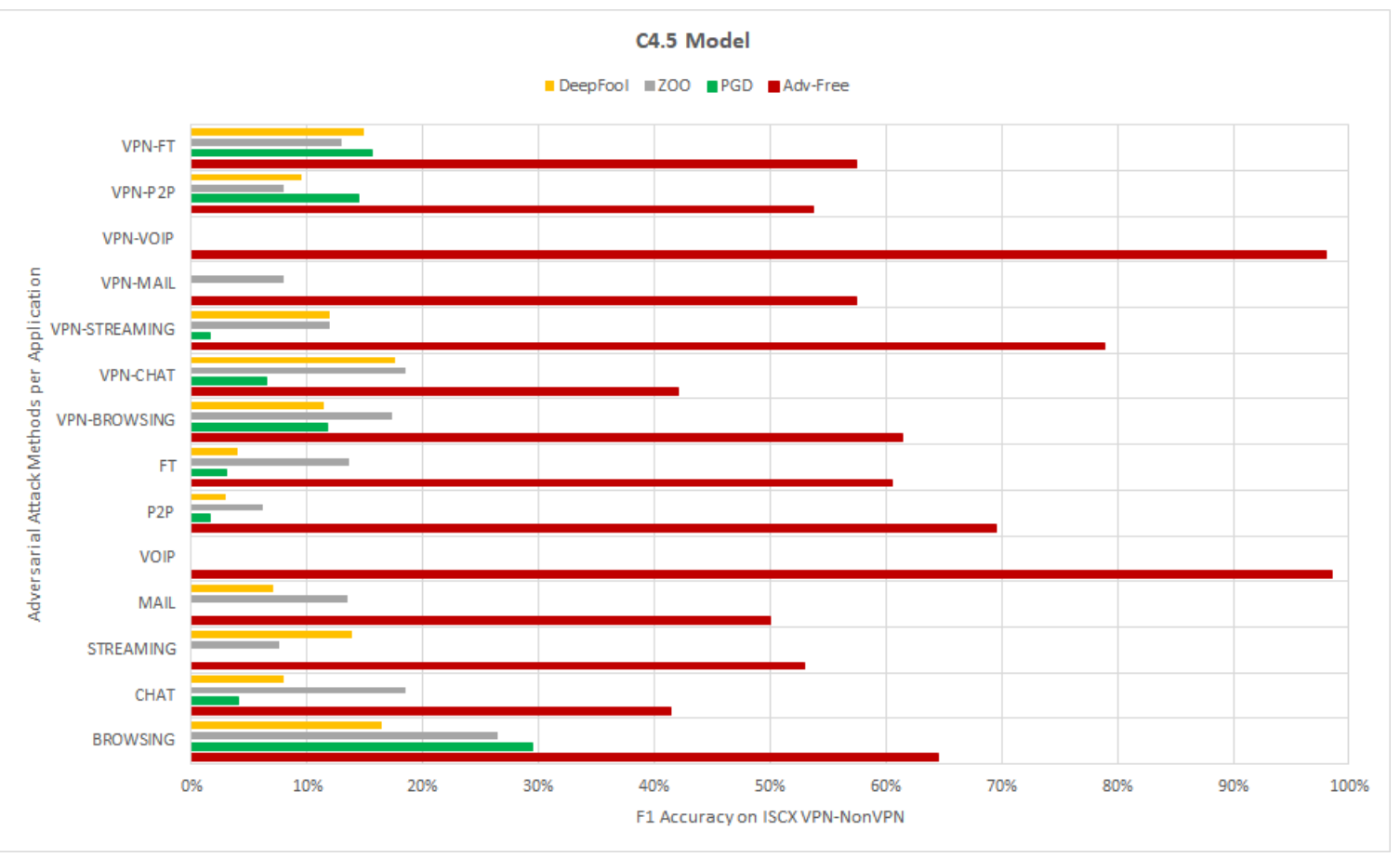

Figure 6.1: Effect of Adversarial Samples generated on C4.5 models trained by Adversarial-free ISCX VPN-NonVPN dataset 
Table 6.1: Evaluation Metric Results (\%) of $\mathrm{C} 4.5$ models in adversarial attack environment for ISCX VPN-NonVPN

\begin{tabular}{||l|c|c|c|c|c|c||}
\hline Class & \multicolumn{5}{c||}{ Attack Methods } \\
\hline \hline- & \multicolumn{2}{|c|}{ PGD } & \multicolumn{2}{c||}{ ZOO } & \multicolumn{2}{c||}{ DeepFool } \\
\hline- & Precision & Recall & Precision & Recall & Precision & Recall \\
\hline Browsing & 38 & 24 & 27 & 26 & 26 & 12 \\
\hline Chat & 4 & 4 & 22 & 16 & 19 & 5 \\
\hline Streaming & 0 & 0 & 5 & 16 & 13 & 15 \\
\hline Mail & 0 & 0 & 13 & 14 & 32 & 4 \\
\hline VoIP & 0 & 0 & 0 & 0 & 0 & 0 \\
\hline P2P & 4 & 1 & 13 & 4 & 3 & 3 \\
\hline File Transfer & 3 & 3 & 9 & 28 & 3 & 6 \\
\hline VPN-Browsing & 9 & 17 & 19 & 16 & 12 & 11 \\
\hline VPN-Chat & 18 & 4 & 16 & 22 & 27 & 13 \\
\hline VPN-Streaming & 1 & 4 & 10 & 15 & 15 & 10 \\
\hline VPN-Mail & 0 & 0 & 8 & 8 & 0 & 0 \\
\hline VPN-VoIP & 2 & 0 & 1 & 0 & 13 & 0 \\
\hline VPN-P2P & 14 & 15 & 8 & 8 & 10 & 9 \\
\hline VPN-File Transfer & 10 & 36 & 13 & 13 & 14 & 16 \\
\hline Average & 7 & 8 & 12 & 13 & 13 & 7 \\
\hline
\end{tabular}

\subsubsection{Performance of KNN}

As shown in Figure 6.3 and 6.4, the accuracy of the KNN model was degraded by the adversarial samples generated from ISCX VPN-NonVPN and NIMS datasets using the PGD, ZOO, and DeepFool adversarial evasion attacks in misleading encrypted traffic classifications.

For ISCX VPN-NonVPN and NIMS datasets, we show the KNN model's resilience per application against the adversarial attacks, as shown in Table 6.3 and 6.4. We 
Table 6.2: Evaluation Metric Results (\%) of $\mathrm{C} 4.5$ models in adversarial attack environment for NIMS dataset

\begin{tabular}{||l|c|c|c|c|c|c||}
\hline Class & \multicolumn{5}{c||}{ Attack Methods } \\
\hline \hline- & \multicolumn{2}{|c|}{ PGD } & \multicolumn{2}{c|}{ ZOO } & \multicolumn{2}{c||}{ DeepFool } \\
\hline- & Precision & Recall & Precision & Recall & Precision & Recall \\
\hline Local Tunneling & 25 & 9 & 9 & 6 & 16 & 28 \\
\hline Remote Tunneling & 49 & 55 & 41 & 28 & 10 & 2 \\
\hline SCP & 33 & 51 & 5 & 8 & 14 & 36 \\
\hline SFTP & 19 & 14 & 2 & 2 & 11 & 3 \\
\hline X11 & 40 & 46 & 40 & 47 & 8 & 5 \\
\hline Shell & 41 & 38 & 45 & 38 & 3 & 2 \\
\hline Average & 35 & 36 & 24 & 22 & 10 & 13 \\
\hline
\end{tabular}

calculate F1-Score using an average Precision and Recall to evaluate the model's performance and compare the results of the adversarial samples to that from the adversarial-free environment, as shown in Figure 6.3 and 6.4. As a reminder, we achieve F1-Score accuracy of $64 \%$ and $90 \%$ with no attack in Chapter 5 for ISCX VPN-NonVPN and NIMS, respectively.

The F1-Score accuracy of ISCX VPN-NonVPN is reduced from 50\%, 59\%, 68\%, and $80 \%$ to $3 \%, 5 \%, 4 \%$, and $2 \%$ for Mail, FT, VPN-Browsing, and VPN-Streaming using PGD adversarial samples, respectively. As shown in Figure 6.3 with ZOO and DeepFool adversarial samples, the F1-Score accuracy is degraded to $13 \%, 11 \%, 9 \%$, $11 \%$ and $7 \%, 9 \%, 16 \%, 15 \%$, respectively. We also noticed that ZOO and DeepFool strength are equal compared to PGD.

For NIMS, the F1-Score accuracy is reduced significantly from $98 \%$ and $85 \%$ to $6 \%$ and $2 \%$ for RT and X11 using DeepFool adversarial samples, respectively. F1 accuracy decreased to $34 \%, 34 \%$ and $20 \%, 36 \%$ using PGD and ZOO adversarial 


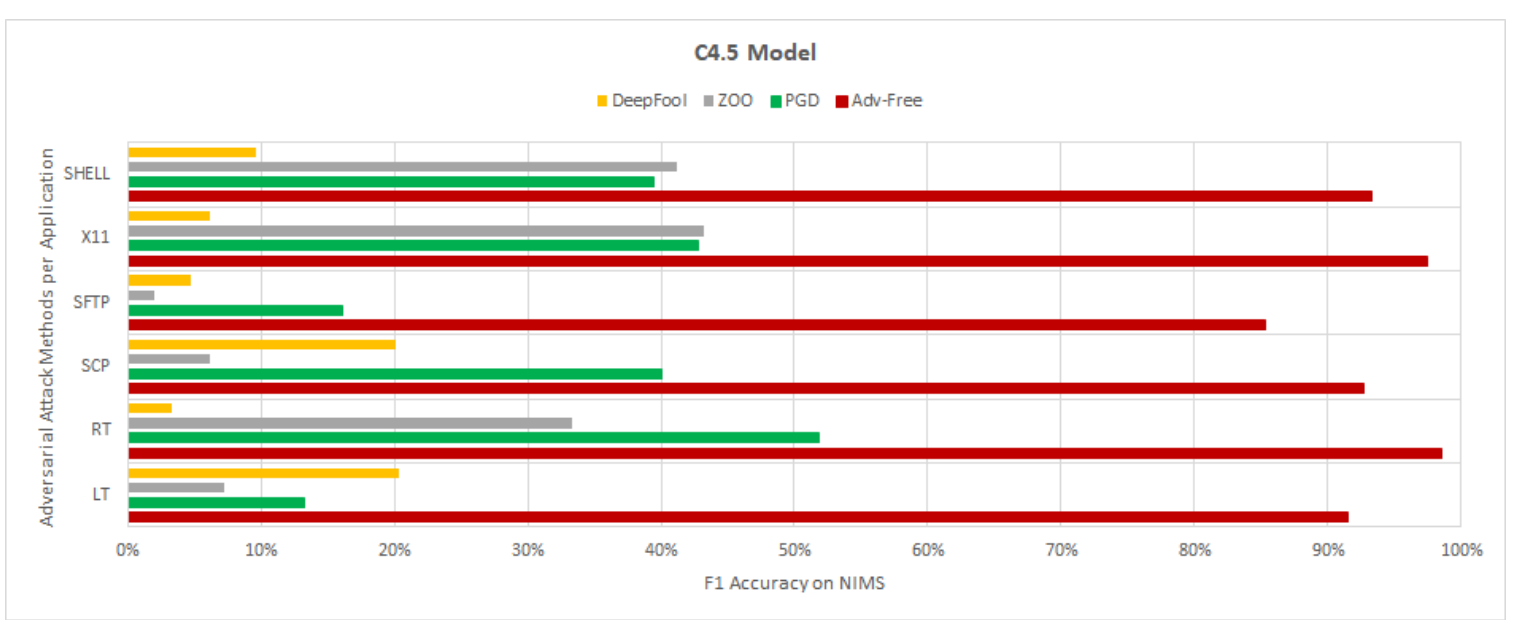

Figure 6.2: Effect of Adversarial Samples generated on C4.5 models trained by Adversarial-free NIMS dataset

samples, respectively, as illustrated in Figure 6.4.

Adversarial-free KNN trained on NIMS was more resilient to the three adversarial attacks than trained on ISCX VPN-NonVPN. We observe that KNN models on NIMS were less resilient to DeepFool attack and more resilient to PGD attack. In ISCX VPN-NonVPN, PGD has a higher impact comparing to ZOO and DeepFool attacks.

\subsection{Evaluating DL Algorithms Resilience against Adversarial Attacks}

\subsubsection{Performance of ANN}

It can be seen from Figure 6.5 and 6.6 that the ANN model accuracy was degraded by the adversarial samples generated from ISCX VPN-NonVPN and NIMS datasets using the PGD, ZOO, and DeepFool adversarial evasion attacks in misleading encrypted 
Table 6.3: Evaluation Metric Results (\%) of KNN models in adversarial attack environment for ISCX VPN-NonVPN

\begin{tabular}{||l|c|c|c|c|c|c||}
\hline Class & \multicolumn{5}{c||}{ Attack Methods } \\
\hline \hline- & \multicolumn{2}{|c|}{ PGD } & \multicolumn{2}{c||}{ ZOO } & \multicolumn{2}{c||}{ DeepFool } \\
\hline- & Precision & Recall & Precision & Recall & Precision & Recall \\
\hline Browsing & 18 & 20 & 36 & 27 & 19 & 21 \\
\hline Chat & 23 & 16 & 27 & 14 & 27 & 16 \\
\hline Streaming & 40 & 8 & 36 & 11 & 12 & 11 \\
\hline Mail & 4 & 2 & 17 & 10 & 37 & 4 \\
\hline VoIP & 0 & 1 & 0 & 2 & 0 & 0 \\
\hline P2P & 41 & 12 & 21 & 12 & 8 & 7 \\
\hline File Transfer & 4 & 8 & 7 & 26 & 7 & 13 \\
\hline VPN-Browsing & 15 & 2 & 19 & 6 & 13 & 20 \\
\hline VPN-Chat & 7 & 6 & 12 & 25 & 16 & 11 \\
\hline VPN-Streaming & 11 & 1 & 8 & 17 & 13 & 18 \\
\hline VPN-Mail & 0 & 0 & 8 & 4 & 0 & 0 \\
\hline VPN-VoIP & 0 & 0 & 0 & 0 & 18 & 2 \\
\hline VPN-P2P & 14 & 5 & 7 & 4 & 9 & 4 \\
\hline VPN-File Transfer & 9 & 22 & 6 & 5 & 9 & 14 \\
\hline Average & 13 & 7 & 15 & 12 & 13 & 10 \\
\hline
\end{tabular}

traffic classifications.

For ISCX VPN-NonVPN and NIMS datasets, we show the ANN model's resilience per each application against the three adversarial attack's Precision and Recall, as shown in Table 6.5 and 6.6. We calculate F1-Score using an average Precision and Recall to evaluate the model's performance and compare the results of the adversarial samples to that from the adversarial-free environment, as shown in Figure 6.5 and 6.6. As a reminder, we achieve F1-Score accuracy of $64 \%$ and $95 \%$ with no attack in Chapter 5 for ISCX VPN-NonVPN and NIMS, respectively. 
Table 6.4: Evaluation Metric Results (\%) of KNN models in adversarial attack environment for NIMS dataset

\begin{tabular}{||l|c|c|c|c|c|c||}
\hline \multirow{2}{*}{ Class } & \multicolumn{5}{c||}{ Attack Methods } \\
\hline \hline - & \multicolumn{2}{|c|}{ PGD } & \multicolumn{2}{c|}{ ZOO } & \multicolumn{2}{c||}{ DeepFool } \\
\hline - & Precision & Recall & Precision & Recall & Precision & Recall \\
\hline Local Tunneling & 37 & 15 & 15 & 18 & 19 & 7 \\
\hline Remote Tunneling & 37 & 31 & 22 & 19 & 4 & 12 \\
\hline SCP & 32 & 53 & 17 & 23 & 31 & 20 \\
\hline SFTP & 28 & 15 & 23 & 26 & 16 & 11 \\
\hline X11 & 26 & 50 & 39 & 33 & 13 & 1 \\
\hline Shell & 23 & 14 & 15 & 9 & 19 & 17 \\
\hline Average & 31 & 30 & 22 & 21 & 17 & 11 \\
\hline
\end{tabular}

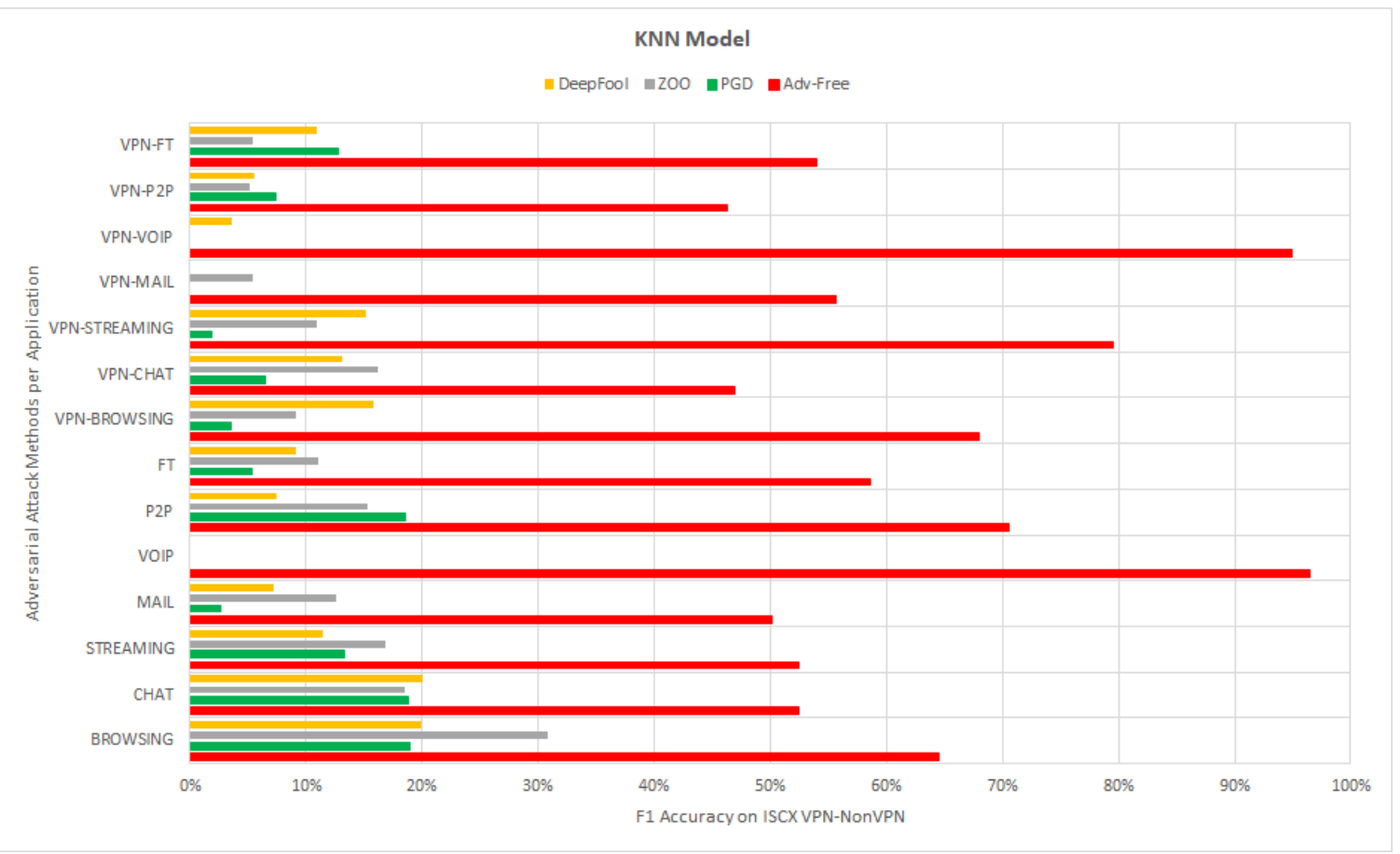

Figure 6.3: Effect of Adversarial Samples generated on KNN models trained by Adversarial-free ISCX VPN-NonVPN dataset 


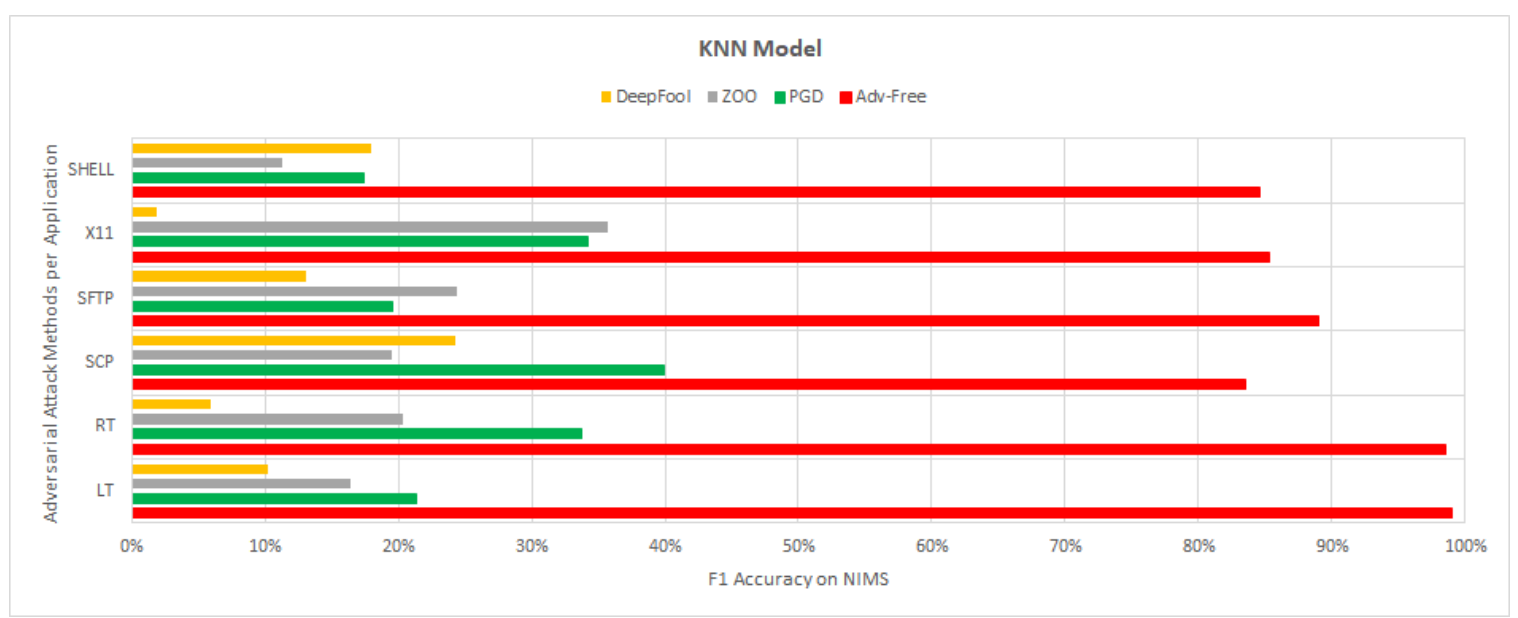

Figure 6.4: Effect of Adversarial Samples generated on KNN models trained by Adversarial-free NIMS dataset

As shown in Figure 6.5, the F1-Score accuracy of ANN in ISCX VPN-NonVPN is reduced by almost $70 \%$ from the original accuracy. We also noticed that PGD, ZOO, and DeepFool consider having the same effect on ANN. DeepFool attack has the most considerable influence on the ANN on ISCX VPN-NonVPN.

For NIMS, the F1-Score accuracy is outstandingly reduced from 97\%, 91\%, and $98 \%$ to $0 \%, 3 \%$, and $2 \%$ for RT, SFTP, and X11 using DeepFool adversarial attack, respectively. We can notice that the PGD and ZOO attacks decreased the F1- Score accuracy to $49 \%, 22 \%, 35 \%$ and $23 \%, 20 \%, 52 \%$, respectively, as illustrated in Figure 6.6 .

We can conclude that the performance of ANN in the adversarial-free environment in NIMS was more resilient to the three adversarial attacks compared to ISCX VPNNonVPN. We also find that the DeepFool attack has the most influence on ANN models on both datasets. 
Table 6.5: Evaluation Metric Results (\%) of ANN models in adversarial attack environment for ISCX VPN-NonVPN

\begin{tabular}{||l|c|c|c|c|c|c||}
\hline Class & \multicolumn{5}{c||}{ Attack Methods } \\
\hline \hline- & \multicolumn{2}{|c|}{ PGD } & \multicolumn{2}{c||}{ ZOO } & \multicolumn{2}{c||}{ DeepFool } \\
\hline- & Precision & Recall & Precision & Recall & Precision & Recall \\
\hline Browsing & 34 & 8 & 17 & 20 & 14 & 6 \\
\hline Chat & 6 & 10 & 28 & 1 & 11 & 1 \\
\hline Streaming & 30 & 54 & 0 & 0 & 5 & 2 \\
\hline Mail & 0 & 0 & 0 & 0 & 0 & 0 \\
\hline VoIP & 1 & 1 & 41 & 13 & 2 & 0 \\
\hline P2P & 0 & 0 & 25 & 15 & 0 & 0 \\
\hline File Transfer & 14 & 15 & 5 & 30 & 3 & 17 \\
\hline VPN-Browsing & 15 & 5 & 47 & 3 & 3 & 4 \\
\hline VPN-Chat & 11 & 0 & 0 & 0 & 14 & 0 \\
\hline VPN-Streaming & 40 & 2 & 79 & 20 & 10 & 4 \\
\hline VPN-Mail & 0 & 0 & 0 & 0 & 4 & 1 \\
\hline VPN-VoIP & 25 & 19 & 0 & 0 & 19 & 22 \\
\hline VPN-P2P & 0 & 0 & 42 & 0 & 25 & 31 \\
\hline VPN-File Transfer & 3 & 2 & 20 & 21 & 19 & 16 \\
\hline Average & 13 & 8 & 22 & 9 & 9 & 7 \\
\hline
\end{tabular}


Table 6.6: Evaluation Metric Results (\%) of ANN models in adversarial attack environment for NIMS dataset

\begin{tabular}{||l|c|c|c|c|c|c||}
\hline Class & \multicolumn{5}{c||}{ Attack Methods } \\
\hline \hline- & \multicolumn{2}{|c|}{ PGD } & \multicolumn{2}{c||}{ ZOO } & \multicolumn{2}{c||}{ DeepFool } \\
\hline- & Precision & Recall & Precision & Recall & Precision & Recall \\
\hline Local Tunneling & 33 & 15 & 47 & 20 & 15 & 23 \\
\hline Remote Tunneling & 49 & 49 & 31 & 18 & 7 & 0 \\
\hline SCP & 39 & 45 & 9 & 13 & 9 & 23 \\
\hline SFTP & 27 & 19 & 23 & 17 & 3 & 3 \\
\hline X11 & 39 & 32 & 51 & 53 & 3 & 1 \\
\hline Shell & 42 & 47 & 21 & 6 & 2 & 10 \\
\hline Average & 38 & 35 & 30 & 21 & 7 & 10 \\
\hline
\end{tabular}

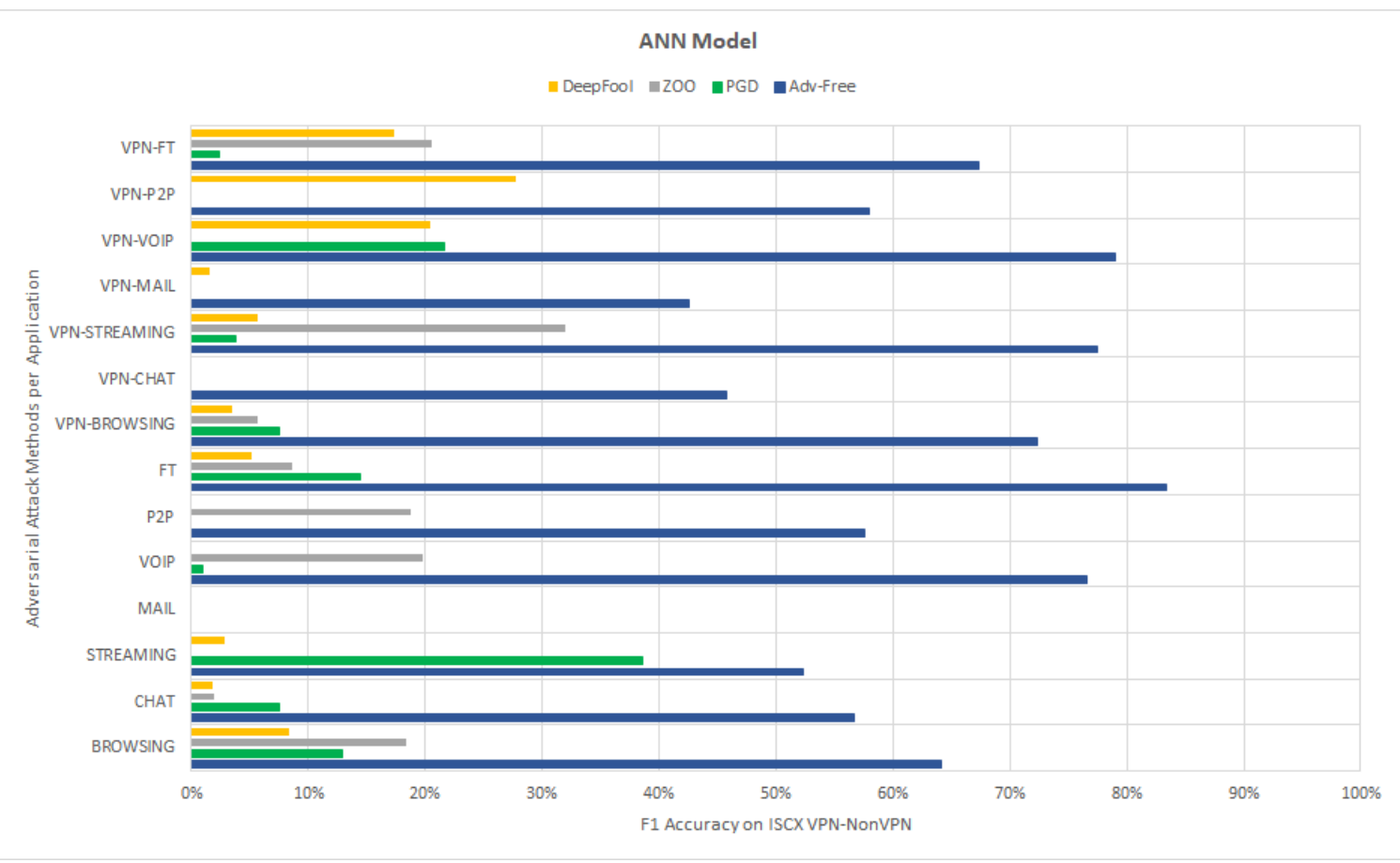

Figure 6.5: Effect of Adversarial Samples generated on ANN models trained by Adversarial-free ISCX VPN-NonVPN dataset 


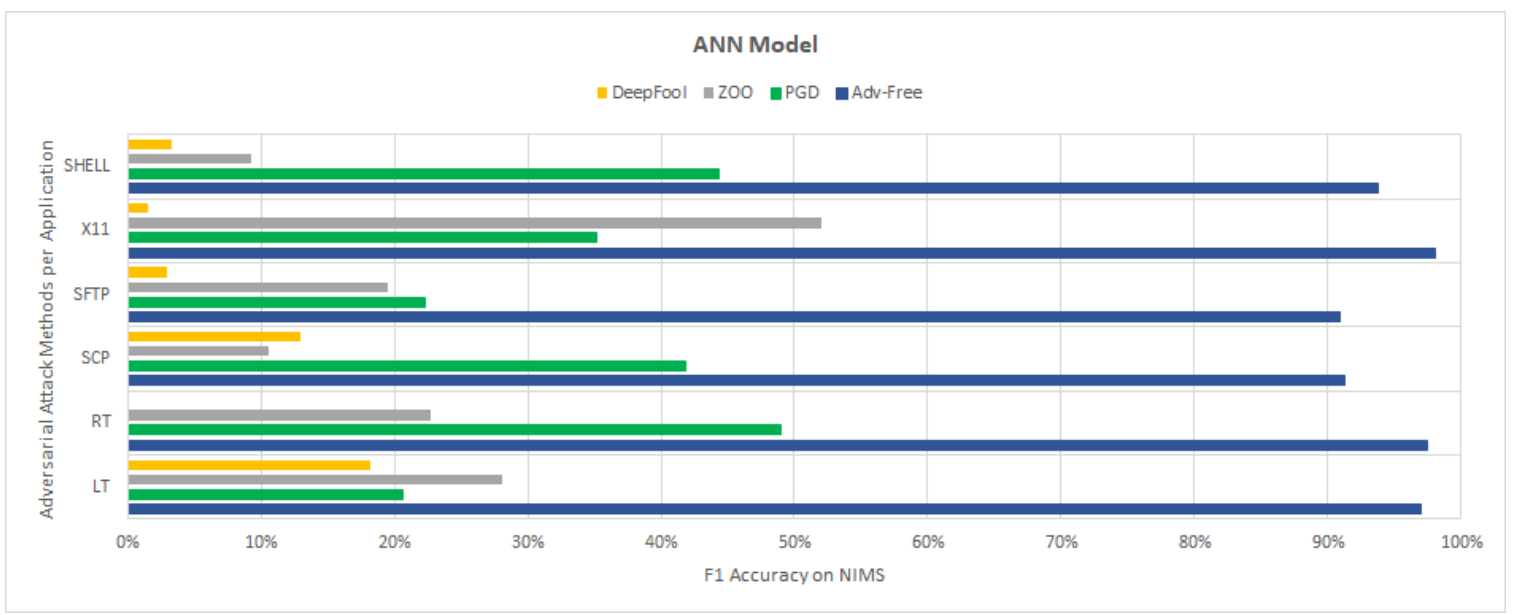

Figure 6.6: Effect of Adversarial Samples generated on ANN models trained by Adversarial-free NIMS dataset

\subsubsection{Performance of CNN}

We can demonstrate that the CNN model accuracy on Figure 6.7 and Figure 6.8 were also degraded by the adversarial samples generated from ISCX VPN-NonVPN and NIMS datasets using the PGD, ZOO, and DeepFool adversarial evasion attacks in misleading encrypted traffic classifications.

For ISCX VPN-NonVPN and NIMS datasets, we show the CNN model's resilience per each application against the three adversarial attack's Precision and Recall, as shown in Table 6.7 and 6.8. We calculate F1-Score using an average Precision and Recall to evaluate the model's performance and compare the results of the adversarial samples to that from the adversarial-free environment, as shown in Figure 6.7 and 6.8. As a reminder, we achieve F1-Score accuracy of $67 \%$ and $97 \%$ with no attack in Chapter 5 for ISCX VPN-NonVPN and NIMS, respectively.

The F1-Score accuracy of ISCX VPN-NonVPN is reduced from $69 \%, 92 \%, 64 \%$, and $81 \%$ to $0 \%$ for Streaming, P2P, VPN-VoIP, and VPN-FT using DeepFool adversarial samples, respectively. Similarly, as illustrated in Figure 6.7 with PGD and ZOO adversarial samples, the accuracy of CNN degraded to $23 \%, 30 \%, 7 \%$ and $40 \%$, 
$8 \%, 10 \%$, respectively.

For NIMS, the F1-Score accuracy is significantly reduced from $96 \%$ and $97 \%$ to $5 \%$ and $0 \%$ for RT and X11, using DeepFool adversarial samples, respectively. At the same time, in Figure 6.8, accuracy decreased to $42 \%, 36 \%$ and 20\%, 50\% using PGD and ZOO adversarial samples, respectively.

We can conclude that the performance of $\mathrm{CNN}$ in the adversarial-free environment in NIMS was more resilient to the three adversarial attacks compared to ISCX VPNNonVPN. We also find that the CNN models are less resilient to the DeepFool attack on both datasets. The resilience of CNN against PGD and ZOO was almost the same on both datasets compared to the DeepFool evasion attack.

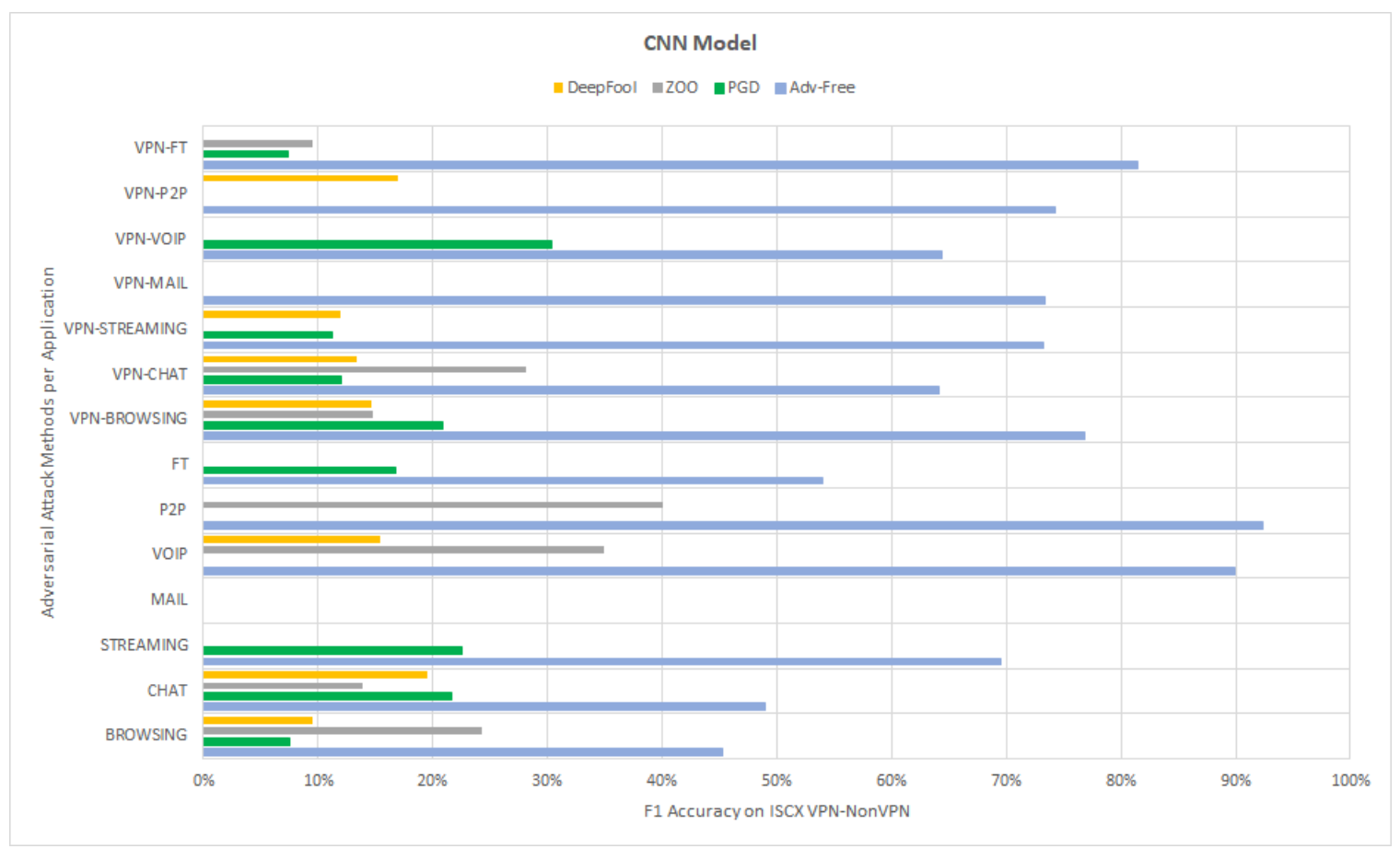

Figure 6.7: Effect of Adversarial Samples generated on CNN models trained by Adversarial-free ISCX VPN-NonVPN dataset 
Table 6.7: Evaluation Metric Results (\%) of CNN models in adversarial attack environment for ISCX VPN-NonVPN

\begin{tabular}{||l|c|c|c|c|c|c||}
\hline Class & \multicolumn{5}{c||}{ Attack Methods } \\
\hline \hline- & \multicolumn{2}{|c|}{ PGD } & \multicolumn{2}{c||}{ ZOO } & \multicolumn{2}{c||}{ DeepFool } \\
\hline- & Precision & Recall & Precision & Recall & Precision & Recall \\
\hline Browsing & 6 & 10 & 31 & 20 & 10 & 9 \\
\hline Chat & 18 & 27 & 19 & 11 & 28 & 15 \\
\hline Streaming & 16 & 38 & 0 & 0 & 0 & 0 \\
\hline Mail & 0 & 0 & 0 & 0 & 0 & 0 \\
\hline VoIP & 0 & 9 & 40 & 31 & 34 & 10 \\
\hline P2P & 0 & 0 & 26 & 87 & 20 & 0 \\
\hline File Transfer & 15 & 19 & 0 & 0 & 0 & 0 \\
\hline VPN-Browsing & 27 & 17 & 42 & 9 & 27 & 10 \\
\hline VPN-Chat & 15 & 10 & 22 & 39 & 11 & 17 \\
\hline VPN-Streaming & 19 & 8 & 0 & 0 & 18 & 9 \\
\hline VPN-Mail & 0 & 0 & 0 & 0 & 0 & 0 \\
\hline VPN-VoIP & 32 & 29 & 0 & 8 & 0 & 0 \\
\hline VPN-P2P & 0 & 0 & 35 & 0 & 18 & 16 \\
\hline VPN-File Transfer & 14 & 5 & 15 & 7 & 0 & 24 \\
\hline Average & 12 & 12 & 16 & 15 & 12 & 8 \\
\hline
\end{tabular}


Table 6.8: Evaluation Metric Results (\%) of CNN models in adversarial attack environment for NIMS dataset

\begin{tabular}{||l|c|c|c|c|c|c||}
\hline Class & \multicolumn{5}{c||}{ Attack Methods } \\
\hline \hline- & \multicolumn{2}{|c|}{ PGD } & \multicolumn{2}{c|}{ ZOO } & \multicolumn{2}{c||}{ DeepFool } \\
\hline- & Precision & Recall & Precision & Recall & Precision & Recall \\
\hline Local Tunneling & 32 & 40 & 40 & 51 & 10 & 16 \\
\hline Remote Tunneling & 51 & 35 & 23 & 17 & 4 & 8 \\
\hline SCP & 31 & 18 & 22 & 18 & 19 & 9 \\
\hline SFTP & 30 & 40 & 10 & 9 & 12 & 21 \\
\hline X11 & 35 & 38 & 51 & 50 & 0 & 0 \\
\hline Shell & 73 & 45 & 28 & 26 & 18 & 21 \\
\hline Average & 42 & 36 & 29 & 29 & 11 & 13 \\
\hline
\end{tabular}

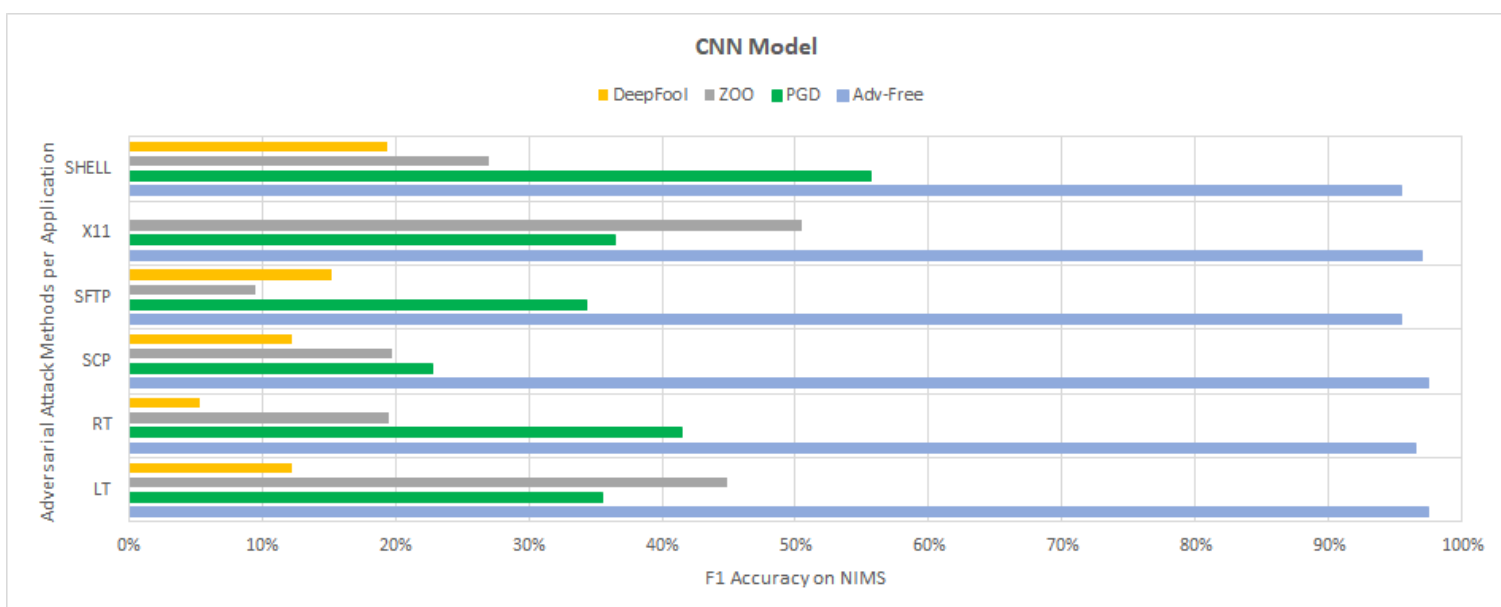

Figure 6.8: Effect of Adversarial Samples generated on CNN models trained by Adversarial-free NIMS dataset

\subsubsection{Performance of RNN}

Consistently with the result of the ISCX VPN-NonVPN and NIMS on CNN and ANN experiments, it can be seen from Figure 6.9 and Figure 6.10 that the RNN models was degraded by the adversarial samples generated from ISCX VPN-NonVPN and 
NIMS datasets using the PGD, ZOO, and DeepFool adversarial evasion attacks in misleading encrypted traffic classifications.

For ISCX VPN-NonVPN and NIMS datasets, we show the RNN model's resilience per each application against the three adversarial attack's Precision and Recall, as shown in Table 6.9 and 6.10. We use an average Precision and Recall to calculate F1Score to evaluate the model's performance and compare the results of the adversarial samples to that from the adversarial-free environment, as shown in Figure 6.9 and 6.10. As a reminder, we achieve F1-Score accuracy of $66 \%$ and $97 \%$ with no attack in Chapter 5 for ISCX VPN-NonVPN and NIMS, respectively.

As shown in Figure 6.9, the F1-Score accuracy of RNN in ISCX VPN-NonVPN is reduced by almost $80 \%$ from the original accuracy. We also noticed that PGD, ZOO, and DeepFool consider having the same effect on RNN. DeepFool attack has the most considerable influence on the RNN on ISCX VPN-NonVPN.

For NIMS, the F1-Score accuracy is reduced from $97 \%, 96 \%$, and $97 \%$ to $8 \%, 4 \%$, and $3 \%$ for LT, RT, and X11 using DeepFool adversarial samples, respectively. At the same time, in Figure 6.10, accuracy decreased significantly to $32 \%, 41 \%, 35 \%$ and 20\%, 28\%, 51\% using PGD and ZOO adversarial samples, respectively.

We can conclude that the performance of RNN in the adversarial-free environment in NIMS was more resilient to the three adversarial attacks compared to ISCX VPNNonVPN. We also find that the DeepFool attack has the most influence on RNN models on both datasets. The resilience of RNN was less against ZOO in NIMS compared to PGD attacks. The effect of the PGD and ZOO attacks was equal, on average, on ISCX VPN-NonVPN. 
Table 6.9: Evaluation Metric Results (\%) of RNN models in adversarial attack environment for ISCX VPN-NonVPN

\begin{tabular}{||l|c|c|c|c|c|c||}
\hline Class & \multicolumn{5}{c||}{ Attack Methods } \\
\hline \hline- & \multicolumn{2}{|c|}{ PGD } & \multicolumn{2}{c||}{ ZOO } & \multicolumn{2}{c||}{ DeepFool } \\
\hline- & Precision & Recall & Precision & Recall & Precision & Recall \\
\hline Browsing & 7 & 16 & 24 & 19 & 12 & 6 \\
\hline Chat & 27 & 17 & 23 & 10 & 23 & 5 \\
\hline Streaming & 19 & 43 & 0 & 0 & 0 & 0 \\
\hline Mail & 0 & 0 & 0 & 0 & 0 & 0 \\
\hline VoIP & 0 & 1 & 52 & 10 & 53 & 12 \\
\hline P2P & 0 & 0 & 16 & 79 & 25 & 1 \\
\hline File Transfer & 10 & 11 & 0 & 0 & 0 & 10 \\
\hline VPN-Browsing & 18 & 14 & 21 & 18 & 35 & 13 \\
\hline VPN-Chat & 18 & 13 & 24 & 28 & 17 & 7 \\
\hline VPN-Streaming & 20 & 8 & 0 & 0 & 16 & 14 \\
\hline VPN-Mail & 0 & 0 & 0 & 0 & 0 & 0 \\
\hline VPN-VoIP & 33 & 31 & 9 & 0 & 0 & 1 \\
\hline VPN-P2P & 0 & 0 & 32 & 4 & 10 & 4 \\
\hline VPN-File Transfer & 11 & 6 & 25 & 18 & 15 & 25 \\
\hline Average & 12 & 11 & 16 & 13 & 15 & 7 \\
\hline
\end{tabular}

\subsection{Performance Comparison of $\mathrm{ML}$ and $\mathrm{DL}$ in}

\section{Adversarial Environment}

We evaluate the resilience of the trained models in classifying encrypted traffic against the crafted adversarial samples (using PGD, ZOO, and DeepFool evasion attacks). In this experiment, we build the learning models with different architecture, and we preprocess the benchmark datasets: ISCX VPN-NonVPN and NIMS using the 
Table 6.10: Evaluation Metric Results (\%) of RNN models in adversarial attack environment for NIMS dataset

\begin{tabular}{||l|c|c|c|c|c|c||}
\hline Class & \multicolumn{5}{c||}{ Attack Methods } \\
\hline \hline- & \multicolumn{2}{|c|}{ PGD } & \multicolumn{2}{c|}{ ZOO } & \multicolumn{2}{c||}{ DeepFool } \\
\hline- & Precision & Recall & Precision & Recall & Precision & Recall \\
\hline Local Tunneling & 27 & 40 & 25 & 17 & 17 & 5 \\
\hline Remote Tunneling & 43 & 40 & 49 & 20 & 14 & 2 \\
\hline SCP & 23 & 12 & 12 & 22 & 23 & 7 \\
\hline SFTP & 35 & 42 & 18 & 15 & 10 & 28 \\
\hline X11 & 35 & 35 & 46 & 57 & 4 & 3 \\
\hline Shell & 64 & 56 & 31 & 18 & 9 & 11 \\
\hline Average & 38 & 38 & 30 & 25 & 13 & 9 \\
\hline
\end{tabular}

same top features. Figure 6.11 and 6.12 compare the effect of the adversarial attack methods against the learning algorithms. From Figure 6.11 and 6.12, we can see that machine learning and deep learning model accuracy were degraded significantly by the adversarial samples generated from the two datasets using the PGD, ZOO, and DeepFool evasion attacks.

In the ISCX VPN-NonVPN dataset in Figure 6.11, we can conclude that, on average, deep learning models were more resilient in classifying encrypted traffic to ZOO and PGD attacks compared to machine learning models. We also find that the resilience of deep learning was lower against the DeepFool attack than other attacks.

In NIMS dataset, deep learning performance shows more resilience in classifying encrypted traffic against ZOO and PGD adversarial samples compared to machine learning, as shown in Figure 6.12. Unlike DeepFool attack where deep learning was 


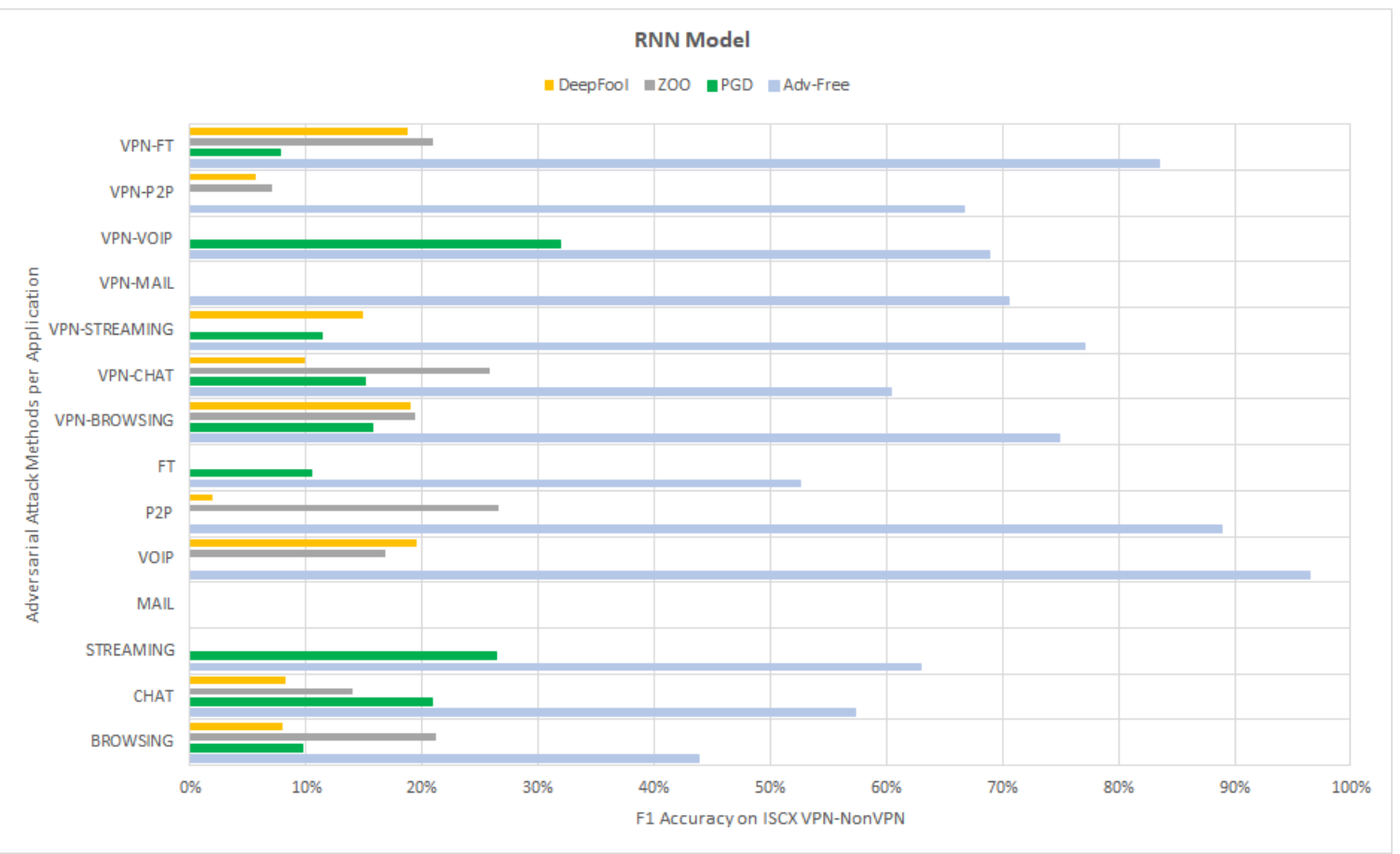

Figure 6.9: Effect of Adversarial Samples generated on RNN models trained by Adversarial-free ISCX VPN-NonVPN dataset

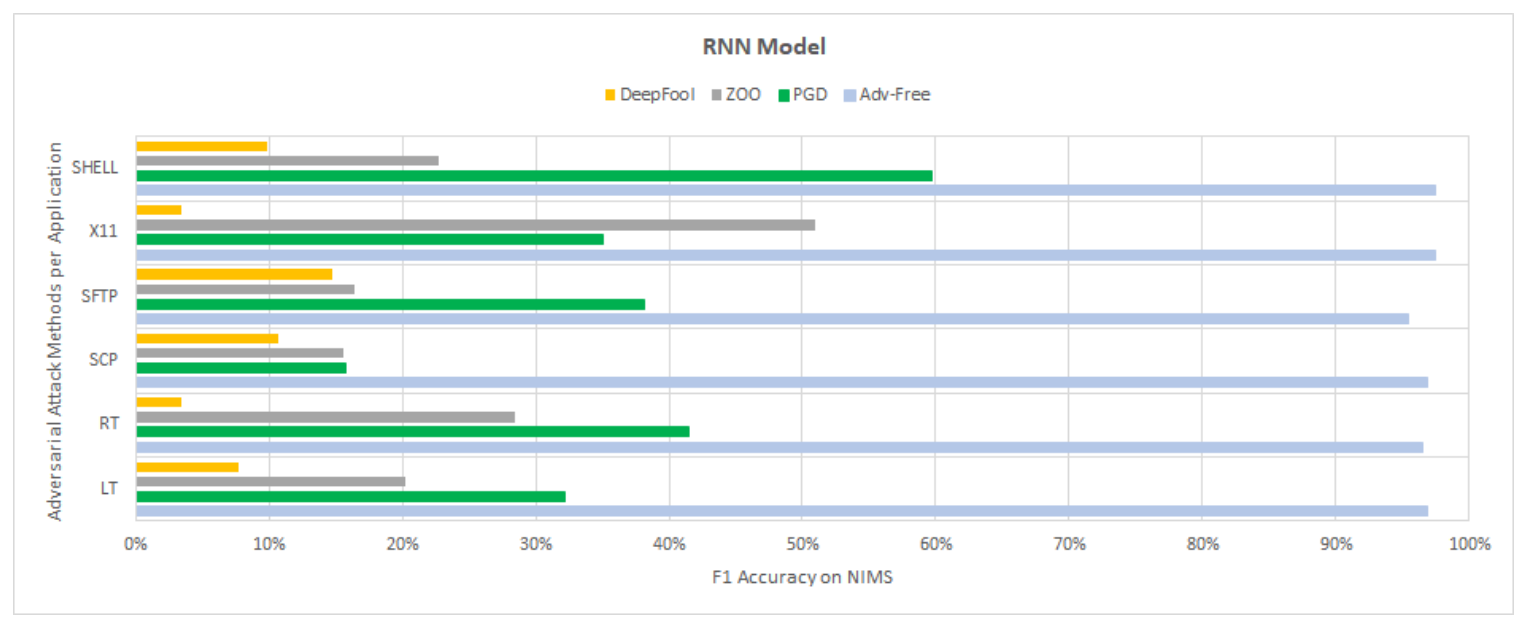

Figure 6.10: Effect of Adversarial Samples generated on RNN models trained by Adversarial-free NIMS dataset

less resilient to this type of attack compared to machine learning. We can also conclude that the DeepFool attack method has the highest effect on NIMS dataset, similar to the ISCX VPN-NonVPN dataset. 


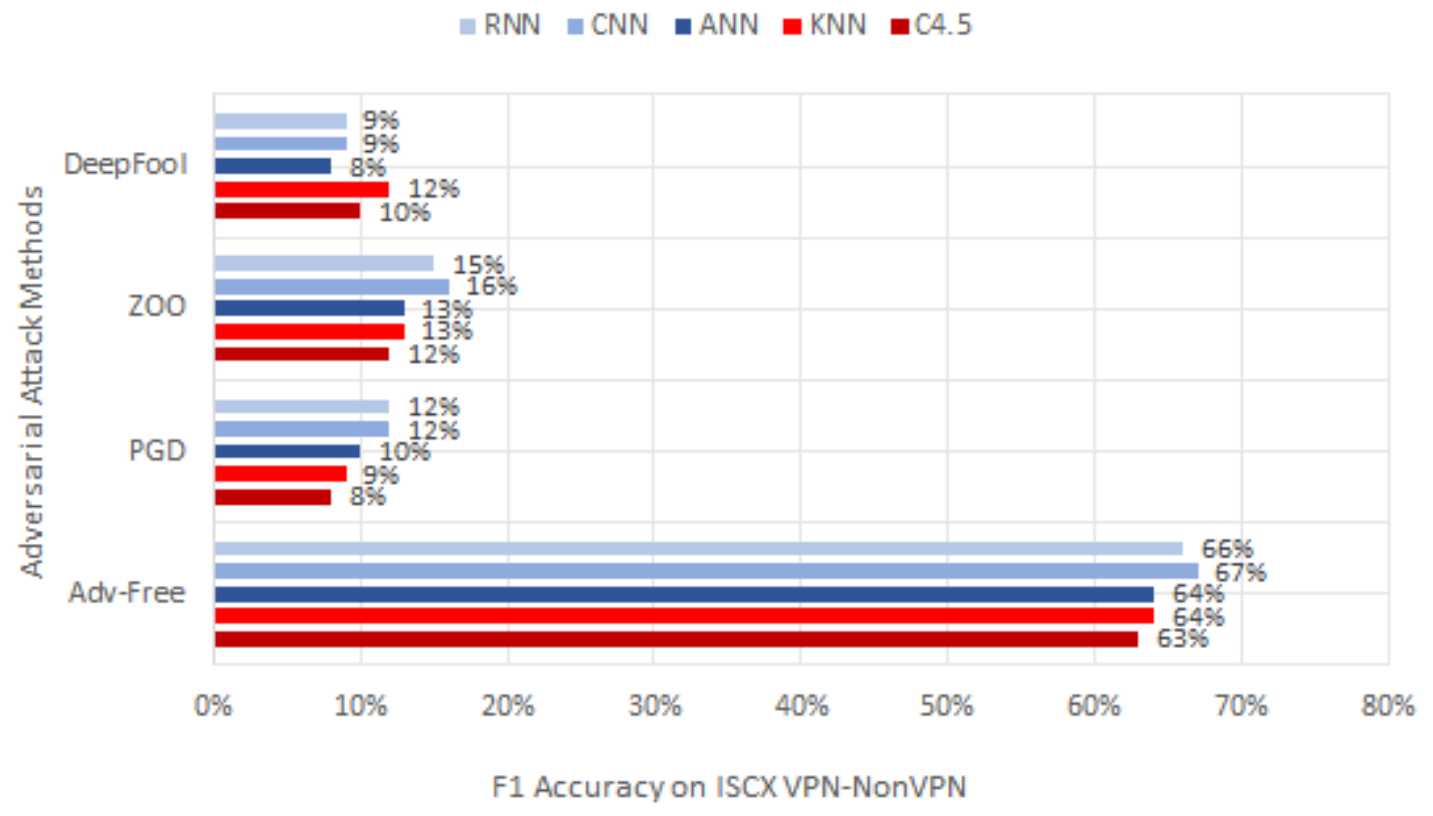

Figure 6.11: Effect of Adversarial Samples generated on five models trained by Adversarial-free ISCX VPN-NonVPN dataset. DL algorithms are shown in different shades of Blue. ML algorithms are shown in different shades of Red.

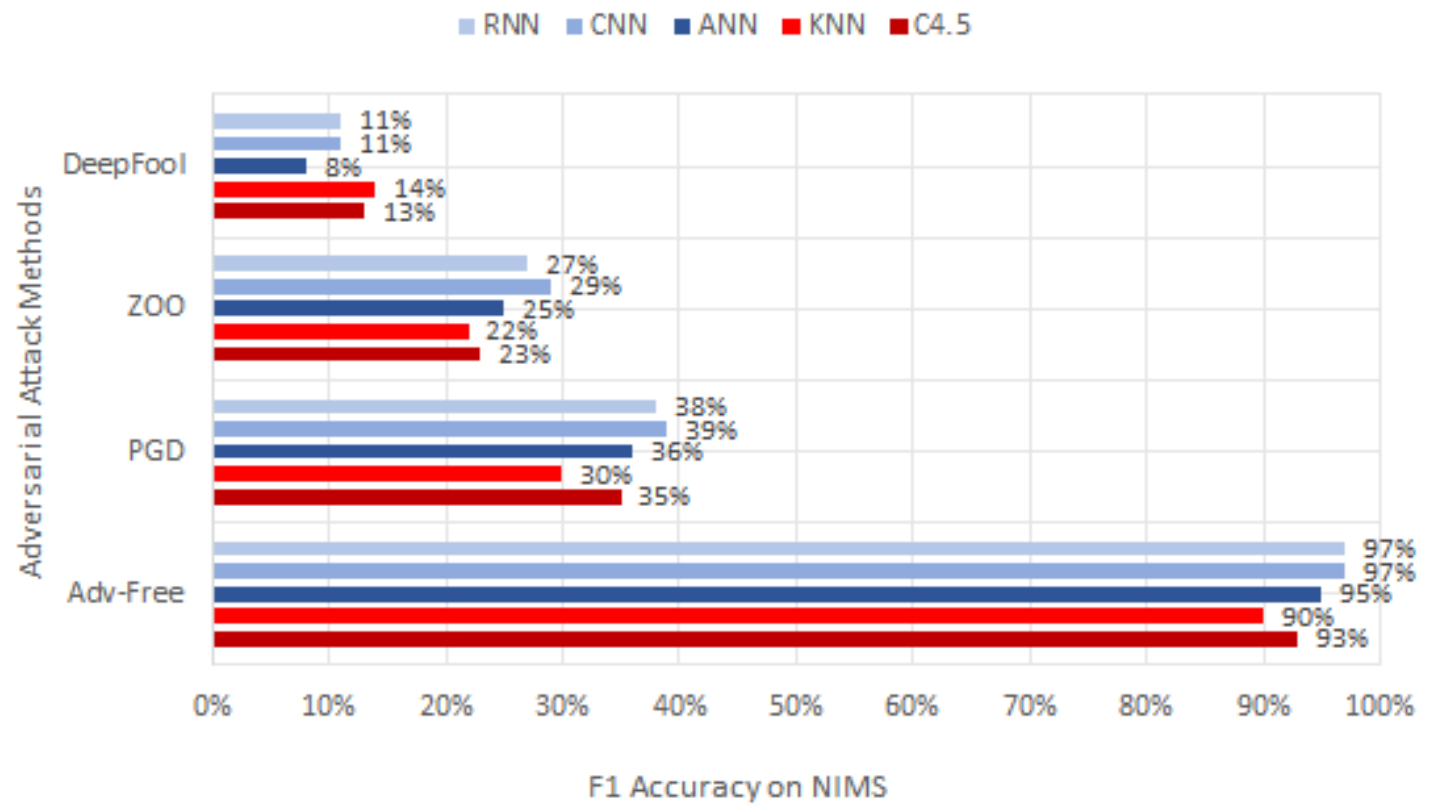

Figure 6.12: Effect of Adversarial Samples generated on five models trained by Adversarial-free NIMS dataset. DL algorithms are shown in different shades of Blue. ML algorithms are shown in different shades of Red. 


\section{Chapter 7}

\section{ML and DL Resilience against Adversarial Evasion Attacks: Experiments with Top Feature Selection}

This chapter reports and evaluates the learning model's resilience against the three adversarial evasion attacks: PGD, ZOO, and DeepFool, using the top nine features generated from the Mutual Information algorithm, as listed in Table 7.1. Our objective is to find if we can improve the model's performance in an adversarial-free environment and their resilience against adversarial attacks when using more top selected features. We will compare our results with the experimental results generated using the same top five features in Chapters 5 and 6 . First, we retrain the five models: C4.5, KNN, ANN, CNN, and RNN using Mutual Information for ISCX VPNNonVPN and NIMS in an adversarial-free environment with the same experimental setup in Chapter 5. Then, we evaluate the adversarially trained models' resilience in classifying encrypted traffic against adversarial samples generated from the same adversarial methods used in Chapter 6. 
Table 7.1: The Top 9 features selected from ISCX VPN-NonVPN and NIMS Datasets

\begin{tabular}{||c|l|c|l||}
\hline ID & \multicolumn{1}{|c|}{ ISCX } & ID & \multicolumn{1}{|c||}{ NIMS } \\
\hline \hline 4 & min-fiat & 8 & min-fiat \\
5 & max-fiat & 10 & max-fiat \\
6 & max-biat & 14 & max-biat \\
0 & duration & 1 & mean-fpktl \\
9 & flowPktsPerSecond & 3 & std-fpktl \\
10 & flowBytesPerSecond & 5 & mean-bpktl \\
12 & max-flowiat & 7 & std-bpktl \\
13 & mean-flowiat & 9 & mean-fiat \\
14 & std-flowiat & 15 & std-biat \\
\hline
\end{tabular}

\subsection{Evaluating ML Algorithms against Adversar- ial Attacks: Top Feature Selection}

\subsubsection{Performance on C4.5}

We will evaluate the C4.5 model's resilience in adversarial-free and adversarial attack environments on the same datasets: ISCX VPN-NonVPN and NIMS, using the same evasion attacks: PGD, ZOO, and DeepFool. Table 7.2 and 7.3 indicate the Precision and Recall of the model's resilience in classifying each encrypted application against the three adversarial attacks.

We noticed that PGD, ZOO, and DeepFool consider having the same effect on C4.5. In ISCX VPN-NonVPN, the F1-Score accuracy is reduced from $64 \%$ and $69 \%$ to $0 \%$ for Chat and VPN-FT using PGD adversarial samples, respectively. Similarly, as illustrated in Figure 7.1 with DeepFool adversarial samples, the accuracy of Mail 
and VoIP degraded to $0 \%$, respectively.

In Figure 7.2, we can see an accuracy degradation in NIMS from $99 \%$ and $99 \%$ to $17 \%$ and $6 \%$ for RT and Shell using DeepFool adversarial samples, respectively. We also can see that the F1-Score decreased to $95 \%$ and $49 \%$ using ZOO adversarial samples, respectively.

We can conclude that the performance of $\mathrm{C} 4.5$ in the adversarial-free environment in NIMS was more resilient to the three adversarial attacks in classifying encrypted traffic compared to ISCX VPN-NonVPN. We also find that the DeepFool attack has the most influence on C4.5 models on NIMS dataset. Unlike the ISCX VPN-NonVPN dataset, where C4.5 models are less resilient to PGD evasion attacks. The effect of the ZOO attack is on average on both datasets compared to the other two evasion attacks. 
Table 7.2: Evaluation Metric Results (\%) of C4.5 models in attack environment using Top FS for ISCX VPN-NonVPN

\begin{tabular}{||l|c|c|c|c|c|c||}
\hline Class & \multicolumn{5}{c|}{ Attack Methods } \\
\hline \hline- & \multicolumn{2}{|c|}{ PGD } & \multicolumn{2}{c|}{ ZOO } & \multicolumn{2}{c||}{ DeepFool } \\
\hline - & Precision & Recall & Precision & Recall & Precision & Recall \\
\hline Browsing & 7 & 11 & 21 & 15 & 36 & 29 \\
\hline Chat & 0 & 0 & 11 & 7 & 26 & 5 \\
\hline Streaming & 3 & 20 & 4 & 9 & 7 & 1 \\
\hline Mail & 0 & 0 & 6 & 8 & 0 & 0 \\
\hline VoIP & 1 & 0 & 4 & 1 & 0 & 0 \\
\hline P2P & 5 & 10 & 9 & 6 & 28 & 4 \\
\hline File Transfer & 4 & 8 & 3 & 6 & 1 & 7 \\
\hline VPN-Browsing & 3 & 0 & 1 & 0 & 1 & 1 \\
\hline VPN-Chat & 20 & 5 & 9 & 16 & 24 & 5 \\
\hline VPN-Streaming & 2 & 3 & 1 & 1 & 12 & 43 \\
\hline VPN-Mail & 11 & 10 & 9 & 10 & 17 & 12 \\
\hline VPN-VoIP & 20 & 2 & 14 & 13 & 12 & 3 \\
\hline VPN-P2P & 13 & 45 & 6 & 9 & 20 & 7 \\
\hline VPN-File Transfer & 0 & 0 & 9 & 8 & 10 & 11 \\
\hline Average & 6 & 8 & 8 & 8 & 14 & 9 \\
\hline
\end{tabular}

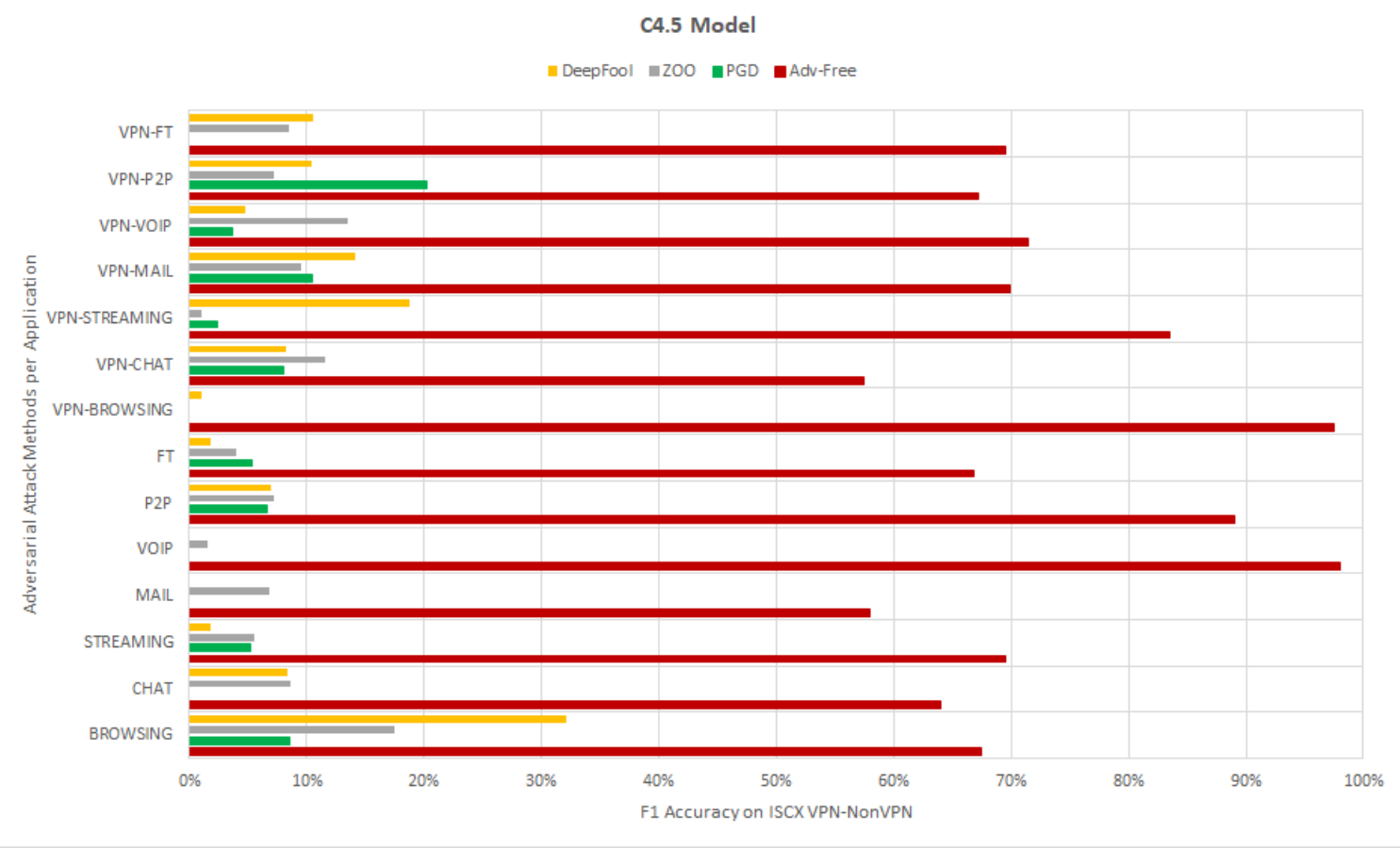


Table 7.3: Evaluation Metric Results (\%) of C4.5 models in attack environment using Top FS for NIMS dataset

\begin{tabular}{||l|c|c|c|c|c|c||}
\hline Class & \multicolumn{5}{c||}{ Attack Methods } \\
\hline \hline- & \multicolumn{2}{|c|}{ PGD } & \multicolumn{2}{c|}{ ZOO } & \multicolumn{2}{c||}{ DeepFool } \\
\hline- & Precision & Recall & Precision & Recall & Precision & Recall \\
\hline Local Tunneling & 0 & 0 & 1 & 0 & 17 & 59 \\
\hline Remote Tunneling & 92 & 99 & 91 & 100 & 11 & 38 \\
\hline SCP & 5 & 9 & 1 & 2 & 10 & 1 \\
\hline SFTP & 0 & 0 & 0 & 0 & 15 & 16 \\
\hline X11 & 91 & 97 & 1 & 1 & 31 & 50 \\
\hline Shell & 90 & 63 & 45 & 53 & 7 & 5 \\
\hline Average & 46 & 45 & 23 & 26 & 15 & 28 \\
\hline
\end{tabular}

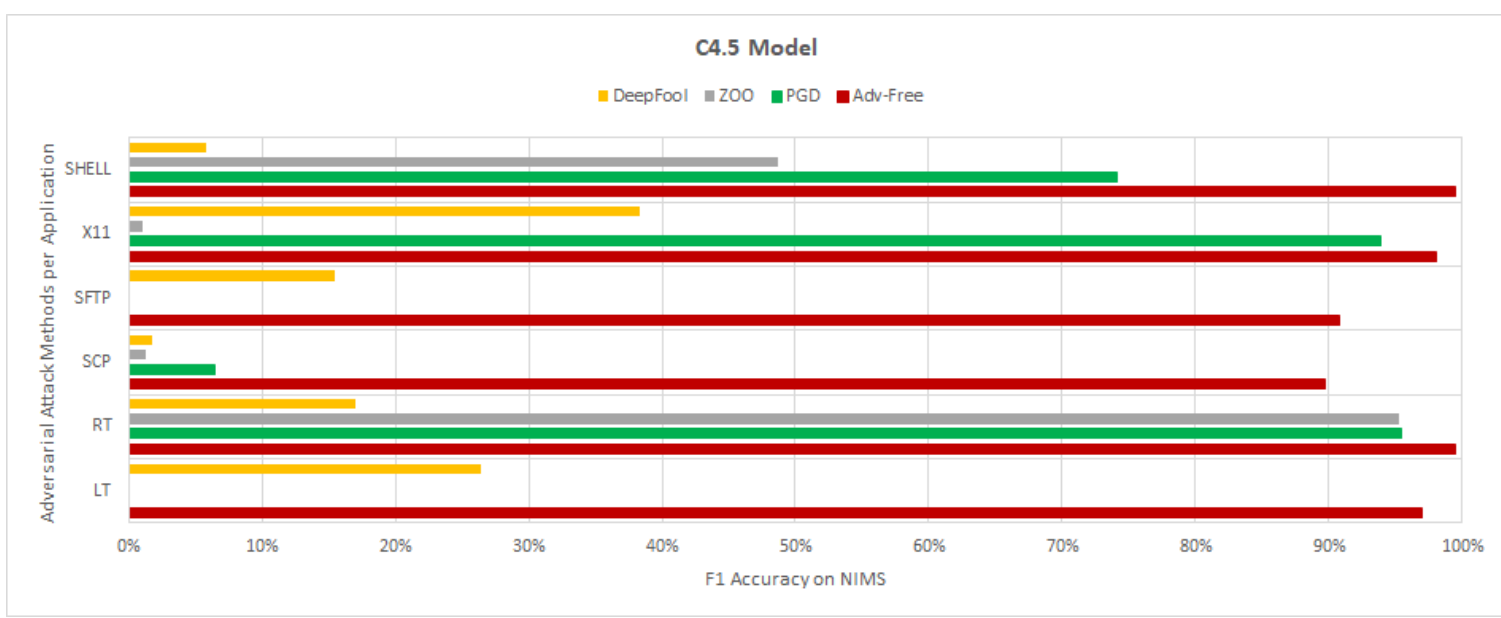

Figure 7.2: Effect of Adv. Samples generated on C4.5 models using Top FS trained by Adversarial-free NIMS dataset

\subsubsection{Performance on KNN}

For ISCX VPN-NonVPN and NIMS datasets, we illustrate the KNN model's resilience in classifying encrypted traffic against the PGD, ZOO, and DeepFool adversarial 
evasion attacks by presenting each application's Precision and Recall, as shown in Table 7.4 and 7.5. Figure 7.3 and 7.4 show the F1-Score calculations used to evaluate the model's performance and compare the results to that from the adversarial-free environment.

We observe in ISCX VPN-NonVPN, as shown in Figure 7.3, an F1-Score accuracy reduction from $82 \%, 40 \%, 81 \%$, and $51 \%$ to $0 \%$ for P2P, VPN-Chat, VPN-Streaming, and VPN-P2P using PGD adversarial attack, respectively. Similarly, as illustrated in Figure 7.3 with ZOO and Deepfool adversarial samples, the accuracy of KNN degraded to $8 \%, 22 \%, 16 \%, 32 \%$ and $17 \%, 11 \%, 10 \%, 12 \%$ respectively.

The F1-Score accuracy is tremendously reduced from $94 \%$ and $99 \%$ to $16 \%$ and $15 \%$ for RT, and Shell using DeepFool adversarial attack in NIMS, respectively. We can notice a slight drop in the F1-Score accuracy when using PGD and ZOO attacks to $88 \%, 84 \%$ and $92 \%, 71 \%$, respectively, as illustrated in Figure 7.4 .

We can conclude that the resilience of KNN in classifying encrypted traffic was less against PGD attacks in ISCX VPN-NonVPN compared to ZOO and DeepFool attacks where KNN models were more resilient to them. While in NIMS, KNN was more resilient to PGD attacks compared to DeepFool that has more influence on KNN models. 
Table 7.4: Evaluation Metric Results (\%) of KNN models in attack environment using Top FS for ISCX VPN-NonVPN

\begin{tabular}{||l|c|c|c|c|c|c||}
\hline Class & \multicolumn{5}{c||}{ Attack Methods } \\
\hline \hline- & \multicolumn{2}{|c|}{ PGD } & \multicolumn{2}{c||}{ ZOO } & \multicolumn{2}{c||}{ DeepFool } \\
\hline- & Precision & Recall & Precision & Recall & Precision & Recall \\
\hline Browsing & 4 & 12 & 23 & 36 & 29 & 31 \\
\hline Chat & 21 & 3 & 50 & 2 & 11 & 8 \\
\hline Streaming & 100 & 1 & 4 & 12 & 5 & 10 \\
\hline Mail & 0 & 0 & 0 & 0 & 10 & 28 \\
\hline VoIP & 0 & 0 & 0 & 0 & 5 & 1 \\
\hline P2P & 0 & 0 & 10 & 7 & 25 & 13 \\
\hline File Transfer & 15 & 7 & 26 & 26 & 3 & 14 \\
\hline VPN-Browsing & 13 & 37 & 11 & 1 & 1 & 0 \\
\hline VPN-Chat & 0 & 0 & 18 & 29 & 8 & 17 \\
\hline VPN-Streaming & 0 & 0 & 10 & 37 & 15 & 7 \\
\hline VPN-Mail & 10 & 3 & 13 & 22 & 15 & 27 \\
\hline VPN-VoIP & 30 & 6 & 23 & 14 & 23 & 8 \\
\hline VPN-P2P & 0 & 0 & 23 & 53 & 12 & 13 \\
\hline VPN-File Transfer & 22 & 5 & 43 & 3 & 14 & 8 \\
\hline Average & 15 & 5 & 18 & 17 & 13 & 13 \\
\hline
\end{tabular}

\subsection{Evaluating DL Algorithms against Adversarial}

\section{Attacks: Top Feature Selection}

\subsubsection{Performance on ANN}

As shown in Table 7.6 and 7.7, we calculate the Precision and Recall of each encrypted application in ISCX VPN-NonVPN and NIMS datasets against the three adversarial 
Table 7.5: Evaluation Metric Results (\%) of KNN models in attack environment using Top FS for NIMS dataset

\begin{tabular}{||l|c|c|c|c|c|c||}
\hline \multirow{2}{*}{ Class } & \multicolumn{5}{c||}{ Attack Methods } \\
\hline \hline- & \multicolumn{2}{|c|}{ PGD } & \multicolumn{2}{c|}{ ZOO } & \multicolumn{2}{c||}{ DeepFool } \\
\hline - & Precision & Recall & Precision & Recall & Precision & Recall \\
\hline Local Tunneling & 0 & 0 & 1 & 2 & 19 & 64 \\
\hline Remote Tunneling & 80 & 98 & 97 & 99 & 14 & 18 \\
\hline SCP & 12 & 10 & 2 & 9 & 3 & 5 \\
\hline SFTP & 20 & 19 & 0 & 0 & 95 & 17 \\
\hline X11 & 95 & 94 & 0 & 4 & 28 & 36 \\
\hline Shell & 93 & 77 & 63 & 81 & 17 & 14 \\
\hline Average & 50 & 50 & 27 & 33 & 29 & 26 \\
\hline
\end{tabular}

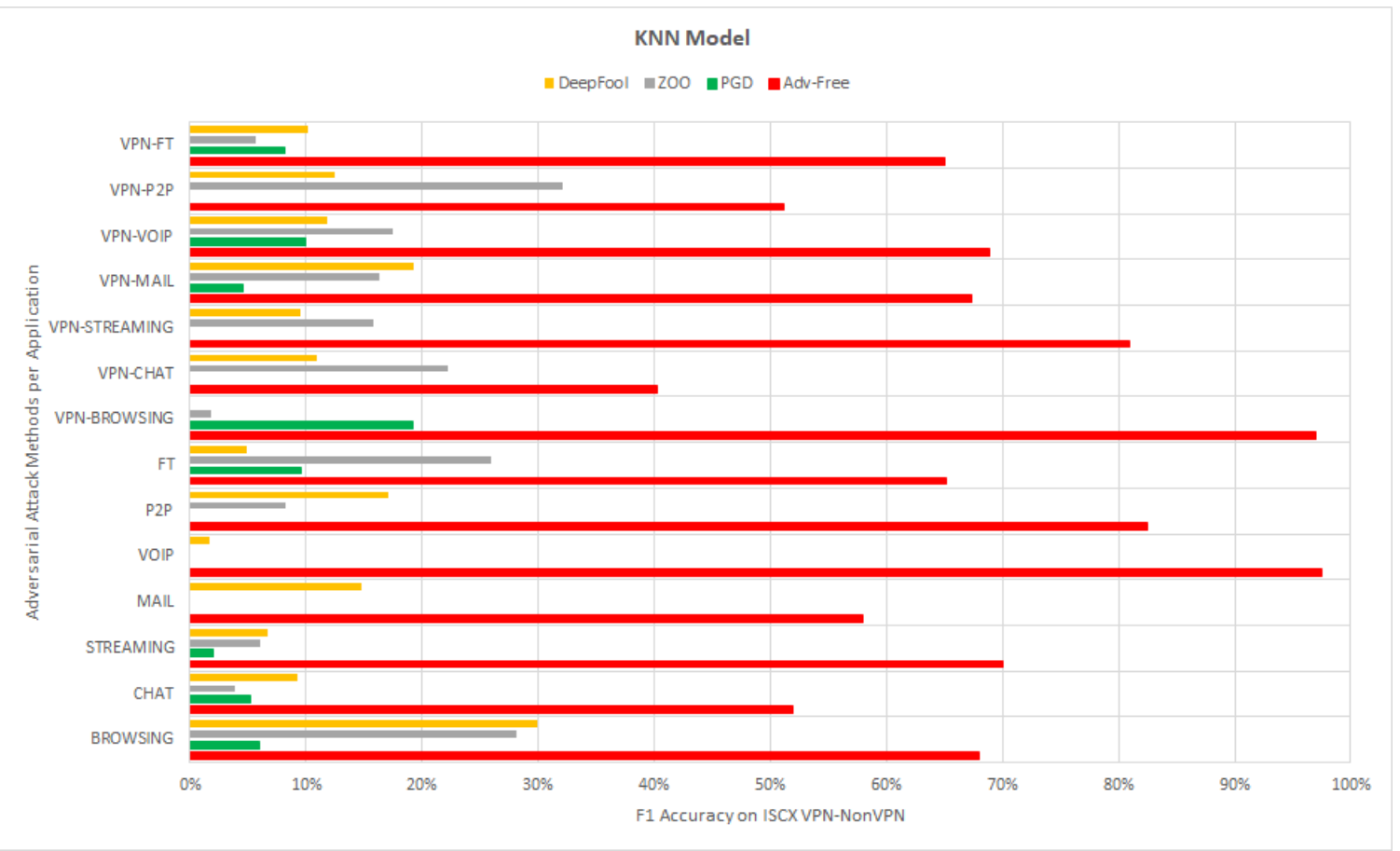

Figure 7.3: Effect of Adv. Samples generated on KNN models using Top FS trained by Adversarial-free ISCX VPN-NonVPN dataset 


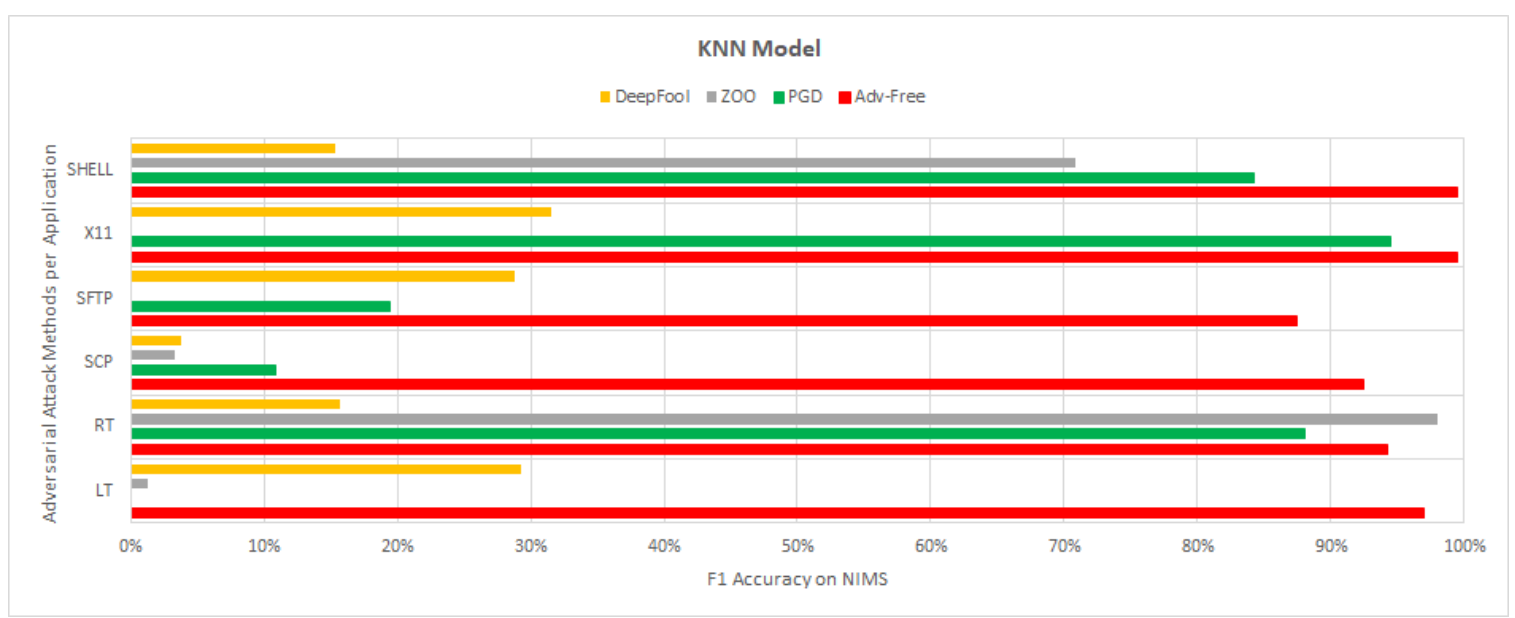

Figure 7.4: Effect of Adv. Samples generated on KNN models using Top FS trained by Adversarial-free NIMS dataset

attacks. We also used these results to determine the F1-Score accuracy to evaluate the model's performance and resilience, as shown in Figure 7.5 and 7.6.

If we take a closer look at ANN in ISCX VPN-NonVPN, we find that PGD, ZOO, and DeepFool consider having the same effect and the overall accuracy is reduced by almost 85\%, as shown in Figure 7.5. PGD attack has the most considerable influence on the ANN on ISCX VPN-NonVPN.

We can see from Figure 7.6 that the F1-Score accuracy is reduced from 97\%, 91\%, and $98 \%$ to $0 \%, 3 \%$, and $2 \%$ for RT, SFTP, and X11 using DeepFool adversarial attack, respectively. We can notice that the PGD and ZOO attacks decreased the F1-Score accuracy to 49\%,22\%,35\% and 23\%,20\%, 52\%, respectively, as illustrated in Figure 7.6.

We can conclude that the performance of ANN in the adversarial-free environment in NIMS was more resilient to the three adversarial attacks in classifying encrypted traffic compared to ISCX VPN-NonVPN. We also find that the DeepFool attack has 
Table 7.6: Evaluation Metric Results (\%) of ANN models in attack environment using Top FS for ISCX VPN-NonVPN

\begin{tabular}{||l|c|c|c|c|c|c||}
\hline Class & \multicolumn{5}{c||}{ Attack Methods } \\
\hline \hline- & \multicolumn{2}{|c|}{ PGD } & \multicolumn{2}{c||}{ ZOO } & \multicolumn{2}{c||}{ DeepFool } \\
\hline- & Precision & Recall & Precision & Recall & Precision & Recall \\
\hline Browsing & 19 & 10 & 10 & 18 & 9 & 16 \\
\hline Chat & 4 & 4 & 41 & 1 & 17 & 10 \\
\hline Streaming & 31 & 39 & 9 & 1 & 2 & 8 \\
\hline Mail & 0 & 0 & 1 & 1 & 0 & 0 \\
\hline VoIP & 1 & 1 & 0 & 0 & 0 & 0 \\
\hline P2P & 2 & 3 & 0 & 0 & 7 & 1 \\
\hline File Transfer & 33 & 26 & 14 & 1 & 8 & 15 \\
\hline VPN-Browsing & 0 & 0 & 15 & 28 & 40 & 32 \\
\hline VPN-Chat & 27 & 8 & 23 & 20 & 29 & 18 \\
\hline VPN-Streaming & 44 & 11 & 37 & 19 & 6 & 18 \\
\hline VPN-Mail & 7 & 12 & 29 & 20 & 18 & 12 \\
\hline VPN-VoIP & 3 & 0 & 25 & 15 & 18 & 20 \\
\hline VPN-P2P & 0 & 0 & 27 & 39 & 20 & 17 \\
\hline VPN-File Transfer & 10 & 9 & 16 & 40 & 10 & 11 \\
\hline Average & 13 & 9 & 18 & 15 & 13 & 13 \\
\hline
\end{tabular}

the most influence on ANN models on both datasets.

\subsubsection{Performance on CNN}

In this experiment, we show the CNN model's resilience per each encrypted application against the three adversarial attack's Precision and Recall, as shown in Table 7.8 and 7.9. We calculate the F1-Score and present the results in Figure 7.7 and 7.8, to evaluate the model's performance and compare the results of the adversarial samples 
Table 7.7: Evaluation Metric Results (\%) of ANN models in attack environment using Top FS for NIMS dataset

\begin{tabular}{||l|c|c|c|c|c|c||}
\hline Class & \multicolumn{5}{c||}{ Attack Methods } \\
\hline \hline- & \multicolumn{2}{|c|}{ PGD } & \multicolumn{2}{c|}{ ZOO } & \multicolumn{2}{c||}{ DeepFool } \\
\hline- & Precision & Recall & Precision & Recall & Precision & Recall \\
\hline Local Tunneling & 40 & 36 & 0 & 0 & 0 & 0 \\
\hline Remote Tunneling & 82 & 99 & 91 & 98 & 87 & 99 \\
\hline SCP & 29 & 41 & 4 & 5 & 2 & 4 \\
\hline SFTP & 12 & 2 & 0 & 0 & 0 & 0 \\
\hline X11 & 63 & 99 & 95 & 90 & 48 & 51 \\
\hline Shell & 95 & 69 & 86 & 72 & 13 & 2 \\
\hline Average & 54 & 58 & 46 & 44 & 25 & 26 \\
\hline
\end{tabular}

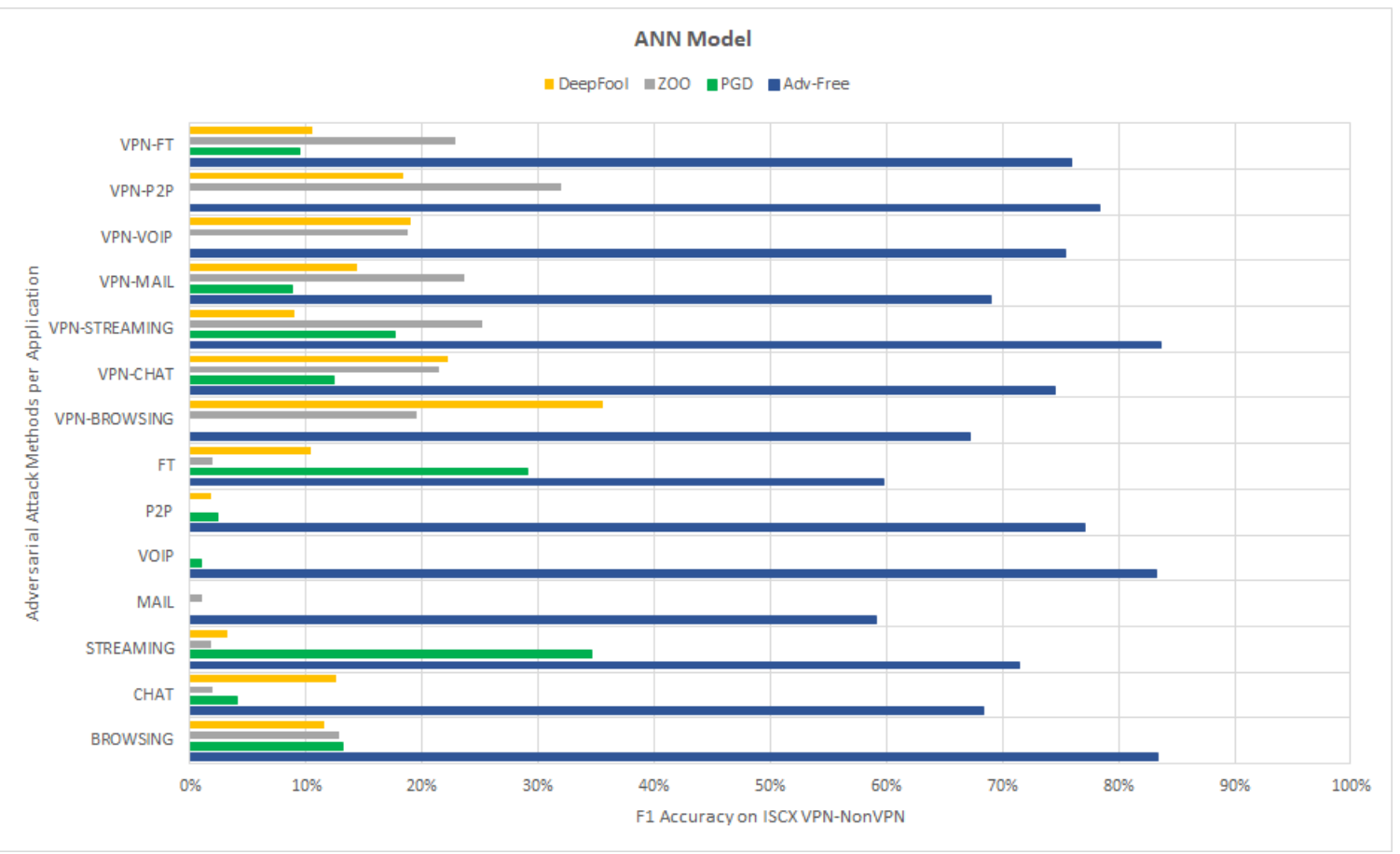

Figure 7.5: Effect of Adv. Samples generated on ANN models using Top FS trained by Adversarial-free ISCX VPN-NonVPN dataset 


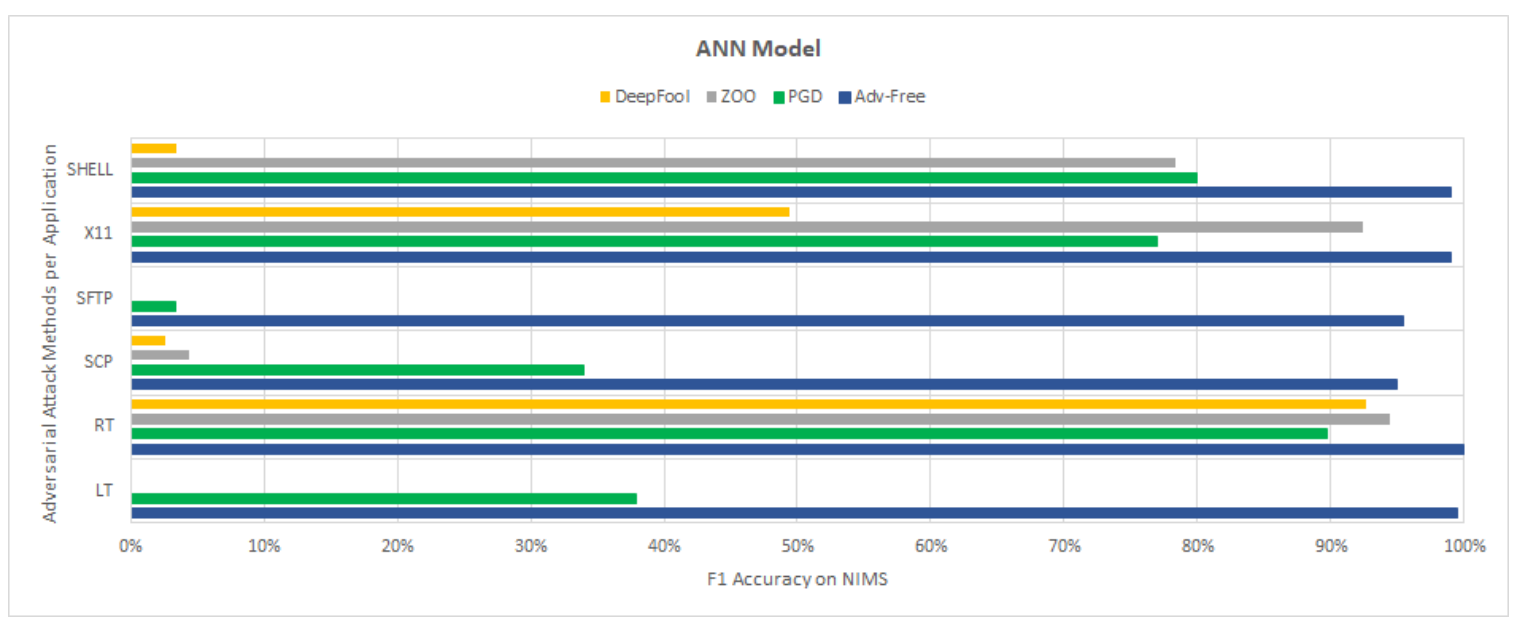

Figure 7.6: Effect of Adv. Samples generated on ANN models using Top FS trained by Adversarial-free NIMS dataset

to that from the adversarial-free environment.

In general, we noticed that the F1-Score accuracy of CNN in ISCX VPN-NonVPN is reduced from $81 \%, 86 \%, 83 \%$, and $85 \%$ to $9 \%, 0 \%, 8 \%$, and $6 \%$ for Chat, VPNBrowsing, VPN-Streaming, and VPN-VoIP using PGD adversarial attack, respectively, as illustrated in Figure 7.7. We also noticed that with ZOO and Deepfool adversarial samples, the accuracy of RNN degraded to $33 \%, 14 \%, 20 \%, 20 \%$ and $13 \%, 10 \%, 14 \%, 22 \%$, respectively.

We find that the accuracy in NIMS was roughly reduced from $100 \%$, and $99 \%$ to $56 \%$, and 3\% for RT, and Shell, using DeepFool adversarial samples, respectively. At the same time, in Figure 7.8, accuracy decreased to 93\%, 98\%, and 99\%, 53\% using PGD and ZOO adversarial samples, respectively.

We can conclude that the resilience of $\mathrm{CNN}$ in classifying encrypted traffic was less against PGD attacks in ISCX VPN-NonVPN compared to ZOO and DeepFool attacks where CNN models were more resilient to them. While in NIMS, CNN was more resilient to PGD attacks compared to DeepFool that has more impact on RNN 
Table 7.8: Evaluation Metric Results (\%) of CNN models in attack environment using Top FS for ISCX VPN-NonVPN

\begin{tabular}{||l|c|c|c|c|c|c||}
\hline Class & \multicolumn{5}{c||}{ Attack Methods } \\
\hline \hline- & \multicolumn{2}{|c|}{ PGD } & \multicolumn{2}{c|}{ ZOO } & \multicolumn{2}{c||}{ DeepFool } \\
\hline- & Precision & Recall & Precision & Recall & Precision & Recall \\
\hline Browsing & 19 & 27 & 34 & 19 & 27 & 11 \\
\hline Chat & 6 & 19 & 43 & 27 & 16 & 11 \\
\hline Streaming & 18 & 7 & 40 & 11 & 21 & 36 \\
\hline Mail & 20 & 1 & 17 & 9 & 0 & 0 \\
\hline VoIP & 0 & 0 & 21 & 3 & 0 & 0 \\
\hline P2P & 17 & 21 & 30 & 27 & 20 & 10 \\
\hline File Transfer & 30 & 27 & 22 & 41 & 24 & 16 \\
\hline VPN-Browsing & 0 & 0 & 21 & 11 & 11 & 10 \\
\hline VPN-Chat & 1 & 8 & 19 & 19 & 20 & 1 \\
\hline VPN-Streaming & 17 & 5 & 21 & 20 & 18 & 12 \\
\hline VPN-Mail & 19 & 23 & 48 & 50 & 5 & 4 \\
\hline VPN-VoIP & 4 & 9 & 21 & 19 & 29 & 18 \\
\hline VPN-P2P & 24 & 12 & 13 & 22 & 24 & 38 \\
\hline VPN-File Transfer & 9 & 10 & 14 & 24 & 10 & 21 \\
\hline Average & 13 & 12 & 26 & 22 & 16 & 13 \\
\hline
\end{tabular}

models.

\subsubsection{Performance on RNN}

Consistently with the Precision and Recall results of the ISCX VPN-NonVPN and NIMS datasets, as shown in Table 7.10 and 7.11, we can calculate the F1-Score to evaluate the model's performance and compare the results of the adversarial samples 
Table 7.9: Evaluation Metric Results (\%) of CNN models in attack environment using Top FS for NIMS dataset

\begin{tabular}{||l|c|c|c|c|c|c||}
\hline Class & \multicolumn{5}{c||}{ Attack Methods } \\
\hline \hline- & \multicolumn{2}{|c|}{ PGD } & \multicolumn{2}{c|}{ ZOO } & \multicolumn{2}{c||}{ DeepFool } \\
\hline- & Precision & Recall & Precision & Recall & Precision & Recall \\
\hline Local Tunneling & 19 & 25 & 0 & 1 & 69 & 58 \\
\hline Remote Tunneling & 88 & 99 & 99 & 99 & 39 & 99 \\
\hline SCP & 27 & 20 & 4 & 8 & 8 & 2 \\
\hline SFTP & 50 & 53 & 0 & 0 & 0 & 0 \\
\hline X11 & 100 & 98 & 90 & 98 & 38 & 56 \\
\hline Shell & 99 & 97 & 49 & 58 & 4 & 3 \\
\hline Average & 64 & 65 & 40 & 44 & 26 & 36 \\
\hline
\end{tabular}

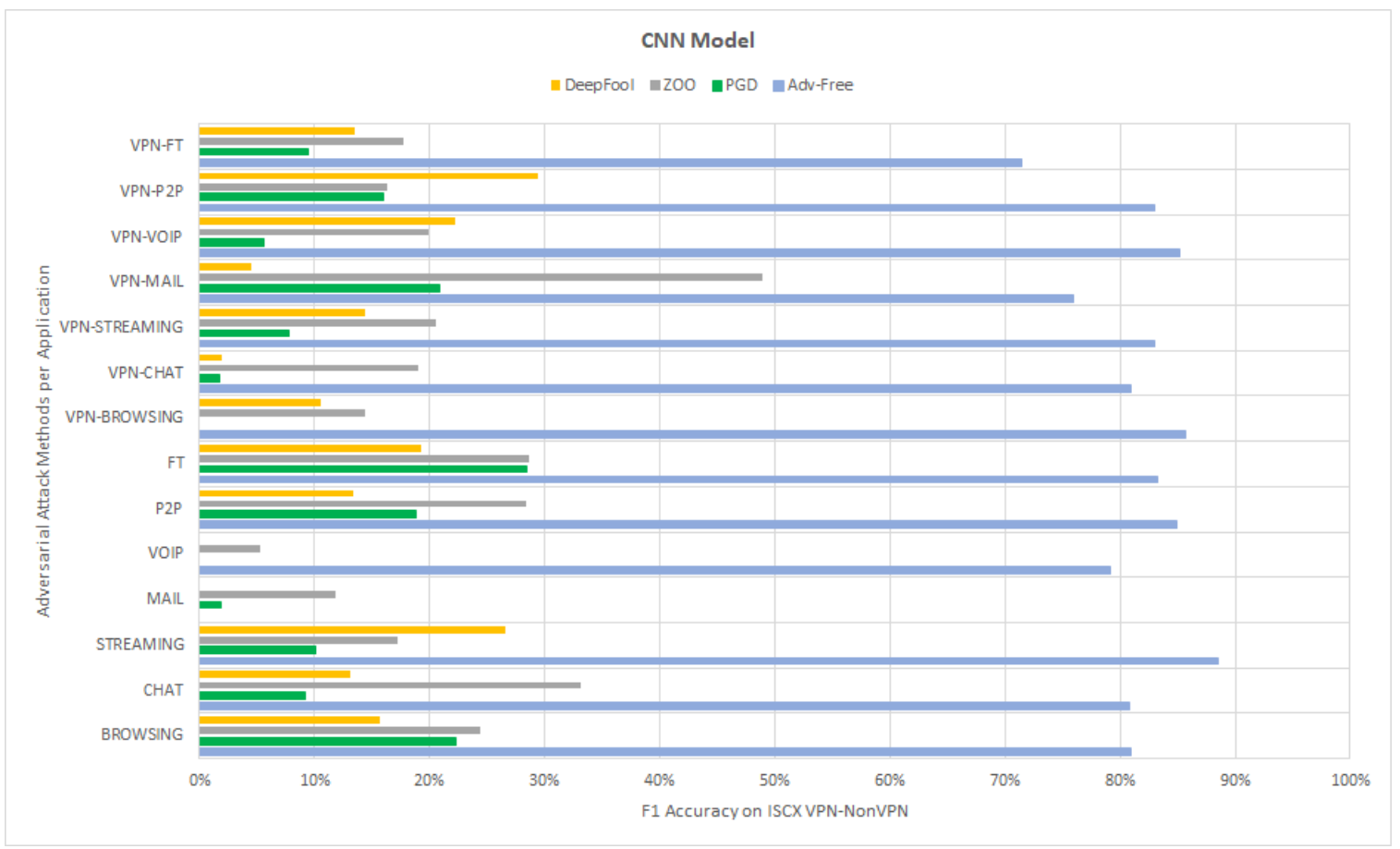

Figure 7.7: Effect of Adv. Samples generated on CNN models using Top FS trained by Adversarial-free ISCX VPN-NonVPN dataset 


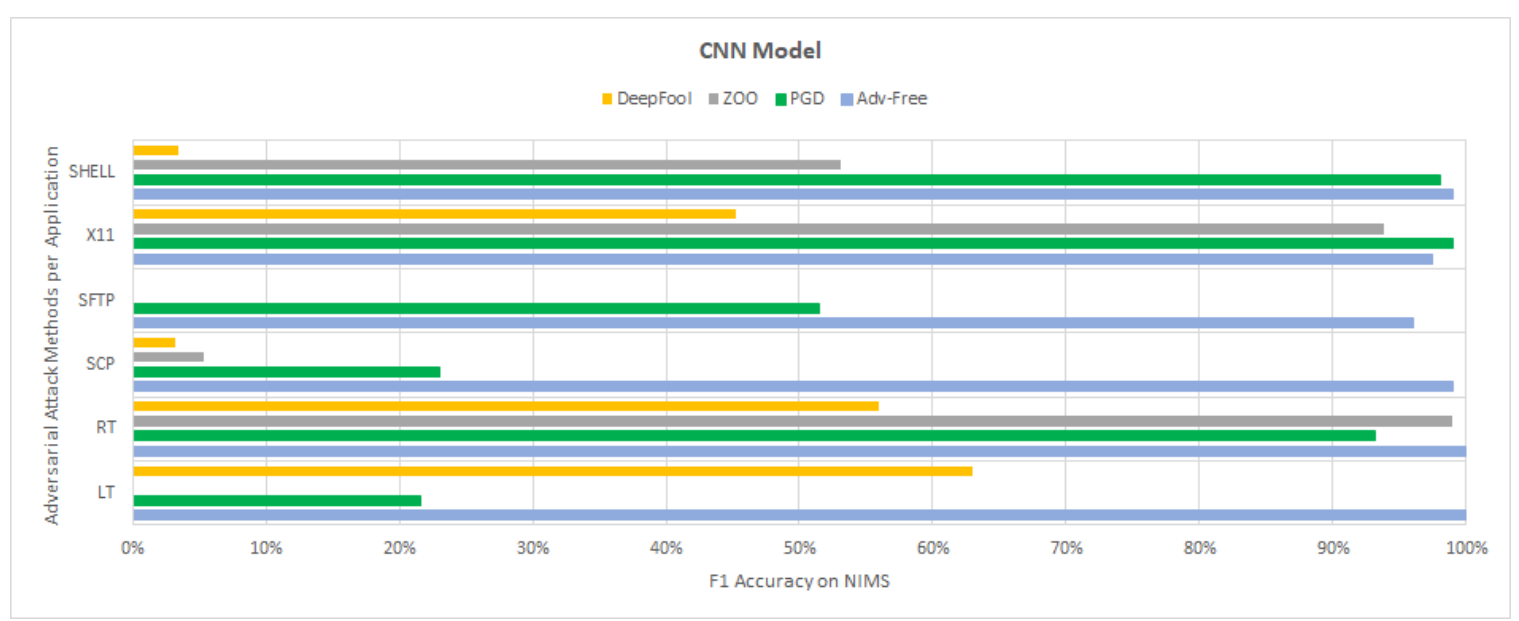

Figure 7.8: Effect of Adv. Samples generated on CNN models using Top FS trained by Adversarial-free NIMS dataset

to that from the adversarial-free environment, as shown in Figure 7.9 and 7.10.

The RNN performance in ISCX VPN-NonVPN when using the F1-Score results shows a degradation from $89 \%, 77 \%$, and $87 \%$ to $0 \%, 7 \%$, and $7 \%$ for VPN-Browsing, VPN-Streaming, and VPN-VoIP using PGD adversarial attack, respectively. Same with ZOO and Deepfool adversarial samples, the accuracy of RNN degraded to $12 \%$, $29 \%, 18 \%$ and $25 \%, 17 \%, 20 \%$, respectively, as illustrated in Figure 7.9.

While we observe in Figure 7.10 that the F1-Score accuracy in NIMS is significantly reduced from $100 \%, 99 \%$, and $99 \%$ to $50 \%, 57 \%$, and $18 \%$ for RT, X11, and Shell using DeepFool adversarial samples, respectively. Also, the accuracy decreased to $98 \%, 97 \%, 96 \%$ and $98 \%, 86 \%, 68 \%$ using PGD and ZOO adversarial samples, respectively.

We can conclude that the resilience of RNN in classifying encrypted traffic was less against PGD attack in ISCX VPN-NonVPN compared to ZOO attack where RNN models were more resilient to it. While in NIMS, RNN was more resilient to PGD 
Table 7.10: Evaluation Metric Results (\%) of RNN models in attack environment using Top FS for ISCX VPN-NonVPN

\begin{tabular}{||l|c|c|c|c|c|c||}
\hline Class & \multicolumn{5}{c||}{ Attack Methods } \\
\hline \hline- & \multicolumn{2}{|c|}{ PGD } & \multicolumn{2}{c|}{ ZOO } & \multicolumn{2}{c||}{ DeepFool } \\
\hline- & Precision & Recall & Precision & Recall & Precision & Recall \\
\hline Browsing & 21 & 14 & 20 & 22 & 14 & 7 \\
\hline Chat & 8 & 7 & 23 & 46 & 8 & 11 \\
\hline Streaming & 7 & 15 & 30 & 39 & 18 & 29 \\
\hline Mail & 23 & 10 & 14 & 12 & 0 & 0 \\
\hline VoIP & 0 & 0 & 14 & 46 & 0 & 0 \\
\hline P2P & 9 & 9 & 37 & 21 & 3 & 1 \\
\hline File Transfer & 22 & 32 & 12 & 35 & 9 & 19 \\
\hline VPN-Browsing & 0 & 0 & 15 & 10 & 30 & 21 \\
\hline VPN-Chat & 11 & 10 & 19 & 31 & 14 & 3 \\
\hline VPN-Streaming & 8 & 6 & 26 & 33 & 33 & 11 \\
\hline VPN-Mail & 7 & 27 & 33 & 52 & 30 & 24 \\
\hline VPN-VoIP & 14 & 5 & 24 & 15 & 18 & 22 \\
\hline VPN-P2P & 27 & 34 & 13 & 27 & 21 & 43 \\
\hline VPN-File Transfer & 16 & 9 & 22 & 39 & 20 & 35 \\
\hline Average & 12 & 13 & 22 & 31 & 16 & 16 \\
\hline
\end{tabular}

attacks compared to DeepFool that has more impact on RNN models.

\subsection{Performance Comparison of $\mathrm{ML}$ and $\mathrm{DL}$ in Adv. Environment using Top FS}

In this experiment, we build the learning models with different architecture, and we preprocess the benchmark datasets: ISCX VPN-NonVPN and NIMS using Mutual 
Table 7.11: Evaluation Metric Results (\%) of RNN models in attack environment using Top FS for NIMS dataset

\begin{tabular}{||l|c|c|c|c|c|c||}
\hline Class & \multicolumn{5}{|c||}{ Attack Methods } \\
\hline \hline- & \multicolumn{2}{|c|}{ PGD } & \multicolumn{2}{c||}{ ZOO } & \multicolumn{2}{c||}{ DeepFool } \\
\hline - & Precision & Recall & Precision & Recall & Precision & Recall \\
\hline Local Tunneling & 26 & 20 & 0 & 0 & 39 & 73 \\
\hline Remote Tunneling & 97 & 100 & 100 & 97 & 44 & 58 \\
\hline SCP & 19 & 28 & 2 & 3 & 13 & 15 \\
\hline SFTP & 59 & 45 & 0 & 0 & 0 & 0 \\
\hline X11 & 95 & 99 & 80 & 92 & 57 & 58 \\
\hline Shell & 100 & 92 & 74 & 63 & 30 & 13 \\
\hline Average & 66 & 64 & 43 & 43 & 31 & 36 \\
\hline
\end{tabular}

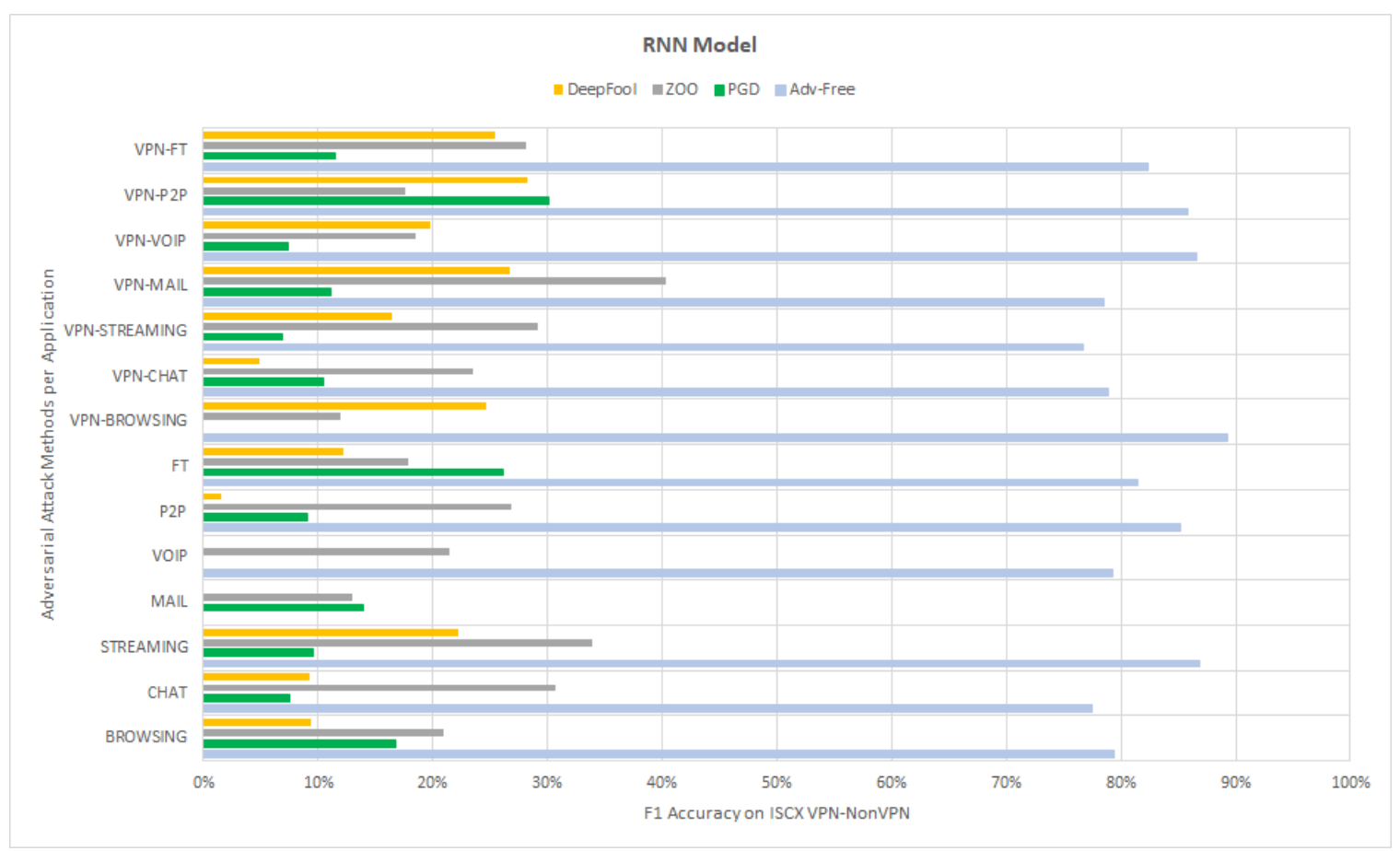

Figure 7.9: Effect of Adv. Samples generated on RNN models using Top FS trained by Adversarial-free ISCX VPN-NonVPN dataset 


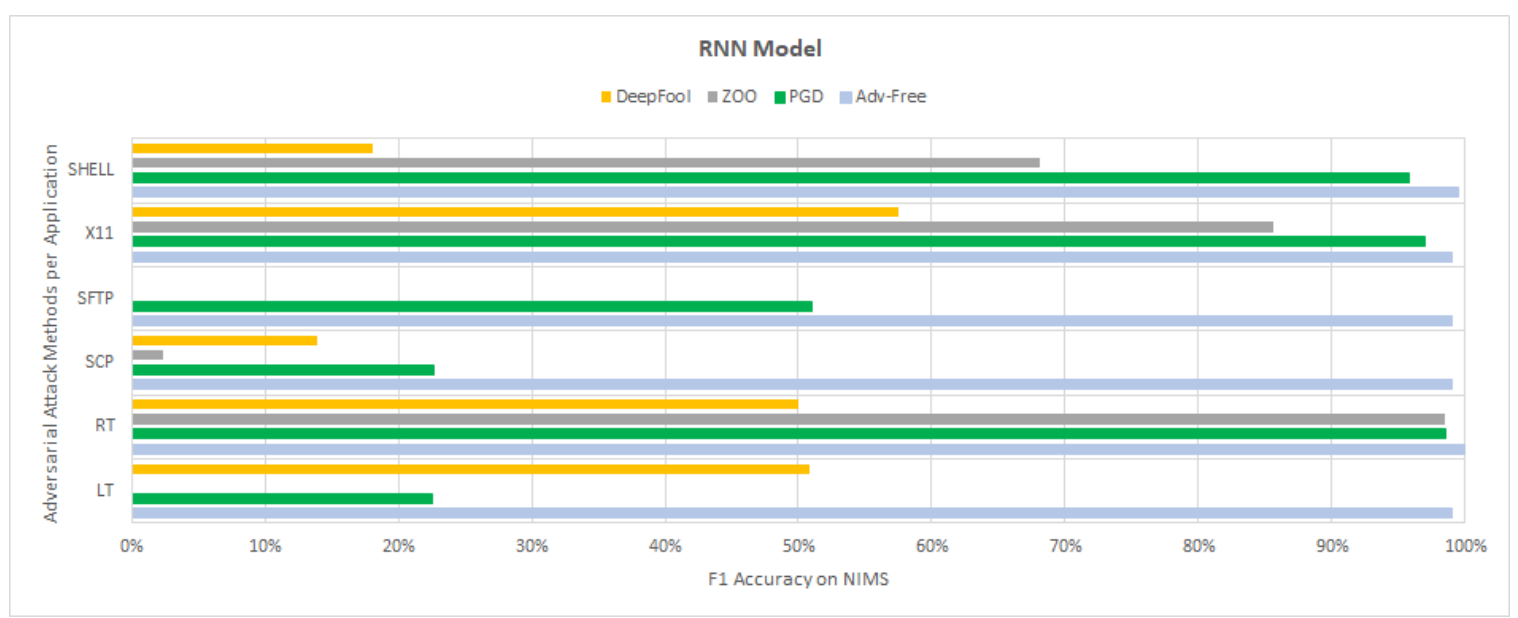

Figure 7.10: Effect of Adv. Samples generated on RNN models using Top FS trained by Adversarial-free NIMS dataset

Information. We evaluate and compare the resilience of the trained models in classifying encrypted traffic to that in Chapters 5 and 6 against the same adversarial attacks. From Figure 7.11 and 7.12, we can observe that both ML and DL model's accuracy in the adversarial-free environment and their resilience in classifying encrypted traffic against the generated adversarial samples were improved compared in Chapters 5 and 6.

In the ISCX VPN-NonVPN dataset in Figure 7.11, we can conclude that, on average, deep learning models were more resilient in classifying encrypted traffic to ZOO and DeepFool attacks compared to machine learning models. We also find that the resilience of machine learning was lower against the PGD attack than deep learning. We can conclude that the PGD attack has the most impact on the ISCX VPN-NonVPN dataset.

In NIMS dataset, on average, deep learning performance shows more resilience in classifying encrypted traffic against ZOO and PGD adversarial samples, compared to machine learning, as shown in Figure 6.12. Unlike DeepFool attack, where machine 


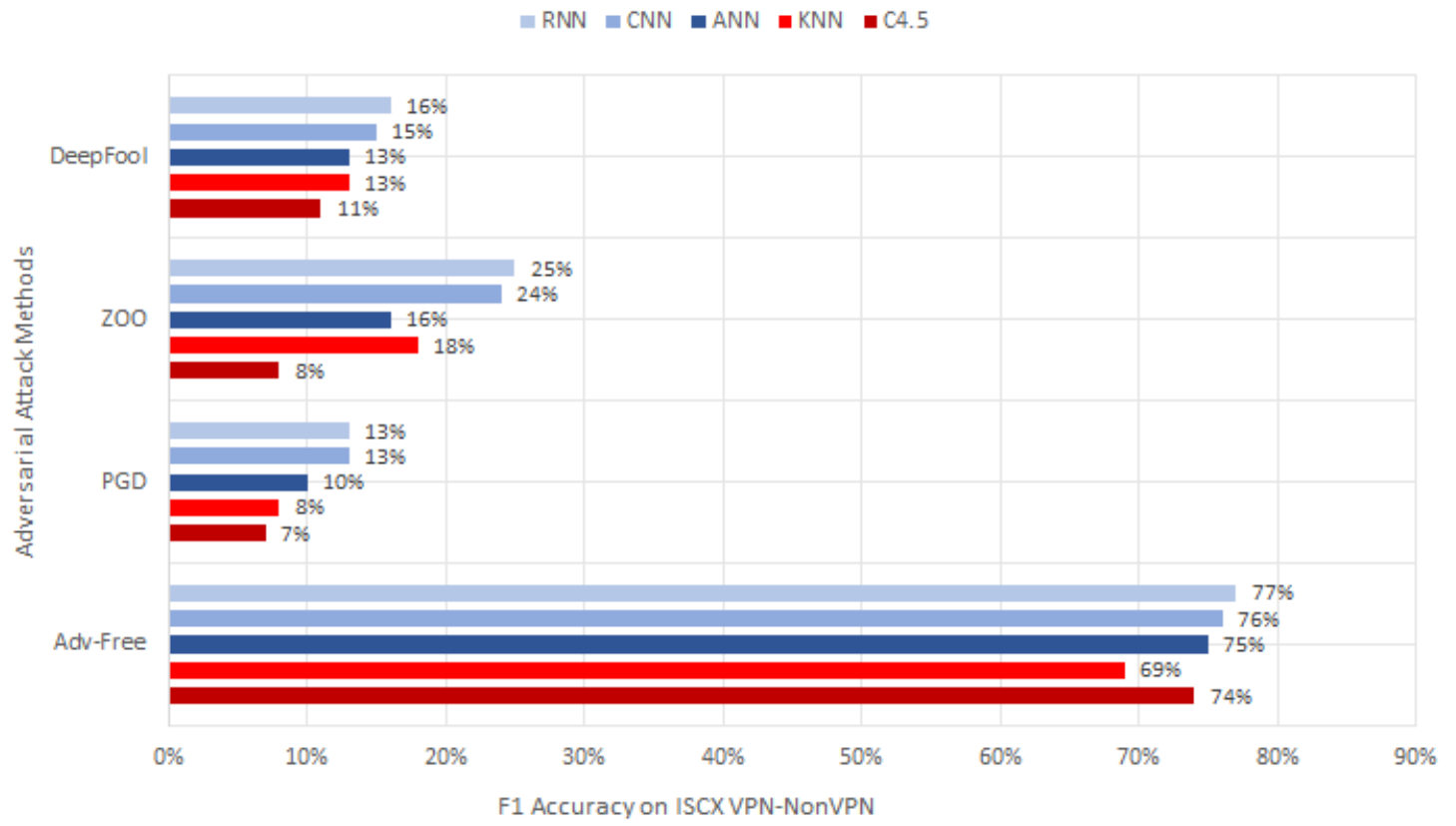

Figure 7.11: Effect of Adversarial Samples generated on five models using Top FS trained by Adversarial-free ISCX VPN-NonVPN dataset. DL algorithms are shown in different shades of Blue. ML algorithms are shown in different shades of Red.

learning was less resilient to this type of attack compared to deep learning. We can also conclude that the DeepFool attack method has the highest effect on NIMS dataset. 


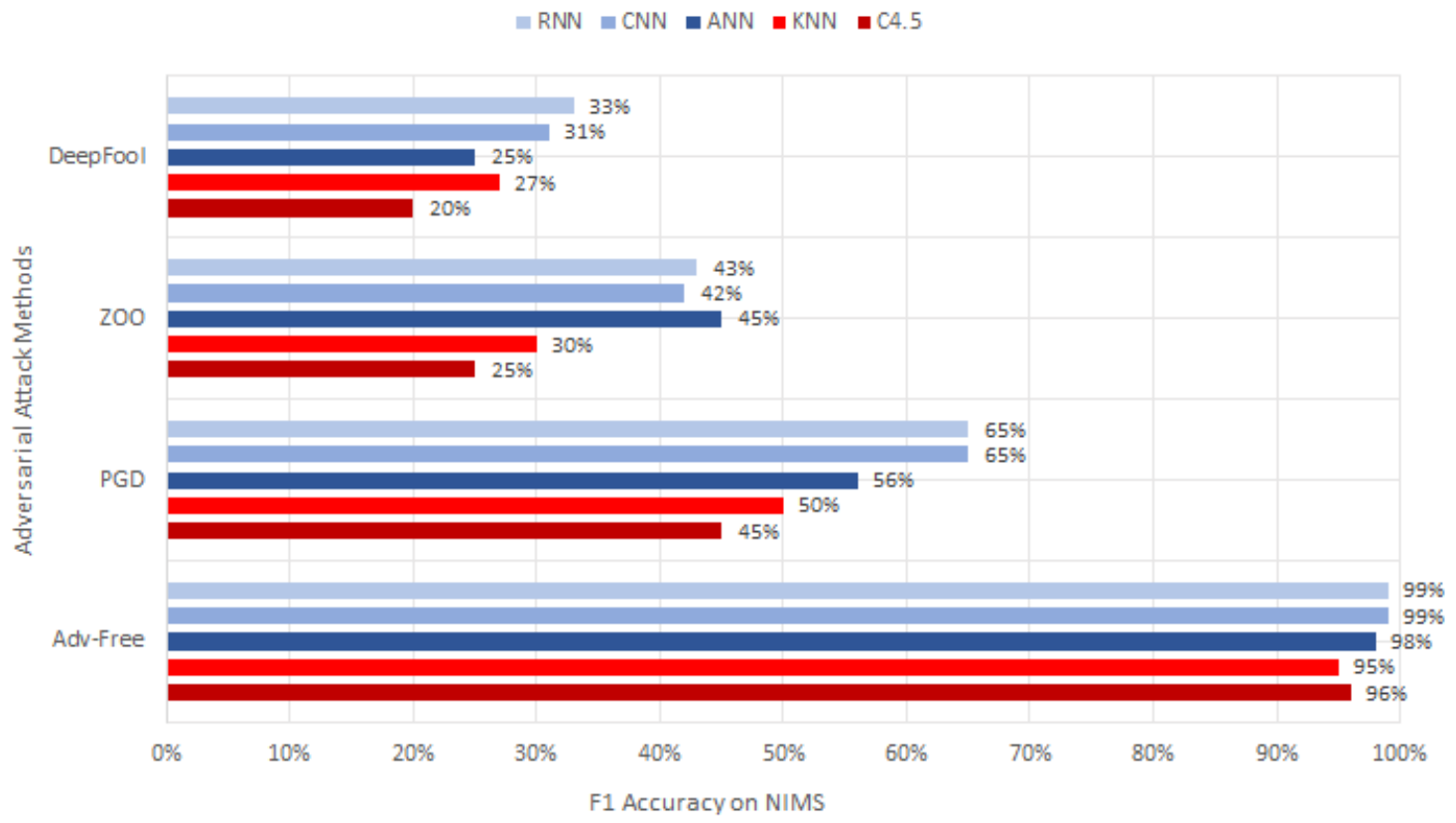

Figure 7.12: Effect of Adversarial Samples generated on five models using Top FS trained by Adversarial-free NIMS dataset. DL algorithms are shown in different shades of Blue. ML algorithms are shown in different shades of Red. 


\section{Chapter 8}

\section{Conclusions}

\subsection{Summary}

In this research, we attempt to study the resilience of trained models in classifying encrypted traffic against adversarial perturbations techniques in an adversarial environment. The Novelty of this research derives from the fact that it is the first experiment that implements and compares machine learning, e.g., C4.5 and KNN, and deep learning, e.g., ANN, CNN, and RNN, architectures in encrypted traffic classifications in an adversarial environment, and evaluated on two benchmark datasets: ISCX VPN-NonVPN and NIMS using various adversarial attack methods. Most of the literature in the adversarial domain demonstrated the concept of using either machine or deep learning architecture [73] with different adversarial attacks [74] [38].

Sufficient research was also provided that confirms the high accuracy and fast processing speed of our chosen classifiers in classifying network traffic applications [57] [75]. One of the reasons we chose those datasets is that we were looking for encrypted datasets that are publicly available, and used for classification of different types of encrypted applications, as it contains encrypted traffic data collected from normal encryption traffic (i.e., HTTPS, SSL, SFTP), VPN sessions, and SSH sessions. 
This research took the path further in investigating and evaluating the resilience of different types of ML and DL models against three state-of-the-art adversarial attack methods: PGD, ZOO, and DeepFool. Part of the experiments that we conducted was to study and analyze the effect of feature selection on two public benchmark datasets: ISCX VPN-NonVPN and NIMS. We applied feature selection using the Mutual Information technique that is used for multilabel classification. We moved forward with the entire experiments using this technique by selecting the same top five features in Chapters 5 and 6 and selecting the top nine features per each dataset in Chapter 7.

Finally, we can summarize the results and findings in this work.

1. On average, the performance of deep learning models was better than machine learning models in an adversarial-free environment in terms of encrypted traffic classification on both datasets: ISCX VPN-NonVPN and NIMS, using the same top five features.

2. Our results in adversarial attack environment demonstrate that:

- On average, deep learning models show better resilience in classifying encrypted traffic against the adversarial samples in comparison to machine learning.

- DeepFool Attack has more impact on almost all the models in both datasets.

- PGD and ZOO attack effectiveness in ISCX VPN-NonVPN and NIMS experiments in most of the models are nearly similar, and the difference is minimal and difficult to observe. 
3. When we retrained the models using the top nine selected features, our results demonstrate that:

- On average, all the model's accuracy in classifying encrypted traffic in an adversarial-free environment and their resilience against adversarial attacks were better than the results obtained in the initial training using the same top features.

- On average, deep learning models show better resilience against the adversarial samples in comparison to machine learning, using the top nine features.

- PGD Attack has more influence on almost all the models in ISCX VPNNonVPN, unlike NIMS, where DeepFool was more effective than the other attacks.

- We can conclude that as more top selected features are applied, the model's performance is enhanced in both adversarial-free and adversarial attack environments.

\subsection{Future Work}

Although ML and DL models showed some resilience against state-of-art adversarial attacks in classifying one level of encrypted traffic applications (VPN or SSH), we believe the exploration of classifying two encrypted traffic levels, i.e., one level encryption from HTTPS or SSL, and using VPN on top of it as the second encryption level, might leads to optimal learning model's resilience in an adversarial environment. 


\section{Bibliography}

[1] G. Draper-Gil, A. H. Lashkari, M. S. I. Mamun, and A. A. Ghorbani, "Characterization of encrypted and vpn traffic using time-related," in Proceedings of the 2nd international conference on information systems security and privacy (ICISSP), pp. 407-414, 2016.

[2] R. Alshammari and A. N. Zincir-Heywood, "Can encrypted traffic be identified without port numbers, ip addresses and payload inspection?," Computer networks, vol. 55, no. 6, pp. 1326-1350, 2011.

[3] R. Alshammari and A. N. Zincir-Heywood, "Investigating two different approaches for encrypted traffic classification," in 2008 Sixth Annual Conference on Privacy, Security and Trust, pp. 156-166, IEEE, 2008.

[4] R. Alshammari and A. N. Zincir-Heywood, "A flow based approach for ssh traffic detection," in 2007 IEEE international conference on systems, man and cybernetics, pp. 296-301, IEEE, 2007.

[5] A. Huet, A. Saverimoutou, Z. B. Houidi, H. Shi, S. Cai, J. Xu, B. Mathieu, and D. Rossi, "Revealing qoe of web users from encrypted network traffic," in 2020 IFIP Networking Conference (Networking), pp. 28-36, IEEE, 2020. 
[6] R. Sommer and V. Paxson, "Outside the closed world: On using machine learning for network intrusion detection," in 2010 IEEE symposium on security and privacy, pp. 305-316, IEEE, 2010.

[7] C. Szegedy, W. Zaremba, I. Sutskever, J. Bruna, D. Erhan, I. Goodfellow, and R. Fergus, "Intriguing properties of neural networks," arXiv preprint arXiv:1312.6199, 2013.

[8] S. Gu and L. Rigazio, "Towards deep neural network architectures robust to adversarial examples," arXiv preprint arXiv:1412.5068, 2014.

[9] Z. Wang, "Deep learning-based intrusion detection with adversaries," IEEE Access, vol. 6, pp. 38367-38384, 2018.

[10] I. Goodfellow, J. Shlens, and C. Szegedy, "Explaining and harnessing adversarial examples," in International Conference on Learning Representations, 2015.

[11] A. Kurakin, I. Goodfellow, and S. Bengio, "Adversarial examples in the physical world," ICLR Workshop, 2017.

[12] X. Yuan, P. He, Q. Zhu, and X. Li, "Adversarial examples: Attacks and defenses for deep learning," IEEE transactions on neural networks and learning systems, vol. 30, no. 9, pp. 2805-2824, 2019.

[13] S.-M. Moosavi-Dezfooli, A. Fawzi, and P. Frossard, "Deepfool: a simple and accurate method to fool deep neural networks," in Proceedings of the IEEE conference on computer vision and pattern recognition, pp. 2574-2582, 2016.

[14] U. Jang, X. Wu, and S. Jha, "Objective metrics and gradient descent algorithms for adversarial examples in machine learning," in Proceedings of the 33rd Annual Computer Security Applications Conference, pp. 262-277, 2017. 
[15] N. Papernot, P. McDaniel, S. Jha, M. Fredrikson, Z. B. Celik, and A. Swami, "The limitations of deep learning in adversarial settings," in 2016 IEEE European symposium on security and privacy (EuroS\&P), pp. 372-387, IEEE, 2016.

[16] A. Madry, A. Makelov, L. Schmidt, D. Tsipras, and A. Vladu, "Towards deep learning models resistant to adversarial attacks," in International Conference on Learning Representations, 2018.

[17] P.-Y. Chen, H. Zhang, Y. Sharma, J. Yi, and C.-J. Hsieh, "Zoo: Zeroth order optimization based black-box attacks to deep neural networks without training substitute models," in Proceedings of the 10th ACM workshop on artificial intelligence and security, pp. 15-26, 2017.

[18] R. Maarouf, D. Sattar, and A. Matrawy, "Evaluating resilience of encrypted traffic classification against adversarial evasion attacks," arXiv preprint arXiv:2105.14564, 2021.

[19] T. Rid and B. Buchanan, "Attributing cyber attacks," Journal of Strategic Studies, vol. 38, no. 1-2, pp. 4-37, 2015.

[20] M. Uma and G. Padmavathi, "A survey on various cyber attacks and their classification.," Int. J. Netw. Secur., vol. 15, no. 5, pp. 390-396, 2013.

[21] C. LaBounty, "What is network security and why is it important?."

[22] A. Singh, N. Thakur, and A. Sharma, "A review of supervised machine learning algorithms," in 2016 3rd International Conference on Computing for Sustainable Global Development (INDIACom), pp. 1310-1315, Ieee, 2016. 
[23] G. L. Agrawal and H. Gupta, "Optimization of c4. 5 decision tree algorithm for data mining application," International Journal of Emerging Technology and Advanced Engineering, vol. 3, no. 3, pp. 341-345, 2013.

[24] J. R. Quinlan, C4. 5: programs for machine learning. Elsevier, 2014.

[25] X. Wu, V. Kumar, J. R. Quinlan, J. Ghosh, Q. Yang, H. Motoda, G. J. McLachlan, A. Ng, B. Liu, S. Y. Philip, et al., "Top 10 algorithms in data mining," Knowledge and information systems, vol. 14, no. 1, pp. 1-37, 2008.

[26] J. M. Keller, M. R. Gray, and J. A. Givens, "A fuzzy k-nearest neighbor algorithm," IEEE transactions on systems, man, and cybernetics, no. 4, pp. 580-585, 1985

[27] K. Gayathri and A. Marimuthu, "Text document pre-processing with the knn for classification using the svm," in 2013 7th International Conference on Intelligent Systems and Control (ISCO), pp. 453-457, IEEE, 2013.

[28] S. Dargan, M. Kumar, M. R. Ayyagari, and G. Kumar, "A survey of deep learning and its applications: A new paradigm to machine learning," Archives of Computational Methods in Engineering, pp. 1-22, 2019.

[29] R. Vinayakumar, K. Soman, and P. Poornachandran, "Applying deep learning approaches for network traffic prediction," in 2017 International Conference on Advances in Computing, Communications and Informatics (ICACCI), pp. 23532358, IEEE, 2017.

[30] R. E. Uhrig, "Introduction to artificial neural networks," in Proceedings of IECON'95-21st Annual Conference on IEEE Industrial Electronics, vol. 1, pp. 33-37, IEEE, 1995. 
[31] S. Sharma, "Activation functions in neural networks," towards data science, vol. 6, 2017.

[32] X. Glorot, A. Bordes, and Y. Bengio, "Deep sparse rectifier neural networks," in Proceedings of the fourteenth international conference on artificial intelligence and statistics, pp. 315-323, JMLR Workshop and Conference Proceedings, 2011.

[33] H. U. Dike, Y. Zhou, K. K. Deveerasetty, and Q. Wu, "Unsupervised learning based on artificial neural network: A review," in 2018 IEEE International Conference on Cyborg and Bionic Systems (CBS), pp. 322-327, IEEE, 2018.

[34] A. Khan, A. Sohail, U. Zahoora, and A. S. Qureshi, "A survey of the recent architectures of deep convolutional neural networks," Artificial Intelligence Review, vol. 53, no. 8, pp. 5455-5516, 2020.

[35] W. Wang, M. Zhu, J. Wang, X. Zeng, and Z. Yang, "End-to-end encrypted traffic classification with one-dimensional convolution neural networks," in 2017 IEEE International Conference on Intelligence and Security Informatics (ISI), pp. 43-48, IEEE, 2017.

[36] S. Hochreiter and J. Schmidhuber, "Long short-term memory," Neural computation, vol. 9, no. 8, pp. 1735-1780, 1997.

[37] P. Laskov and R. Lippmann, "Machine learning in adversarial environments," 2010.

[38] J. Li, W. Monroe, T. Shi, S. Jean, A. Ritter, and D. Jurafsky, "Adversarial learning for neural dialogue generation," in Proceedings of the 2017 Conference on Empirical Methods in Natural Language Processing, (Copenhagen, Denmark), pp. 2157-2169, Association for Computational Linguistics, Sept. 2017. 
[39] P. Russu, A. Demontis, B. Biggio, G. Fumera, and F. Roli, "Secure kernel machines against evasion attacks," in Proceedings of the 2016 ACM workshop on artificial intelligence and security, pp. 59-69, 2016.

[40] V. Zantedeschi, M.-I. Nicolae, and A. Rawat, "Efficient defenses against adversarial attacks," in Proceedings of the 10th ACM Workshop on Artificial Intelligence and Security, pp. 39-49, 2017.

[41] O. Ibitoye, R. Abou-Khamis, A. Matrawy, and M. O. Shafiq, "The threat of adversarial attacks on machine learning in network security-a survey," arXiv preprint arXiv:1911.02621, 2019.

[42] N. Akhtar and A. Mian, "Threat of adversarial attacks on deep learning in computer vision: A survey," Ieee Access, vol. 6, pp. 14410-14430, 2018.

[43] A. Chakraborty, M. Alam, V. Dey, A. Chattopadhyay, and D. Mukhopadhyay, "Adversarial attacks and defences: A survey," arXiv preprint arXiv:1810.00069, 2018.

[44] N. Papernot, P. McDaniel, X. Wu, S. Jha, and A. Swami, "Distillation as a defense to adversarial perturbations against deep neural networks," in 2016 IEEE symposium on security and privacy (SP), pp. 582-597, IEEE, 2016.

[45] F. Zhang, P. P. Chan, B. Biggio, D. S. Yeung, and F. Roli, "Adversarial feature selection against evasion attacks," IEEE transactions on cybernetics, vol. 46, no. 3, pp. 766-777, 2015.

[46] N. Pitropakis, E. Panaousis, T. Giannetsos, E. Anastasiadis, and G. Loukas, "A taxonomy and survey of attacks against machine learning," Computer Science Review, vol. 34, p. 100199, 2019. 
[47] B. Biggio, B. Nelson, and P. Laskov, "Poisoning attacks against support vector machines," in Proceedings of the 29th International Coference on International Conference on Machine Learning, ICML'12, (Madison, WI, USA), p. 1467-1474, Omnipress, 2012.

[48] W. Wei, L. Liu, M. Loper, S. Truex, L. Yu, M. E. Gursoy, and Y. Wu, "Adversarial examples in deep learning: Characterization and divergence," arXiv preprint arXiv:1807.00051, 2018.

[49] S. Chen, M. Xue, L. Fan, S. Hao, L. Xu, H. Zhu, and B. Li, "Automated poisoning attacks and defenses in malware detection systems: An adversarial machine learning approach," computers $\&$ security, vol. 73, pp. 326-344, 2018.

[50] B. Wu, S. Wang, X. Yuan, C. Wang, C. Rudolph, and X. Yang, "Towards defeating misclassification attacks against transfer learning," arXiv preprint arXiv:1908.11230, 2019.

[51] T. T. Nguyen and G. Armitage, "A survey of techniques for internet traffic classification using machine learning," IEEE communications surveys $\&$ tutorials, vol. 10 , no. 4, pp. 56-76, 2008.

[52] A. McGregor, M. Hall, P. Lorier, and J. Brunskill, "Flow clustering using machine learning techniques," in International workshop on passive and active network measurement, pp. 205-214, Springer, 2004.

[53] J. Erman, A. Mahanti, M. Arlitt, and C. Williamson, "Identifying and discriminating between web and peer-to-peer traffic in the network core," in Proceedings of the 16th international conference on World Wide Web, pp. 883-892, 2007. 
[54] P. Haffner, S. Sen, O. Spatscheck, and D. Wang, "Acas: automated construction of application signatures," in Proceedings of the 2005 ACM SIGCOMM workshop on Mining network data, pp. 197-202, 2005.

[55] A. W. Moore and D. Zuev, "Internet traffic classification using bayesian analysis techniques," in Proceedings of the 2005 ACM SIGMETRICS international conference on Measurement and modeling of computer systems, pp. 50-60, 2005.

[56] M. Abbasi, A. Shahraki, and A. Taherkordi, "Deep learning for network traffic monitoring and analysis (ntma): A survey," Computer Communications, 2021.

[57] M. Lotfollahi, M. J. Siavoshani, R. S. H. Zade, and M. Saberian, "Deep packet: A novel approach for encrypted traffic classification using deep learning," Soft Computing, vol. 24, no. 3, pp. 1999-2012, 2020.

[58] A. S. Iliyasu and H. Deng, "Semi-supervised encrypted traffic classification with deep convolutional generative adversarial networks," IEEE Access, vol. 8, pp. 118-126, 2019.

[59] M. Z. Alom, V. Bontupalli, and T. M. Taha, "Intrusion detection using deep belief networks," in 2015 National Aerospace and Electronics Conference (NAECON), pp. 339-344, IEEE, 2015.

[60] H. Xu, Y. Ma, H.-C. Liu, D. Deb, H. Liu, J.-L. Tang, and A. K. Jain, "Adversarial attacks and defenses in images, graphs and text: A review," International Journal of Automation and Computing, vol. 17, no. 2, pp. 151-178, 2020.

[61] I. Corona, G. Giacinto, and F. Roli, "Adversarial attacks against intrusion detection systems: Taxonomy, solutions and open issues," Information Sciences, vol. 239, pp. 201-225, 2013. 
[62] A. Dainotti, A. Pescape, and K. C. Claffy, "Issues and future directions in traffic classification," IEEE network, vol. 26, no. 1, pp. 35-40, 2012.

[63] J. Brownlee, Deep learning with Python: develop deep learning models on Theano and TensorFlow using Keras. Machine Learning Mastery, 2016.

[64] P. Raybaut, "Spyder-documentation," Available online at: pythonhosted. org, 2009.

[65] M.-I. Nicolae, M. Sinn, T. N. Minh, A. Rawat, M. Wistuba, V. Zantedeschi, I. M. Molloy, and B. Edwards, "Adversarial robustness toolbox v0. 2.2," 2018.

[66] J. Brownlee, "Machine learning mastery with python," Machine Learning Mastery Pty Ltd, vol. 527, pp. 100-120, 2016.

[67] J. Brownlee, "How to index, slice and reshape numpy arrays for machine learning."

[68] G. Chandrashekar and F. Sahin, "A survey on feature selection methods," Computers 8 Electrical Engineering, vol. 40, no. 1, pp. 16-28, 2014.

[69] A. Kraskov, H. Stögbauer, and P. Grassberger, "Estimating mutual information," Physical review E, vol. 69, no. 6, p. 066138, 2004.

[70] J. R. Vergara and P. A. Estévez, "A review of feature selection methods based on mutual information," Neural computing and applications, vol. 24, no. 1, pp. 175$186,2014$.

[71] H. Sharma and S. Kumar, "A survey on decision tree algorithms of classification in data mining," International Journal of Science and Research (IJSR), vol. 5, no. 4, pp. 2094-2097, 2016. 
[72] L. Jiang, Z. Cai, D. Wang, and S. Jiang, "Survey of improving k-nearest-neighbor for classification," in Fourth international conference on fuzzy systems and knowledge discovery (FSKD 2007), vol. 1, pp. 679-683, IEEE, 2007.

[73] B. Biggio, I. Corona, G. Fumera, G. Giacinto, and F. Roli, "Bagging classifiers for fighting poisoning attacks in adversarial classification tasks," in International workshop on multiple classifier systems, pp. 350-359, Springer, 2011.

[74] R. Abou Khamis and A. Matrawy, "Evaluation of adversarial training on different types of neural networks in deep learning-based idss," in 2020 International Symposium on Networks, Computers and Communications (ISNCC), pp. 1-6, IEEE, 2020.

[75] M. Shafiq, X. Yu, A. A. Laghari, L. Yao, N. K. Karn, and F. Abdessamia, "Network traffic classification techniques and comparative analysis using machine learning algorithms," in 2016 2nd IEEE International Conference on Computer and Communications (ICCC), pp. 2451-2455, IEEE, 2016. 
Appendix A 
Table A.1: ISCX VPN-NonVPN dataset Features based on the description in [1]

\begin{tabular}{||l|l|l||}
\hline ID & Name & Description \\
\hline \hline 1 & duration & Record total duration \\
\hline 2 & total-fiat & Total Forward Inter Arrival Time \\
\hline 3 & total-biat & Total Backward Inter Arrival Time \\
\hline 4 & min-fiat & Minumim Forward Inter Arrival Time \\
\hline 5 & min-biat & Minumim Backward Inter Arrival Time \\
\hline 6 & max-fiat & Maxumim Forward Inter Arrival Time \\
\hline 7 & max-biat & Maxumim Backward Inter Arrival Time \\
\hline 8 & mean-fiat & Mean Forward Inter Arrival Time \\
\hline 9 & mean-biat & Mean Backward Inter Arrival Time \\
\hline 10 & flowPktsPerSecond & Flow packets per second \\
\hline 11 & flowBytesPerSecond & Flow Bytes per second \\
\hline 12 & min-flowiat & Minumim Flow Inter Arrival Time \\
\hline 13 & max-flowiat & Maxumim Flow Inter Arrival Time \\
\hline 14 & mean-flowiat & Mean Flow Inter Arrival Time \\
\hline 15 & std-flowiat & Standard Deviation of Flow Inter Arrival Time \\
\hline 16 & min-active & Minumim amount of time a flow was active \\
\hline 17 & mean-active & Mean amount of time a flow was active \\
\hline 18 & max-active & Maxumim amount of time a flow was active \\
\hline 19 & std-active & Standard Deviation of the amount of time a flow was active \\
\hline 20 & min-idle & Minumim amount of time a flow was idle \\
\hline 21 & mean-idle & Mean amount of time a flow was idle \\
\hline 22 & max-idle & Maxumim amount of time a flow was idle \\
\hline 23 & std-idle & Standard Deviation of the amount of time a flow was idle \\
\hline
\end{tabular}


Table A.2: NIMS dataset Features based on the description in [2] [3] [4]

\begin{tabular}{||l|l|l||}
\hline ID & Name & Description \\
\hline \hline 1 & min-fpktl & Minumim forward packet length \\
\hline 2 & mean-fpktl & Mean forward packet length \\
\hline 3 & max-fpktl & Maximum forward packet length \\
\hline 4 & std-fpktl & Standard Deviation of forward packet length \\
\hline 5 & min-bpktl & Minumim backward packet length \\
\hline 6 & mean-bpktl & Mean backward packet length \\
\hline 7 & max-bpktl & Maximum backward packet length \\
\hline 8 & std-bpktl & Standard Deviation of backward packet length \\
\hline 9 & min-fiat & Minumim forward Inter Arrival Time \\
\hline 10 & mean-fiat & Mean forward Inter Arrival Time \\
\hline 11 & max-fiat & Maxumim forward Inter Arrival Time \\
\hline 12 & std-fiat & Standard Deviation of forward Inter Arrival Time \\
\hline 13 & min-biat & Minumim backward Inter Arrival Time \\
\hline 14 & mean-biat & Mean backward Inter Arrival Time \\
\hline 15 & max-biat & Maxumim backward Inter Arrival Time \\
\hline 16 & std-biat & Standard Deviation of backward Inter Arrival Time \\
\hline 17 & duration & Record total duration \\
\hline 18 & proto & Protocol \\
\hline 19 & total-fpackets & Total Packets in forward direction \\
\hline 20 & total-fvolume & Total Bytes in forward direction \\
\hline 21 & total-bpackets & Total Packets in backward direction \\
\hline 22 & total-bvolume & Total Bytes in backward direction \\
\hline
\end{tabular}

